$$
\begin{gathered}
\text { Universidade de São Paulo } \\
\text { Instituto de Física de São Carlos }
\end{gathered}
$$

André de Freitas Smaira

\title{
Dinâmica de um condensado de Bose-Einstein contendo sólitons
}





\section{André de Freitas Smaira}

\section{Dinâmica de um condensado de Bose-Einstein contendo sólitons}

Dissertação apresentada ao Programa de PósGraduação em Física do Instituto de Física de São Carlos da Universidade de São Paulo, para obtenção do título de Mestre em Ciências.

Área de Concentração: Física Aplicada

Opção: Física Computacional

Orientador: Prof. Dr. Vanderlei Salvador Bagnato

Versão Corrigida

(Versão original disponível na Unidade que aloja o Programa) 
AUTORIZO A REPRODUÇÃO E DIVULGAÇÃO TOTAL OU PARCIAL DESTE TRABALHO, POR QUALQUER MEIO CONVENCIONAL OU ELETRÔNICO, PARA FINS DE ESTUDO E PESQUISA, DESDE QUE CITADA A FONTE.

Ficha catalográfica elaborada pelo Serviço de Biblioteca e Informação do IFSC, com os dados fornecidos pelo(a) autor(a)

Smaira, André de Freitas

Dinâmica de um condensado de Bose-Einstein

contendo sólitons / André de Freitas Smaira.

orientador Vanderlei Salvador Bagnato - versão

corrigida -- São Carlos, 2015.

$112 \mathrm{p}$.

Dissertação (Mestrado - Programa de Pós-Graduação em Física Aplicada Computacional) - - Instituto de Física de São Carlos, Universidade de São Paulo, 2015.

1. Condensado de Bose-Einstein. 2. Matéria condensada. 3. Átomos ultra-frios. I. Bagnato, Vanderlei Salvador, orient. II. Título. 


\section{AGRADECIMENTOS}

Agradeço primeiramente aos meus pais (Luiz e Marta) e avós (Zeza e Ranulfo, Odette e Waldemar) pela melhor educação que eu poderia ter, tanto moral quanto escolar, sem os quais eu não teria tido o sucesso acadêmico que tive até o momento, e pela presença por toda minha vida até agora. Em seguida, agradeço aos meus três irmãos (Lucas, Clara e Nina) pelas conversas e discussões, em especial ao Lucas pelas conversas físicas, matemáticas e computacionais e pela ajuda nessa dissertação.

Agradeço aos meus primos (Gabriel, Dudu, Fernando e Rafael) e, novamente aos meus irmãos mais velhos (Lucas e Clara), por uma infância cheia de aprendizado com trotes e brincadeiras, de que tenho maravilhosas lembranças. Agradeço também aos meus tios (Tia Cecília e Tio Bené, Tia Ana, Tia Ângela e Tio Edu, Tia Ruthinha e Tio Rubener, Tia Anita e Tio João) pelo apoio, em especial ao Tio Rubener pelo incentivo nas Exatas, inclusive me fornecendo meu primeiro software matemático (MapleSoft 5.1), que me impulsionou em muitas descobertas.

Agradeço à minha namorada (Thereza) pelo apoio tanto pessoal quanto acadêmico (MatLab, em especial), sem o qual provavelmente eu não estaria defendendo o mestrado nesse momento.

Agradeço também às amizades que surgiram durante a Graduação: Thereza (e Marta), Cabeça (José Teixeira), Chernobil (Danilo), PdPano (Vinicius), Paulo Matias, Krissia, Velma (Jessica), Diogo, entre outros, com dias e noites de estudos e competições extremamente exaustivas; e durante o Mestrado: Rafael, Mônica, André, Lhaís entre outros, com discussões científicas e fins de semana de descanso, e que permanecem até hoje, sem as quais minha vida acadêmica não seria a mesma.

Agradeço aos integrantes do GEMA, Grupo de Estudo para a MAratona de Programação, do ICMC: Prof. Gustavo, RG (Rafael), PH (Raphael), Quake (Vinicius), Rafael Regis, Diego, Pirata (Filipe), Bianca, Denis, Bruno Adami, Lucas, Bruno Sanches, Shi (Luís Dorelli), entre outros, pela amizade e pelo enorme carga de conhecimento de programação e algoritmos obtidas nas reuniões semanais do Grupo e nas competições de programação.

Agradeço a todos meus Professores (vou citar apenas os que mais me marcaram positivamente pois são dezenas, ou talvez centenas), seja do Ensino Fundamental (Dona Mariinha, Ana Lúcia Bárbara Cruvinel, Adriana Carvalho dos Santos, Léa Cristina de Carvalho Pedrosa, Lourdes Helena Borelli Zeller, Luiz Antonio Pasotti Smaira - Pai, Tia Regina), do Ensino Mé- 
dio (Ana Cláudia Pinto Corrêa, Flávia Ribeiro do Valle Gonçalves, Mário Pasqua Filho, Valdir Veronezi, Rubão, Renato Gomes de Carvalho, Elias Avancini de Brito, Sergio Luis Ferro, Alex Sander Schroeder de Barros, Rodolfo de Santis Neves, Daniel Pereira Leite Palenewen, Emerson de Maria, Umberto César Chacon Malanga, Renato Alberto Rodrigues, Guilherme Aulicino Bastos Jorge, Homero Galvão), da Graduação (Vanderlei Salvador Bagnato, Francisco Castilho Alcaraz, Tito José Bonagamba, Wagner Vieira Leite Nunes, Djalma Mirabelli Redondo, Gonzalo Travieso, Luiz Nunes de Oliveira, Luiz Agostinho Ferreira, Eduardo Ribeiro de Azevêdo, Attilio Cucchieri, Cleber Renato Mendonça, Lidério Citrângulo loriatti Júnior, Carlos Antônio Ruggiero - Totó, Reynaldo Daniel Pinto) ou da Pós-Graduação (Reginaldo de Jesus Napolitano, Gonzalo Travieso), tanto aos bons (em parte citados anteriormente), pelo aprendizado em todas as áreas, quanto aos ruins, pelas dicas do que não fazer caso venha a me tornar professor algum dia.

Não posso esquecer de agradecer também aos técnicos do laboratório de ensino (Antenor Fabbri Petrilli Filho, Cláudio Boense Bretas, Daniele Santini Jacinto, Ércio Santoni, Marcos José Semenzato - Marcão, entre outros), pelo muito que fizeram para nos ajudar quando mais precisávamos durante a Graduação.

Agradeço ao meu orientador (Vanderlei Salvador Bagnato), aos meus co-orientadores não oficiais, mas super importantes (Stella, Daniel e Mônica), aos integrantes do meu grupo e colaboradores (André, Rafael, Ednilson, Axel) pelas discussões e dicas inicialmente no TAC (Iniciação Científica) e depois no BEC (Iniciação Científica e Mestrado), que me trouxeram, entre muitas outras conquistas, mais esse título acadêmico.

Agradeço também às secretárias do Grupo de Óptica (Isabel, Benê e Cristiane) e aos membros das Secretarias de Graduação (Edvane e Isabel) e Pós-Graduação (Silvio, Patricia e Ricardo) do IFSC pela solução dos problemas burocráticos e pela paciência desde o início da minha Graduação.

Por último agradeço às agências financiadoras (CNPq e FAPESP) que me financiaram junto ao nosso grupo de pesquisa desde minha iniciação científica (iniciada no começo de 2010) até o presente momento, quando finalizo meu Mestrado. 
A gratidão é a memória do coração. 

Esta dissertação foi financiada pela FAPESP. 



\section{RESUMO}

SMAIRA, A. F. Dinâmica de um condensado de Bose-Einstein contendo sólitons. 2015. 112 p. Dissertação de Mestrado - Instituto de Física de São Carlos, Universidade de São Paulo, São Carlos, 2015.

Condensados de Bose-Einstein (BEC) são sistemas macroscópicos excelentes para a observação do comportamento quântico da matéria. Desde sua obtenção experimental em gases atômicos alcalinos diluídos aprisionados por campos magnéticos, há importantes aspectos relacionados a esse sistema que foram intensamente explorados, como os modos coletivos do BEC harmonicamente aprisionado, seu tunelamento através de barreiras de potencial e os estados excitados desse sistema, incluindo vórtice e sóliton. O último consiste de pacote de onda localizado, que propaga sem mudança de forma. Nesse trabalho, investigamos os novos aspectos que surgem da dinâmica de um sistema composto (condensado aprisionado contendo um sóliton). Há muitos estudos tratando cada parte separadamente: estado fundamental do BEC ou um sóliton em um BEC infinito uniforme estacionário. Estamos nos baseando nessas análises prévias, além da simulação numérica de campo médio do nosso sistema submetido a diferentes condições iniciais (BEC aprisionado no mínimo do potencial harmônico ou BEC deslocado na armadilha contendo um sóliton, além de uma deformação no potencial) para caracterizar a dinâmica desse sistema. Alguns dos nossos resultados puderam ser explicados por meio de predições analítica da chamada aproximação de Thomas-Fermi. Ao final, comparamos as simulações de campo médio (equação de Gross-Pitaevskii) com as advindas da teoria de múltiplos orbitais a fim de justificar o regime de validade da nossa teoria.

Palavras-chave: Condensado de Bose-Einstein. Matéria condensada. Átomos ultra-frios. 



\section{ABSTRACT}

SMAIRA, A. F. Bose-Einstein condensate dynamics with solitons. 2015. 112 p. Dissertação de Mestrado - Instituto de Física de São Carlos, Universidade de São Paulo, São Carlos, 2015.

Bose-Einstein Condensates (BEC) are excellent macroscopic systems to observe the quantum behavior of matter. Since it experimental production in dilute atomic alkali gases trapped by magnetic fields, there are important aspects related to this system that have been intensely explored, like the collective modes of the harmonically trapped BEC, its tunneling through a potential barrier and the excited states of this system, that include the vortex and soliton. The latter consist of localized disturbances, which propagate without change of form. In this work, we investigate the singular aspects that coming from the dynamics of a composite system (trapped BEC containing a soliton). There are many studies that treat each part separately, that include a fundamental state BEC or a soliton inside a uniform infinite extent stationary BEC. We are basing on these previous analyses, besides mean-field numeric simulating our particular system submitted to diferent initial conditions (minimum harmonic potential trapped $\mathrm{BEC}$ or dislocated trapped BEC plus a soliton, in addition to a deformation in the potential) to characterize the tunneling dynamics. Some of our results could be explained using analytical predictions of the so called Thomas-Fermi approximation. At the end, we compar the meanfield simulations (Gross-Pitavskii equation) with the simulations from the multiple orbitals theory to justify the validity regime of our theory.

Keywords: Bose-Einstein condensate. Matter wave soliton. Ultracold atoms. 



\section{LISTA DE FIGURAS}

Figura 1.1 - Distribuição estatística de partículas para três valores de $\mathcal{G}$

Figura 3.1 - Imagem de um movimento genérico de um BEC deslocado do centro da armadilha

Figura 3.2 - Evolução temporal do primeiro período $(T)$ da função de onda relativa à figura 3.1 .

Figura 3.3 - Modo dipolar para $\omega=1$.

Figura 3.4 - Modo dipolar para $\omega=2$.

Figura 3.5 - Modo dipolar para $\omega=3$.

Figura 3.6 - Condicão inicial do sistema BEC+grey soliton inicialmente centrado na armadilha

Figura 3.7 - Imagem do movimento do grey soliton em um BEC inicialmente centrado na armadilha

Figura 3.8 - Dinâmica do sóliton em um BEC inicialmente centrado na armadilha .

Figura 3.9 - Dinâmica do CM do sistema BEC+grey soliton inicialmente centrado na armadilha

Figura 3.10 -Dinâmica do CM do sistema BEC+black soliton inicialmente centrado na armadilha

Figura 3.11 -Condicão inicial do sistema BEC+grey soliton inicialmente deslocado do centro da armadilha .

Figura 3.12 -Imagem do movimento do sistema BEC+black soliton inicialmente deslocado do centro da armadilha

Figura 3.13 -Dinâmica de um black soliton em um BEC inicialmente deslocado do centro da armadilha

Figura 3.14 -Imagem do movimento do sistema BEC+grey soliton inicialmente deslocado do centro da armadilha 
Figura 3.15 -Condicão inicial do BEC inicialmente deslocado do centro da armadilha

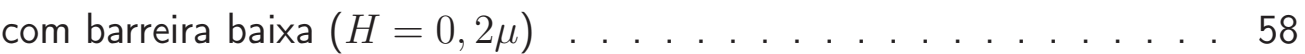

Figura 3.16 -Imagem do movimento de um BEC inicialmente deslocado do centro da armadilha com barreira baixa $(H=0,2 \mu) \ldots \ldots$. . . . . . . 58

Figura 3.17 -Dinâmica do BEC inicialmente deslocado do centro da armadilha com barreira baixa $(H=0,2 \mu) \ldots \ldots . \ldots . \ldots . . \ldots 59$

Figura 3.18 -Condicão inicial do BEC inicialmente deslocado do centro da armadilha

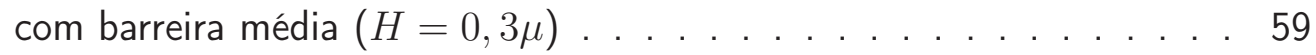

Figura 3.19 -Imagem do movimento do BEC inicialmente deslocado do centro da armadilha com barreira média $(H=0,3 \mu) \ldots 60$

Figura 3.20 -Dinâmica do BEC inicialmente deslocado do centro da armadilha com barreira média $(H=0,3 \mu) \ldots \ldots$. . . . . . . . . . . 60

Figura 3.21 -Condicão inicial do BEC inicialmente deslocado do centro da armadilha com barreira alta $(H=0,5 \mu) \ldots \ldots$. . . . . . . . . . 61

Figura 3.22 -Imagem do movimento de um BEC inicialmente deslocado do centro da armadilha com barreira alta $(H=0,5 \mu) \ldots . . \ldots 61$

Figura 3.23 -Dinâmica do BEC inicialmente deslocado do centro da armadilha com barreira alta $(H=0,5 \mu) \ldots \ldots . \ldots . \ldots 62$

Figura 3.24 -Condicão inicial do BEC inicialmente deslocado do centro da armadilha com barreira bem maior que a energia do $\operatorname{BEC}(H=5 \mu)$. . . . . . . 62

Figura 3.25 -Imagem do movimento do BEC inicialmente deslocado do centro da armadilha com barreira de altura $H=5 \mu \ldots \ldots$

Figura 3.26 -Dinâmica do $\mathrm{CM}$ e do raio do BEC, respectivamente, com $H=5 \mu$. . 63

Figura 3.27 -Condicões iniciais do sistema BEC+grev soliton inicialmente deslocado do centro da armadilha com barreiras de alturas $H \in\{0,1 \mu ; 0,2 \mu ; 0,3 \mu\} 64$

Figura 3.28 -Imagem do movimento de um grey soliton em um BEC inicialmente centrado na armadil ha com uma barreira de alturas $H \in\{0,1 \mu ; 0,2 \mu ; 0,3 \mu\}$, respectivamente . . . . . . . . . . . . . . . . 65

Figura 3.29 -Dinâmica do grev soliton em um BEC inicialmente centrado na armadilha com barreiras de alturas $H \in\{0,1 \mu ; 0,2 \mu ; 0,3 \mu\}$, respectivamente 65 
Figura 3.30 - Transformada de Fourier da dinâmica do grey soliton em um BEC inicialmente centrado na armadilha com barreiras de alturas $H \in\{0,1 \mu ; 0,2 \mu ; 0,3 \mu\}$, respectivamente ....................... 66

Figura 3.31 -Dinâmica do $\mathrm{CM}$ do sistema $\mathrm{BEC}+$ grey soliton inicialmente centrado na armadillha com barreiras de alturas $H \in\{0,1 \mu ; 0,2 \mu ; 0,3 \mu\}$, respectivamente . . . . . . . . . . . . . . . . 6 67

Figura 3.32 - Transformadas de Fourier da dinâmica do CM do sistema BEC+grey soliton inicialmente centrado na armadilha com barreiras de $H \in\{0,1 \mu ; 0,2 \mu ; 0,3 \mu\}$, respectivamente

Figura 3.33 -Condições iniciais do sistema BEC+black soliton inicialmente deslocado do centro da armadilha com barreiras de alturas $H=0,2 \mu$ e $H=0,3 \mu$, respectivamente

Figura 3.34 -Imagens do movimento do sistema BEC+black soliton inicialmente deslocado do centro da armadilha com barreiras de alturas $H=0,2 \mu \mathrm{e}$ $H=0,3 \mu$, respectivamente

Figura 3.35 -Dinâmica do black soliton inicial do sistema BEC+black soliton inicialmente deslocado do centro da armadilha com barreiras de $H \in$ $\{0,1 \mu ; 0,2 \mu ; 0,3 \mu\}$, respectivamente

Figura 3.36 -Transformada de Fourier dinâmica do black soliton inicial do sistema BEC+black soliton inicialmente deslocado do centro da armadilha com barreiras de $H \in\{0,1 \mu ; 0,2 \mu ; 0,3 \mu\}$, respectivamente . . . . . . . 70

Figura 3.37 -Dinâmica do CM do sistema BEC+black soliton inicialmente deslocado do centro da armadilha com barreiras de $H \in\{0,1 \mu ; 0,2 \mu ; 0,3 \mu\}$, respectivamente

Figura 3.38 -Condicão inicial do sistema BEC+black soliton inicialmente deslocado do centro da armadilha com barreira de altura $H=5 \mu \ldots \ldots$. . . . 71

Figura 3.39-Imagem do movimento do sistema BEC+black soliton inicialmente deslocado do centro da armadilha com uma barreira de altura $H=5 \mu$. .

Figura 3.40 - Transformada de Fourier da dinâmica do black soliton inicial do sistema BEC+sóliton inicialmente deslocado do centro da armadilha com

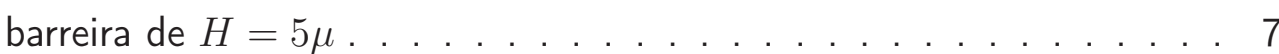


Figura 3.41 -Transformada de Fourier da dinâmica do CM e do raio do BEC, respectivamente, inicialmente deslocado do centro da armadilha . . . . . . 73

Figura 3.42 -Condicão inicial de um BEC inicialmente deslocado do centro da armadilha com rugosidade uniformemente distribuída . . . . . . . . . 74

Figura 3.43-Imagem do movimento do BEC inicialmente deslocado do centro da armadilha com rugosidade uniformemente distribuída . . . . . . 75

Figura 3.44 -Dinâmica do CM de um BEC inicialmente deslocado do centro de uma armadilha harmônica com rugosidade uniformemente distribuída . . . 75

Figura 3.45 -Dinâmica do raio de um BEC inicialmente deslocado do centro de uma armadilha harmônica com rugosidade uniformemente distribuída . . . 76

Figura 3.46 -Condicão inicial de um BEC inicialmente deslocado do centro da armadilha com rugosidade espcialmente não uniforme . . . . . . . . 77

Figura 3.47-Imagem do movimento do BEC inicialmente deslocado do centro da armadilha com rugosidade espcialmente não uniforme . . . . . . . . 77

Figura 3.48 -Dinâmica do CM de um BEC inicialmente deslocado do centro de uma armadilha harmônica com rugosidade espacialmente não uniforme . . . 78

Figura 3.49 -Dinâmica do raio de um BEC inicialmente deslocado do centro de uma armadilha harmônica com rugosidade espacialmente não uniforme . . . 78

Figura 3.50 -Condições iniciais do sistema BEC+sóliton inicialmente deslocado do centro da armadilha com rugosidade uniformemente distribuída, para $u=0$ e $u=0,5$, respectivamente . . . . . . . . . . . 79

Figura 3.51 -Imagens dos movimentos do BEC inicialmente deslocado do centro da armadilha com rugosidade uniformemente distribuída, para $u=0 \mathrm{e}$ $u=0,5$, respectivamente . . . . . . . . . . . . . 79

Figura 3.52 -Dinâmica do CM de um BEC inicialmente deslocado do centro de uma armadilha harmônica com rugosidade uniformemente distribuída, para $u=0$ e $u=0,5$, respectivamente

Figura 3.53 -Dinâmica do sóliton em um BEC inicialmente deslocado do centro de uma armadilha harmônica com rugosidade uniformemente distribuída, para $u=0$ e $u=0,5$, respectivamente 
Figura 3.54 -Dinâmica do raio de um BEC inicialmente deslocado do centro de uma armadilha harmônica com rugosidlade uniformemente distribuída, para $u=0$ e $u=0,5$, respectivamente . . . . . . . . . . . . . . 81

Figura 3.55 -Condições iniciais do sistema BEC+sóliton inicialmente deslocado do centro da armadilha com rugosidade espacialmente não uniforme, para $u=0$ e $u=0,5$, respectivamente . . . . . . . . . . . . . .

Figura 3.56 -Imagens dos movimentos do sistema BEC+sóliton inicialmente deslocado do centro na armadilha com rugosidade espacialmente não uniforme, para $u=0$ e $u=0,5$, respectivamente

Figura 3.57 -Dinâmica do CM de um sistema BEC+sóliton inicialmente deslocado do centro de uma armadilha harmônica com rugosidade espacialmente não uniforme, para $u=0$ e $u=0,5$, respectivamente

Figura 3.58 -Dinâmica do sóliton em um BEC inicialmente deslocado do centro de uma armadilha harmônica com rugosidade espacialmente não uniforme, para $u=0$ e $u=0,5$, respectivamente

Figura 3.59 -Dinâmica do raio de um BEC inicialmente deslocado do centro de uma armadilha harmônica com rugosidade espacialmente não uniforme, para $u=0$ e $u=0,5$, respectivamente

Figura 4.1 - Evolução da função de onda de um BEC+sóliton inicialmente centrado na armadilha (simulação da GPE) para $t \in\left\{0 ; \frac{T}{4} ; \frac{T}{2} ; \frac{3 T}{4} ; T\right\}$, respectivamente, sendo $T$ o período de oscilação do sóliton

Figura 4.2 - Evolução da função de onda de um BEC+sóliton inicialmente centrado na armadilha (simulação MCTDHB com $M=1$ ) para $t \in$ $\left\{0 ; \frac{T}{4} ; \frac{T}{2} ; \frac{3 T}{4} ; T\right\}$, respectivamente, sendo $T$ o período de oscilação

Figura 4.3 - Evolução da função de onda de um BEC+sóliton inicialmente centrado na armadilha (simulação MCTDHB com $M=2$ ) para $t \in\left\{0 ; \frac{T}{8} ; \frac{T}{4}\right\}$, respectivamente, sendo $T$ o período de oscilação do sóliton . . . . . . . . 



\section{LISTA DE TABELAS}

Tabela A.1 -Constantes Físicas . . . . . . . . . . . . . . . . . . . . . . . . . 95 



\section{LISTA DE ACRÔNIMOS}

1D - Unidimensional

BEC - Condensado de Bose-Einstein

CM - Centro de Massa

GPE - Equação de Gross-Pitaevskii

MOT - Armadilha Magneto-Óptica

RTF - Raio de Thomas-Fermi

TF - Thomas-Fermi

TFA - Aproximação de Thomas-Fermi 



\section{SUMÁRIO}

1 Introdução

$1.1 \quad$ Condensado de Bose-Einstein . . . . . . . . . . . . . . . 25

1.1.1 Gás de bósons não interagentes aprisionados . . . . . . . . . . . . . . . 26

1.1.1.1 Fração condensada . . . . . . . . . . . . . . . . . . . . . . . . . . . . . . . . . 29

1.1 .2 Equação de Gross-Pitaevskii (GPE) . . . . . . . . . . . . . . . . . . . . . . . . . . . 29

1.1.2.1 Aproximação de Thomas-Fermi (TFA) . . . . . . . . . . . . . . . . . . . . 33

1.1.2.2 Equação dependente do tempo . . . . . . . . . . . . . . . . . . . 34

1.1.2.3 Modos coletivos . . . . . . . . . . . . . . . . . . . . . 34

1.1 .3 Sistema unidimensional . . . . . . . . . . . . . . . . . . . . . 35

$1.2 \quad$ Estados Excitados . . . . . . . . . . . . . . . . . . . . . . . 38

1.2 .1 Sólitons . . . . . . . . . . . . . . . . . . . . . . . . . 38

1.2 .2 Sólitons em BECs aprisionados . . . . . . . . . . . . . . . . . . . . . . . . 40

1.3 Teoria de Múltiplos Orbitais . . . . . . . . . . . . . . . . . . . . . 42

2 Dinâmica do Condensado de Bose-Einstein contendo sólitons $\quad 45$

$2.1 \quad$ Objetivos e motivações . . . . . . . . . . . . . . . . . . . . . . . . 45

$2.2 \quad$ Sistema estudado . . . . . . . . . . . . . . . . . . . . . . . . 46

$3 \quad$ Simulações e Resultados 49

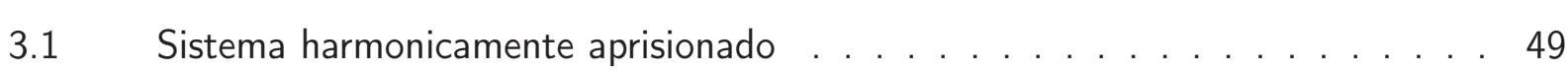

3.1 .1 Condensado puro (modo dipolar) . . . . . . . . . . . . . . . . . . . . . 49

3.1 .2 Condensado + Sóliton . . . . . . . . . . . . . . . . . 52

3.2 Sistema harmonicamente aprisionado com barreira central . . . . . . . . . . 57

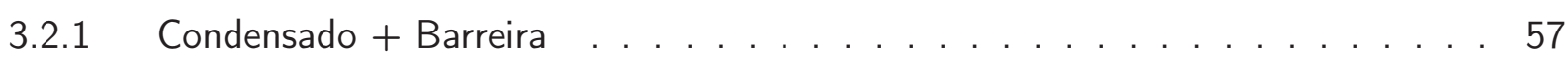


3.2.2 Condensado + Sóliton + Barreira Baixa . . . . . . . . . . . . . . . . . 64

3.2.3 Condensado + Sóliton + Barreira Alta $\ldots \ldots \ldots$. . . . . . . . . 71

3.3 Sistema harmonicamente aprisionado com rugosidade no potencial . . . . . . . 73

3.3 .1 Condensado puro . . . . . . . . . . . . . . . . . . . . . . . . . . . 74

3.3.2 Condensado + Sóliton . . . . . . . . . . . . . . . . . . . 78

4 Comparação com a Teoria de múltiplos orbitais $\quad 85$

$\begin{array}{lll}5 & \text { Conclusão } & 91\end{array}$

$\begin{array}{ll}\text { REFERÊNCIAS } & 93\end{array}$

$\begin{array}{ll}\text { Apêndice A - Constantes Físicas } & 95\end{array}$

Apêndice B - Dedução do modo dipolar $\quad 97$

Apêndice C - Dedução da equação do sóliton 103

Apêndice D - Dedução da energia do sóliton 109

Apêndice E - Cálculo do potencial químico do BEC na presença da barreira através da TFA 


\section{Introdução}

O cientista não é o homem que fornece as verdadeiras respostas, é quem faz as verdadeiras perguntas. Claude Lévi-Strauss

Na natureza existem dois tipos de partículas quânticas, os bósons e os férmions:

- Férmions: Spin semi-inteiro e cada estado quântico só pode ser ocupado por uma única partícula. Exemplos: elétron $\left(e^{-}\right)$, próton $\left(p^{+}\right)$, quarks, neutrinos...

- Bósons: Spin inteiro e cada estado quântico pode ser ocupado por mais de uma partícula. Exemplos: Fóton, Glúon, $\mathrm{He}^{4} \ldots$

O número de ocupação médio dessas partículas num estado $r$ é dada pela seguinte expressão:

$$
\left\langle n_{r}\right\rangle=\frac{1}{\mathrm{e}^{\beta\left(\varepsilon_{r}-\mu\right)} \pm 1}
$$

onde $\beta=\frac{1}{k_{B} T}$ com $k_{B}$ sendo a constante de Boltzmann e $T$ a temperatura, $\varepsilon_{r}$ a energia de uma partícula no estado $r$ e $\mu$ o potencial químico.

Pela definição dada anteriormente, fica evidente que o sinal negativo se refere à densidade de bósons, já que somente nesse tipo de partículas podemos ter o estado $r$ com um número médio de partículas maior que 1 .

\subsection{Condensado de Bose-Einstein}

Dadas essas definiç̃es, vamos entender o que é afinal um condensado de bósons. O condensado de Bose-Einstein (BEC), conforme definição da Física Estatística, é o estado em 
que uma quantidade macroscópica de partículas do gás está no estado fundamental. Esse é um sistema importante pois essa grande quantidade de partículas em um só estado, no ponto de vista da Mecânica Quântica, cria uma uma grande onda de matéria coerente proveniente da sobreposição de todas as ondas de De Broglie de cada uma das partículas do sistema.

Como temos uma quantidade macroscópica de partículas quânticas em um só estado, esses sistemas são excelentes para o estudo dos comportamentos quânticos da matéria. Permitem, por exemplo, o estudo de vórtices quantizados (1) e dos modos coletivos.(2-3) Por isso o interesse em produzi-los experimentalmente foi grande desde sua predição feita em 1925 por Albert Einstein.(4) Finalmente em 1995, a primeira amostra experimental desse sistema foi produzida.(5)

\subsubsection{Gás de bósons não interagentes aprisionados}

Conforme a Física Estatística, temos a seguinte grande função de partição do gás de bósons não interagentes: (6)

$$
\Xi\left(\beta, \varepsilon_{r}, \mu\right)=\prod_{r \in\{\text { estados }\}} \frac{1}{1-\mathrm{e}^{-\beta\left(\varepsilon_{r}-\mu\right)}} \Rightarrow \ln \left[\Xi\left(\beta, \varepsilon_{r}, \xi\right)\right]=-\sum_{r \in\{\text { estados }\}} \ln \left[1-\xi \mathrm{e}^{-\beta \varepsilon_{r}}\right]
$$

sendo $\xi=\mathrm{e}^{\beta \mu}$ a fugacidade do sistema.

Para o número total de partículas, temos:

$$
\begin{gathered}
N=\xi \frac{\partial}{\partial \xi} \ln \left[\Xi\left(\beta, \varepsilon_{r}, \xi\right)\right]=\xi \frac{\partial}{\partial \xi}\left\{-\sum_{r \in\{\text { estados }\}} \ln \left[1-\xi \mathrm{e}^{-\beta \varepsilon_{r}}\right]\right\} \\
N=\sum_{r \in\{\text { estados }\}} \underbrace{\frac{1}{\xi^{-1} \mathrm{e}^{\beta \varepsilon_{r}}-1}}_{\left\langle n_{r}\right\rangle}
\end{gathered}
$$

sendo $\left\langle n_{r}\right\rangle$ o número de ocupação médio do estado $r$.

O potencial químico, num contexto quântico, é negativo para os bósons, mas aumenta com o decréscimo de temperatura até se anular, instante esse que, com uma quantidade macroscópica de partículas no estado fundamental, caracteriza a condensação de Bose-Einstein.(6) 
Quando isso ocorre $(\mu=0)$, temos a fugacidade $\xi=1$ e $\varepsilon_{r} \ll k_{B} T$ (estado fundamental). A figura 1.1 mostra a distribuição de partículas para três valores de $\xi$. Observe que quanto menor o valor da fugacidade (maiores temperaturas), menor a ocupação do estado fundamental, fazendo com que os estados mais excitados passem a ser ocupados e com uma menor quantidade de partículas por estado. Esse é o regime clássico, em que a quantidade de partículas em cada estado é microscópica, fazendo com que o comportamento quântico da matéria não seja mais observável, ou seja, o que chamamos regime clássico é aquele com baixa fugacidade devido à alta temperatura em que a estatística quântica não se manifesta, pois segundo a equação de ocupação descrita acima, o termo -1 do denominar fica irrelevante.

$$
\left.\begin{array}{l}
\mu \rightarrow 0^{-} \\
\frac{\varepsilon_{r}}{k_{B} T} \rightarrow 0
\end{array}\right\} \Rightarrow f^{0}\left(\varepsilon_{0}\right) \equiv\left\langle n_{0}\right\rangle \rightarrow \infty
$$

ou seja, um número infinitamente grande de partículas concentradas no estado fundamental, caracterizando a quantidade macroscópica de partículas citada anteriormente.

Figura 1.1 - Distribuição estatística de partículas para três valores de $\xi$

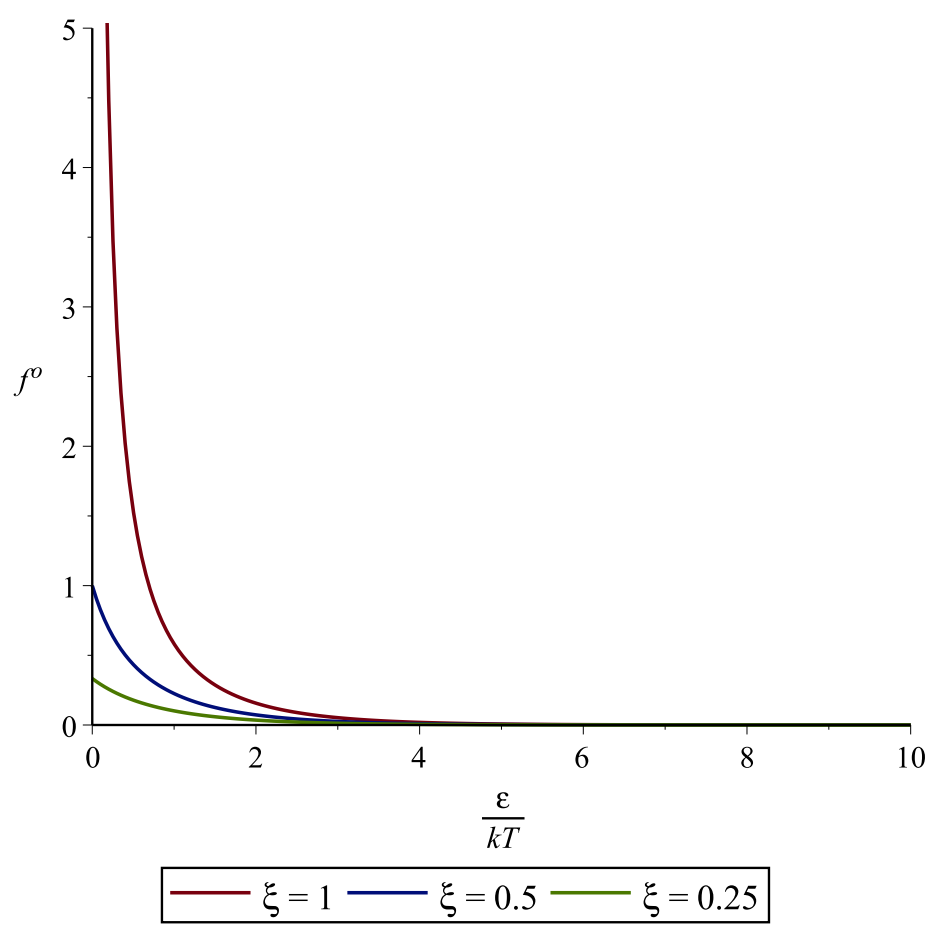

Fonte: Elaborada pelo autor.

Esse sistema é atingido quando a temperatura de um gás de bósons é reduzida abaixo da chamada temperatura crítica ou temperatura de condensação $\left(T_{c}\right)$ : 


$$
T_{c}=C \frac{\hbar^{2} n^{2 / 3}}{m k_{B}}
$$

em que a constante de proporcionalidade $C$ depende do tipo de armadilha usada no aprisionamento, $\hbar=\frac{h}{2 \pi}$ é a constante de Planck reduzida, $n$ é a densidade numérica de partículas, $m$ é a massa de cada partícula e $k_{B}$ é a constante de Boltzmann. Para esse cálculo, utilizamos a degenerescência dos estados quânticos. Para a armadilha harmônica, comumente utilizada nos experimentos, caracterizada pelo seguinte potencial de aprisionamento:

$$
V(x, y, z)=\frac{1}{2} m \omega_{x}^{2} x^{2}+\frac{1}{2} m \omega_{y}^{2} y^{2}+\frac{1}{2} m \omega_{z}^{2} z^{2}
$$

Temos a energia dos estados quânticos caracterizados pelos números quânticos $n_{x}, n_{y}, n_{z}$, dada por:

$$
\varepsilon\left(n_{x}, n_{y}, n_{z}\right)=\left(n_{x}+\frac{1}{2}\right) \hbar \omega_{x}+\left(n_{y}+\frac{1}{2}\right) \hbar \omega_{y}+\left(n_{z}+\frac{1}{2}\right) \hbar \omega_{z}
$$

onde $\hbar$ é a constante de Planck reduzida.

Disso, podemos obter o número de partículas com energia menor ou igual a $\varepsilon=\varepsilon_{x}+\varepsilon_{y}+\varepsilon_{z}$, considerando $\varepsilon \gg \hbar \omega_{i}$ e portanto $\varepsilon$ contínuo:

$$
\begin{gathered}
G(\varepsilon)=\frac{1}{\left(\hbar \omega_{x}\right)\left(\hbar \omega_{y}\right)\left(\hbar \omega_{z}\right)} \int_{0}^{\varepsilon} d \varepsilon_{x} \int_{0}^{\varepsilon-\varepsilon_{x}} d \varepsilon_{y} \int_{0}^{\varepsilon-\varepsilon_{x}-\varepsilon_{y}} d \varepsilon_{z} \\
G(\varepsilon)=\frac{\varepsilon^{3}}{6 \hbar^{3} \omega_{x} \omega_{y} \omega_{z}}
\end{gathered}
$$

Para a degenerescência, isto é, o número de partículas com energia entre $\varepsilon$ e $\varepsilon+d \varepsilon$, temos:

$$
g(\varepsilon)=\frac{d}{d \varepsilon} G(\varepsilon)=\frac{\varepsilon^{2}}{2 \hbar^{3} \omega_{x} \omega_{y} \omega_{z}}=C_{3} \varepsilon^{2}
$$

$\operatorname{com} C_{3}=\frac{1}{2 \hbar^{3} \omega_{x} \omega_{y} \omega_{z}}$

Para uma definição mais precisa de temperatura crítica, temos que ela ocorre no limite em que os estados excitados ficam saturados, sobrando apenas o estado fundamental para que os átomos ocupem. Nessa situação, o potencial químico assume seu valor máximo $(\mu=0)$ : 


$$
N=N_{e x}\left(T_{c}, \mu=0\right)=\int_{0}^{\infty} g(\varepsilon)\langle n\rangle d \varepsilon=\int_{0}^{\infty} \frac{C_{3} \varepsilon^{2}}{\mathrm{e}^{\frac{\varepsilon}{k_{B} T_{c}}}-1} d \varepsilon
$$

sendo $N_{e x}$ o número de partículas nos estados excitados,

Fazendo $\epsilon=\frac{\varepsilon}{k_{B} T_{c}}$ :

$$
N=C_{3}\left(k_{B} T_{c}\right)^{3} \int_{0}^{\infty} \frac{\epsilon^{2}}{\mathrm{e}^{\epsilon}-1} d \epsilon=C_{3}\left(k_{B} T_{c}\right)^{3} \Gamma(3) \zeta(3)
$$

sendo $\Gamma(x)$ a função gama e $\zeta(x)$ a função zeta de Riemann.

Substituindo $T_{c}=C \frac{\hbar^{2} n^{2 / 3}}{m k_{B}}$, temos:

$$
C=\left[\frac{2}{\Gamma(3) \zeta(3)}\right]^{1 / 3} \approx 0,94 \Rightarrow T_{c} \approx 9,87 \times 10^{-25} \bar{\omega} N^{1 / 3}(n K)
$$

\subsubsection{Fração condensada}

Definimos como fração condensada a fração dos átomos presentes no gás, que faz parte do BEC, ou seja, que estão no estado fundamental:

$$
\frac{N_{0}}{N}=\frac{N-N_{e x}}{N}=1-\frac{N_{e x}}{N}=1-\frac{C_{3}\left(k_{B} T\right)^{3} \Gamma(3) \zeta(3)}{C_{3}\left(k_{B} T_{c}\right)^{3} \Gamma(3) \zeta(3)}
$$

Portanto:

$$
N_{0}(T)=N\left[1-\left(\frac{T}{T_{c}}\right)^{3}\right]
$$

\subsubsection{Equação de Gross-Pitaevskii (GPE)}

Na seção anterior descrevemos os gases de bósons não interagentes, isto é, gases ideais. Mas queremos estudar gases reais, ou seja, gases em que haja interação entre as partículas. Para isso usamos a teoria de campo-médio de Gross-Pitaevskii. Essa teoria descreve as pro- 
priedades de um gás de bósons interagente no zero absoluto de temperatura com o fator de espalhamento muito menor que a distância média entre as partículas do gás, o que garante que a perda de átomos do BEC para os demais estados quânticos do sistema seja desprezível.

Considerando um sistema de $N$ partículas bosônicas descrito pelo hamiltoniano:

$$
H=\sum_{i=0}^{N}\left[\frac{p_{i}^{2}}{2 m_{i}}+V\left(\vec{r}_{i}\right)+U_{0} \sum_{i<j} \delta\left(\vec{r}_{i}-\vec{r}_{j}\right)\right]
$$

onde temos a energia cinética (primeiro termo), potencial externo (segundo termo) e potencial de interação entre partículas (terceiro termo), sendo $U_{0}$ a constante que caracteriza o potencial de contato efetivo entre as partículas. Consideraremos aqui interações repulsivas adotando $U_{0}>0$.

Em gases atômicos diluídos as interações são primariamente binárias e podem ser tratadas teoricamente como uma aproximação de espalhamento de onda s. A forma exata do potencial de interação entre partículas é ignorada e apenas um parâmetro, $a$, o comprimento de espalhamento da onda s, caracteriza a interação, onde $U_{0}=\frac{4 \pi \hbar^{2} a}{m}$. Vamos criar o operador hamiltoniano:

$$
\hat{H}=\sum_{i=0}^{N}\left[-\frac{\hbar^{2}}{2 m_{i}} \nabla_{i}^{2}+V\left(\vec{r}_{i}\right)+U_{0} \sum_{i<j} \delta\left(\vec{r}_{i}-\vec{r}_{j}\right)\right]
$$

Em seguida obtemos o funcional da energia para esse sistema de $N$ partículas:

$$
E=\left\langle\Phi\left(\vec{r}_{1} \cdots \vec{r}_{n}\right)|\hat{H}| \Phi\left(\vec{r}_{1} \cdots \vec{r}_{n}\right)\right\rangle=\int d \vec{r}_{1} \cdots \int d \vec{r}_{n} \Phi^{*}\left(\vec{r}_{1} \cdots \vec{r}_{n}\right) \hat{H} \Phi\left(\vec{r}_{1} \cdots \vec{r}_{n}\right)
$$

$$
\begin{aligned}
E & =\int d \vec{r}_{1} \cdots \int d \vec{r}_{n} \Phi^{*}\left(\vec{r}_{1} \cdots \vec{r}_{n}\right)\left\{\sum_{i=0}^{N}\left[-\frac{\hbar^{2}}{2 m_{i}} \nabla_{i}^{2}+V\left(\vec{r}_{i}\right)+U_{0} \sum_{i<j} \delta\left(\vec{r}_{i}-\vec{r}_{j}\right)\right]\right\} \Phi\left(\vec{r}_{1} \cdots \vec{r}_{n}\right) \\
& =\sum_{i=0}^{N} \int d \vec{r}_{1} \cdots \int d \vec{r}_{n} \Phi^{*}\left(\vec{r}_{1} \cdots \vec{r}_{n}\right)\left[-\frac{\hbar^{2}}{2 m_{i}} \nabla_{i}^{2}+V\left(\vec{r}_{i}\right)+U_{0} \sum_{i<j} \delta\left(\vec{r}_{i}-\vec{r}_{j}\right)\right] \Phi\left(\vec{r}_{1} \cdots \vec{r}_{n}\right)
\end{aligned}
$$

Utilizando a aproximação de Hartree, isto é: 


$$
\Phi\left(\vec{r}_{1} \cdots \vec{r}_{n}\right)=\varphi\left(\vec{r}_{1}\right) \cdots \varphi\left(\vec{r}_{n}\right)
$$

sendo $\varphi\left(\vec{r}_{i}\right)$ a função de onda da i-ésima partícula, solução do hamiltoniano sem o termo de interação, temos:

$$
E=\sum_{i=0}^{N} \int d \vec{r}_{1} \cdots \int d \vec{r}_{n}\left[\varphi\left(\vec{r}_{1}\right) \cdots \varphi\left(\vec{r}_{n}\right)\right]^{*}\left[-\frac{\hbar^{2}}{2 m_{i}} \nabla_{i}^{2}+V\left(\vec{r}_{i}\right)+U_{0} \sum_{i<j} \delta\left(\vec{r}_{i}-\vec{r}_{j}\right)\right]\left[\varphi\left(\vec{r}_{1}\right) \cdots \varphi\left(\vec{r}_{n}\right)\right]
$$

$$
\begin{aligned}
E & =\sum_{i=0}^{N} \int d \vec{r}_{i} \varphi^{*}\left(\vec{r}_{i}\right)\left[\frac{-\hbar^{2}}{2 m_{i}} \nabla_{i}^{2}+V\left(\vec{r}_{i}\right)+U_{0} \sum_{i<j} \int \delta\left(\vec{r}_{i}-\vec{r}_{j}\right)\left|\varphi\left(\vec{r}_{j}\right)\right|^{2} d \vec{r}_{j}\right] \varphi\left(\vec{r}_{i}\right) \\
& =\sum_{i=0}^{N} \int d \vec{r}_{i}\left[-\frac{\hbar^{2}}{2 m_{i}} \varphi^{*}\left(\vec{r}_{i}\right) \nabla_{i}^{2} \varphi\left(\vec{r}_{i}\right)+V\left(\vec{r}_{i}\right) \varphi^{*}\left(\vec{r}_{i}\right) \varphi\left(\vec{r}_{i}\right)+\frac{N-1}{2} U_{0}\left|\varphi\left(\vec{r}_{i}\right)\right|^{2} \varphi^{*}\left(\vec{r}_{i}\right) \varphi\left(\vec{r}_{i}\right)\right]
\end{aligned}
$$

Mas:

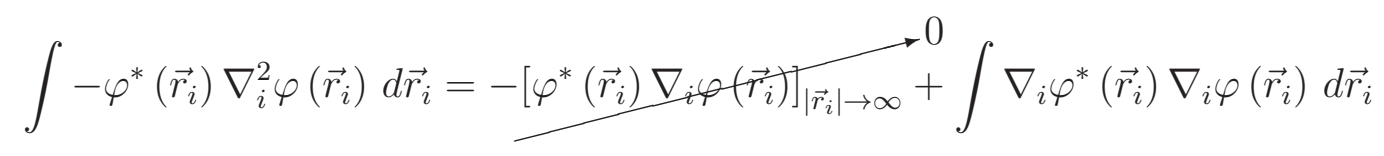

Então:

$$
\begin{aligned}
E & =\sum_{i=0}^{N} \int d \vec{r}_{i}\left[\frac{\hbar^{2}}{2 m_{i}} \nabla_{i} \varphi^{*}\left(\vec{r}_{i}\right) \nabla_{i} \varphi\left(\vec{r}_{i}\right)+V\left(\vec{r}_{i}\right) \varphi^{*}\left(\vec{r}_{i}\right) \varphi\left(\vec{r}_{i}\right)+\frac{N-1}{2} U_{0}\left|\varphi\left(\vec{r}_{i}\right)\right|^{2} \varphi^{*}\left(\vec{r}_{i}\right) \varphi\left(\vec{r}_{i}\right)\right] \\
& =N \int d \vec{r}\left[\frac{\hbar^{2}}{2 m}|\nabla \varphi(\vec{r})|^{2}+V(\vec{r})|\varphi(\vec{r})|^{2}+\frac{N-1}{2} U_{0}|\varphi(\vec{r})|^{2}|\varphi(\vec{r})|^{2}\right]
\end{aligned}
$$

Finalmente:

$$
E=N \int\left[\frac{\hbar^{2}}{2 m}|\nabla \varphi(\vec{r})|^{2}+V(\vec{r})|\varphi(\vec{r})|^{2}+\frac{N-1}{2} U_{0}|\varphi(\vec{r})|^{4}\right] d \vec{r}
$$

Se definirmos a função de onda do condensado como um todo: 


$$
\psi(\vec{r})=\sqrt{N} \varphi(\vec{r}) \Rightarrow n(\vec{r})=|\psi(\vec{r})|^{2}
$$

sendo $n(\vec{r})$ a densidade numérica espacial e utilizarmos $N$ grande $(N-1 \approx N)$, temos:

$$
\begin{aligned}
N & =\int|\psi(\vec{r})|^{2} d \vec{r} \\
E & =\int\left[\frac{\hbar^{2}}{2 m}|\nabla \psi(\vec{r})|^{2}+V(\vec{r})|\psi(\vec{r})|^{2}+\frac{1}{2} U_{0}|\psi(\vec{r})|^{4}\right] d \vec{r} \\
& =\int\left[-\frac{\hbar^{2}}{2 m} \psi^{*} \nabla^{2} \psi(\vec{r})+V(\vec{r}) \psi^{*}(\vec{r}) \psi(\vec{r})+\frac{1}{2} U_{0}\left[\psi^{*}(\vec{r}) \psi(\vec{r})\right]^{2}\right] d \vec{r}
\end{aligned}
$$

Tomando $L=E-\mu N$, temos:

$L=\int\left\{-\frac{\hbar^{2}}{2 m} \psi^{*} \nabla^{2} \psi(\vec{r})+[V(\vec{r})-\mu] \psi^{*}(\vec{r}) \psi(\vec{r})+\frac{1}{2} U_{0}\left[\psi^{*}(\vec{r}) \psi(\vec{r})\right]^{2}\right\} d \vec{r}$

Para finalizar, utilizamos o método de Lagrange para minimizar a energia com respeito à variação de $\psi^{*}$ mantendo o número de partículas constante, onde $\mu$ é o potencial químico (energia necessária para adicionar uma nova partícula ao sistema):

$$
\begin{gathered}
\frac{\partial L}{\partial \psi^{*}}=\frac{d}{d t}\left(\frac{\partial L}{\partial \dot{\psi}^{*}}\right)=0 \\
-\frac{\hbar^{2}}{2 m} \nabla^{2} \psi(\vec{r})+[V(\vec{r})-\mu] \psi(\vec{r})+U_{0}|\psi(\vec{r})|^{2} \psi(\vec{r})=0 \\
-\frac{\hbar^{2}}{2 m} \nabla^{2} \psi(\vec{r})+V(\vec{r}) \psi(\vec{r})+U_{0}|\psi(\vec{r})|^{2} \psi(\vec{r})=\mu \psi(\vec{r})
\end{gathered}
$$

Essa é a Equação de Gross-Pitaevskii independente do tempo. Para a dedução dessa equação, assumimos que o BEC pode ser descrito por apenas uma função de onda, por isso essa é uma aproximação de campo médio.

Essa teoria é uma aproximação e portanto não é válida sempre. A partir do momento em que a perda de átomos para os demais estados quânticos do sistema é muito significativa essa não é mais uma aproximação válida. Perceba que ao longo da dedução, assumimos que a 
única interação existente entre as partículas é a interação de contato, isto é, essa aproximação é valida para gases diluídos $\left(n a^{3} \ll 1\right)$ no zero absoluto de temperatura $(T=0 K)$.

\subsubsection{Aproximação de Thomas-Fermi (TFA)}

O sistema de interesse para qualquer pesquisa (mesmo as teóricas) é aquele que podemos obter experimentalmente. Para criarmos um BEC, precisamos resfirar um gás de bósons, retirando sua energia de vibração, ou seja, sua energia cinética. Desta forma, ao final do processo, teremos um condensado em que a energia de interação entre partículas e o potencial externo são muito maiores que a energia cinética, de tal forma que podemos desconsiderar o termo cinético. A aproximação de Thomas-Fermi é aquela em que desprezamos o termo cinético devido à forte interação entre partículas ou ao elevado número de partículas,(7) o que, para o aprisionamento harmônico, corresponde a altos valores da seguinte razão:

$$
u=\frac{8 \pi a N}{l_{\text {osc }}}
$$

onde $l_{\text {osc }}=\sqrt{\frac{\hbar}{m \omega_{o s c}}}$ é o comprimento do oscilador. Nesse regime, devido à forte interação temos perfis de densidade muito largos e aproximadamente parabólicos, de forma que na maior parte da função de onda, temos um gradiente de densidade muito pequeno, o que faz com que o termo cinético $\left(\nabla^{2} \psi\right)$ possa ser desprezado frente ao termo de interação.

$$
\begin{gathered}
=\frac{\hbar^{2}}{2 m} \nabla^{2} \psi(\vec{r})+V(\vec{r}) \psi(\vec{r})+U_{0}|\psi(\vec{r})|^{2} \psi(\vec{r})=\mu \psi(\vec{r}) \\
n(\vec{r})=|\psi(\vec{r})|^{2}=\frac{\mu-V(\vec{r})}{U_{0}}
\end{gathered}
$$

Vamos definir como raio de Thomas-Fermi o raio da nuvem atômica quando usamos a aproximação de Thomas-Fermi em relação ao pico da densidade:

$$
R_{T F}=\left|V^{-1}(\mu)\right|
$$

Para obtermos o valor de $\mu$, vamos utilizar o fato de que a GPE implica conservação do número de partículas e esse número pode ser calculado pela integral da densidade numérica ao longo de todo o condensado. Dessa forma obteremos o valor de $\mu=$ cte para nosso sistema 
resolvendo a seguinte equação na variável $\mu$ :

$$
N=\int_{|\vec{r}|<R_{T F}} n(\vec{r}) d \vec{r}=\frac{1}{U_{0}} \int_{|\vec{r}|<R_{T F}} \mu-V(\vec{r}) d \vec{r}
$$

\subsubsection{Equação dependente do tempo}

Assim como a GPE independente do tempo, a dependente do tempo pode ser deduzida por método variacional, utilizando a Lagrangiana adequada para sistemas dependentes do tempo, contendo um termo de fluxo da função de onda, e o princípio da mínima ação:

$$
\begin{gathered}
L=\int \frac{i}{2} \hbar\left(\psi^{*} \frac{\partial \psi}{\partial t}-\psi \frac{\partial \psi^{*}}{\partial t}\right)-\left[\frac{\hbar^{2}}{2 m}|\nabla \psi|^{2}+V(\vec{r}, t)|\psi|^{2}+\frac{1}{2} U_{0}|\psi|^{4}\right] d \vec{r} \\
=-\int \frac{i}{2} \hbar\left(\psi \dot{\psi}^{*}-\psi^{*} \dot{\psi}\right)-\frac{\hbar^{2}}{2 m} \psi^{*} \nabla^{2} \psi+V(\vec{r}, t) \psi^{*} \psi+\frac{1}{2} U_{0}\left(\psi^{*} \psi\right)^{2} d \vec{r} \\
\frac{\partial L}{\partial \psi^{*}}=\frac{d}{d t}\left(\frac{\partial L}{\partial \dot{\psi}^{*}}\right) \\
\int-\frac{i}{2} \hbar \dot{\psi}-\frac{\hbar^{2}}{2 m} \nabla^{2} \psi(\vec{r})+V(\vec{r}) \psi(\vec{r})+U_{0}|\psi(\vec{r})|^{2} \psi(\vec{r})-\frac{i}{2} \hbar \dot{\psi} d \vec{r}=0 \\
-\frac{\hbar^{2}}{2 m} \nabla^{2} \psi(\vec{r}, t)+V(\vec{r}, t) \psi(\vec{r}, t)+U_{0}|\psi(\vec{r}, t)|^{2} \psi(\vec{r}, t)=i \hbar \frac{\partial}{\partial t} \psi(\vec{r}, t)
\end{gathered}
$$

\subsubsection{Modos coletivos}

Em sistemas de muitos corpos, o estudo dos modos normais e dos modos coletivos (modos normais de baixa energia do conjunto de partículas) são de essencial importância, já que o movimento geral do sistema é descrito como uma combinação dos modos normais do mesmo, dessa forma, o estudo dos modos é uma forma de dividir um movimento complexo em partes 
mais simples. Essas oscilações são descritas pelas soluções linearizadas da GPE dependente do tempo.

$$
-\frac{\hbar^{2}}{2 m} \nabla^{2} \psi(\vec{r}, t)+V(\vec{r}, t) \psi(\vec{r}, t)+U_{0}|\psi(\vec{r}, t)|^{2} \psi(\vec{r}, t)=i \hbar \frac{\partial}{\partial t} \psi(\vec{r}, t)
$$

Entre vários modos coletivos existentes, daremos atenção especial ao modo de mais baixa energia, o dipolar, que corresponde ao movimento do centro de massa do sistema devido à armadilha harmônica e tem frequências iguais às da armadilha em cada uma das direções espaciais. A dedução desse fato para o caso unidimensional está no apêndice $B$, que resulta na seguinte equação para o movimento do centro de massa do BEC:

$$
x_{0}(t)=x_{C M} \cos (\omega t)
$$

Dentre os outros modos está o breathing mode, que é o modo em que o raio do condensado varia no tempo.

Uma forma de se obter as frequências desses modos é através de cálculo variacional, utilizando a lagrangiana obtida através de uma função teste adequada. Um exemplo do uso desse método está descrito no apêndice $B$ citado acima para o cálculo da frequência do modo dipolar. Perceba que a partir dessa mesma função de onda teste, pode-se calcular a frequência do breathing mode impondo que apenas $\alpha, \beta$ e $w$ variem, obtendo a frequência $\omega \sqrt{3}$ para sistemas de campo médio.(3)

\subsubsection{Sistema unidimensional}

Nesse trabalho exploraremos somente sistemas unidimensionais no regime de ThomasFermi e usando potenciais harmônicos. Vamos começar por definir o potencial de aprisionamento dos átomos:

$$
V(x)=\frac{1}{2} m \omega^{2} x^{2}
$$

Em seguida, a partir das GPEs dimensional e adimensional, vamos determinar os coeficientes adimensionais das equações (dependente e independente do tempo) utilizadas nas 
simulações numéricas:

$$
\begin{gathered}
-\frac{\hbar^{2}}{2 m} \frac{d^{2}}{d x^{2}} \psi(x)+\frac{1}{2} m \omega^{2} x^{2} \psi(x)+U_{0}|\psi(x)|^{2} \psi(x)=\mu \psi(x) \\
\overbrace{-\frac{\hbar^{2}}{2 m} \frac{\partial^{2}}{\partial x^{2}} \psi(x, t)+\frac{1}{2} m \omega^{2} x^{2} \psi(x, t)+U_{0}|\psi(x, t)|^{2} \psi(x, t)=i \hbar \frac{\partial}{\partial t} \psi(x, t)}^{-\frac{1}{2} \frac{d^{2}}{d x^{2}} \psi(x)+\frac{1}{2} \omega^{2} x^{2} \psi(x)+G_{0}|\psi(x)|^{2} \psi(x)=\mu_{0} \psi(x)} \\
-\frac{1}{2} \frac{\partial^{2}}{\partial x^{2}} \psi(x, t)+\frac{1}{2} \omega^{2} x^{2} \psi(x, t)+G_{0}|\psi(x, t)|^{2} \psi(x, t)=i \frac{\partial}{\partial t} \psi(x, t)
\end{gathered}
$$

Ou seja:

$$
x \rightarrow \frac{x}{L_{0}} t \rightarrow\left\{\begin{array}{l}
1=\frac{\hbar}{m \omega_{0} L_{0}^{2}} \\
\frac{m L_{0}^{2}}{\hbar \omega_{0}}=1 \\
G_{0}=\frac{U_{0}}{\hbar \omega_{0}} \\
\mu_{0}=\frac{\mu}{\hbar \omega_{0}}
\end{array}\right.
$$

Então, temos:

$$
\begin{aligned}
& \omega_{0}=1 \\
& L_{0}=\sqrt{\frac{\hbar}{m \omega_{0}}} \\
& G_{0}=\frac{U_{0}}{\hbar \omega_{0}} \\
& \mu_{0}=\frac{\mu}{\hbar \omega_{0}}
\end{aligned}
$$

Para termos uma notação limpa, vamos manter os nomes das variáveis anteriores $(x, t$ e $\mu)$, mas lembrando que a partir de agora usaremos apenas equações com variáveis adimensionais. 
Em posse das equações dependente e independente do tempo, vamos seguir para a TFA para calcularmos a densidade aproximada, o raio de Thomas-Fermi e o equivalente potencial químico. Vamos começar pela densidade:

$$
n(x)=|\psi(x)|^{2}=\frac{\mu-\frac{1}{2} \omega^{2} x^{2}}{G_{0}}
$$

Seguindo para o raio de Thomas-Fermi:

$$
R_{T F}=\left|V^{-1}(\mu)\right|=\frac{\sqrt{2 \mu}}{\omega}
$$

Agora vamos obter o potencial químico para que possamos determinar esses resultados em função somente dos parâmetros $\omega$ e $G_{0}$ :

$$
\begin{aligned}
N=\int_{|\vec{r}|<R_{T F}} n(\vec{r}) d \vec{r} & =\int_{-R}^{R} \frac{\mu-\frac{1}{2} \omega^{2} x^{2}}{G_{0}} d x \\
& =\frac{1}{G_{0}}\left[\mu x-\frac{1}{6} \omega^{2} x^{3}\right]_{-R}^{R} \\
& =\frac{1}{G_{0}}\left(2 \mu R-\frac{1}{3} \omega^{2} R^{3}\right)
\end{aligned}
$$

Mas $R=\frac{\sqrt{2 \mu}}{\omega}$ :

$$
N=\frac{(2 \mu)^{3 / 2}}{\omega G_{0}}\left(1-\frac{1}{3}\right)
$$

Portanto:

$$
\mu=\frac{1}{2}\left(\frac{3}{2} N \omega G_{0}\right)^{2 / 3}
$$

Mas a função de onda é normalizada pelo número de partículas:

$$
\mu=\frac{1}{2}\left(\frac{3}{2} \omega G_{0}\right)^{2 / 3}
$$

Substituindo nas expressões da densidade e do raio de Thomas-Fermi: 


$$
\begin{gathered}
R=\frac{\sqrt{2 \mu}}{\omega} \\
R=\left(\frac{3}{2} \frac{G_{0}}{\omega^{2}}\right)^{1 / 3}
\end{gathered}
$$

\subsection{Estados Excitados}

Outros aspectos muito estudados dos condensados são os estados excitados desse sistema, que incluem sólitons (estáveis somente em sistemas unidimensionais e quasi-unidimensionais) e vórtices. (4, 8) Nesse trabalho vamos estudar apenas sistemas unidimensionais, isto é, estudaremos apenas sólitons e sua influência sobre a dinâmica dos BECs.

\subsubsection{Sólitons}

Um sóliton é uma estrutura unidimensional que é solução da GPE dependente do tempo sem o termo de potencial, (8) isto é:

$$
-\frac{\hbar^{2}}{2 m} \nabla^{2} \psi(\vec{r}, t)+U_{0}|\psi(\vec{r}, t)|^{2} \psi(\vec{r}, t)=i \hbar \frac{\partial}{\partial t} \psi(\vec{r}, t)
$$

No formato adimensional unidimensional:

$$
-\frac{1}{2} \frac{\partial^{2}}{\partial x^{2}} \psi(x, t)+G_{0}|\psi(x, t)|^{2} \psi(x, t)=i \frac{\partial}{\partial t} \psi(x, t)
$$

Nesse caso, para que tenhamos uma estrutura estável precisamos que a dispersão e não linearidade do sistema se equilibrem, isto é, essa estrutura é resultado do equilíbrio entre a interação das partículas do BEC e a energia de movimento das mesmas e se mostra dentro de um BEC como um pacote de onda não linear que se movimenta e não se deforma nem muda sua velocidade com o tempo. Essa estrutura é chamada onda solitária, ou Sóliton.

O sóliton é definido como um pacote de onda de forma permanente e localização muito bem definida. Em um BEC, podem assumir duas formas dependendo do tipo de interação entre as partículas: dark solitons (depressão de densidade, ocorre em condensados repul- 
sivos) e bright solitons (ascensão de densidade, ocorre em condensados atrativos). Nesse trabalho estudaremos somente os condensados repulsivos, ou seja, estudaremos somente os dark solitons.

Sabemos que o dark soliton é uma onda cuja forma não se altera no tempo e que obedece à equação descrita acima, então podemos calcular uma função que o descreve (apêndice C):

$$
\psi_{0}=\sqrt{n} \varphi_{\text {soliton }}=\sqrt{n}\left[\sqrt{1-u^{2}} \operatorname{tgh}\left(\frac{x-x_{0}}{\xi} \sqrt{1-u^{2}}\right)+i u\right]
$$

onde $u=\frac{v}{c}$, com $v(0 \leq v \leq c)$ é a velocidade do sóliton e $c=\sqrt{n G_{0}}$ é a velocidade do som no meio estudado, $\xi$ é o healing lenght (comprimento característico do sóliton) e $x_{0}$ é a posição do sóliton.

Vamos analisar essa equação para ver algumas propriedades dos sólitons relativas à densidade e à fase da função de onda. Primeiramente vamos analisar a densidade:

$$
\left|\varphi_{\text {soliton }}(x, u)\right|^{2}=\left(1-u^{2}\right) \operatorname{tgh}^{2}\left(\frac{x-x_{0}}{\xi} \sqrt{1-u^{2}}\right)+u^{2}
$$

Observe que quanto menor a velocidade do sóliton, menor a densidade do seu core ( $\left.n\left(x_{0}, u\right)=u^{2}\right)$, sendo que para seu maior valor $(u=1)$, não há depressão de densidade e para seu menor valor ( $u=0$, black soliton), a depressão é máxima, atingindo densidade nula no core. Em relação à fase da função de onda, sabemos que:

$$
\theta(x, u)=\operatorname{arctg}\left(\frac{\Im\left\{\varphi_{\text {soliton }}\right\}}{\Re\left\{\varphi_{\text {soliton }}\right\}}\right)=\operatorname{arctg}\left[\frac{u}{\sqrt{1-u^{2}} \operatorname{tgh}\left(\frac{x-x_{0}}{\xi} \sqrt{1-u^{2}}\right)}\right]
$$

Observe que há uma diferença abrupta de fase no core do sóliton, isto é, $\theta\left(x_{0}^{ \pm}, u>0\right)=$ $\pm \frac{\pi}{2}$. Essas são as propriedades diretas da equação do sóliton: depressão de densidade com abrupta diferença de fase $(\Delta \theta=\pi)$.

O próximo passo é calcular a energia do sóliton, que pode ser obtida através do funcional de energia, que é simplesmente a diferença entre o funcional com o sóliton e sem ele (apêndice D):

$$
E_{\text {soliton }}=\frac{4}{3} n c\left(1-u^{2}\right)^{3 / 2}
$$


Uma coisa importante que podemos observar nesse resultado é o comportamento antidamping do sóliton, isto é, quanto menor sua energia, maior sua velocidade, ou seja, perdas de energia geram ganho de velocidade, justamente o contrário do que ocorre com uma partícula. Por isso e pelo fato do sóliton se manter coerente é comum uma analogia do sóliton com uma partícula de massa negativa $(m \sim-4 n c)$, em que sua energia diminuiria com o aumento da velocidade.

Se considerarmos conservação de energia para esse sistema, obteremos, conforme a expressão de energia obtida, velocidade constante, que é a hipótese que assumimos inicialmente:

$$
\begin{gathered}
\frac{d E_{\text {soliton }}}{d t}=0=\frac{4}{3} n c \frac{d}{d t}\left[\left(1-\frac{\dot{x}_{0}^{2}}{c^{2}}\right)^{3 / 2}\right] \\
\frac{3}{2}\left(1-\frac{\dot{x}_{0}^{2}}{c^{2}}\right)^{1 / 2}\left(-2 \frac{\dot{x}_{0} \ddot{x}_{0}}{c^{2}}\right)=0 \\
\ddot{x}_{0}=0 \Rightarrow x_{0}(t)=v t
\end{gathered}
$$

\subsubsection{Sólitons em BECs aprisionados}

Os sólitons, como visto acima, são soluções da GPE 1D sem potencial externo e, portanto, estáveis nesse sistema, mas esse não é um sistema de interesse, pois não o podemos obter experimentalmente, afinal temos que ter o BEC aprisionado em três dimensões para podermos estudá-lo, e isso requer um potencial externo além de duas dimensões extras. Isso faz com que tenhamos agora uma entidade instável, mas seu tempo de vida pode ser bem grande em sistemas quasi-unidimensionais, obtidos através de um potencial harmônico tridimensional, sendo duas frequências de aprisionamento muito maiores que a terceira, de forma que esse sistema tridimensional se assemelhe muito com um sistema unidimensional (onde os sólitons são estáveis), fazendo com que o tempo de vida se torne muito grande a ponto de podemos estudá-los.

Os sólitons são estáveis apenas em sistemas unidimensionais no zero absoluto de temperatura pois para que isso aconteça temos que impedir que ocorram excitações dos modos transversais do BEC. Para que tenhamos uma boa aproximação em um sistema quasiunidimensional, o tamanho do BEC na direção radial deve ser menor que o healing lenght e a 
temperatura deve ser bem baixa.

Vamos agora calcular a energia para o sóliton em movimento em um BEC aprisionado em uma armadilha harmônica. Para isso, já que a escala da dimensão do sóliton é muito menor que da dimensão do BEC, vamos considerar um potencial químico local dada a inomogeneidade da densidade do BEC aprisionado:(9)

$$
\mu \rightarrow \mu-V\left(x_{0}\right)
$$

Essas considerações nos levam à seguinte energia para um sóliton em um BEC aprisionado:

$$
E_{\text {soliton }}=\frac{4}{3} n c\left[1-u^{2}-\frac{V\left(x_{0}\right)}{c^{2}}\right]^{3 / 2}
$$

Se tomarmos $V(x)=0$, isto é, ausência de potencial externo, retornaremos ao resultado anterior.

A partir desse resultado, da mesma forma que fizemos para o sóliton sem potencial, vamos calcular o movimento do sóliton com a presença de um potencial externo:

$$
\begin{gathered}
\frac{d E_{\text {soliton }}}{d t}=0=\frac{4}{3} n c \frac{d}{d t}\left[\left(1-\frac{\dot{x}_{0}^{2}}{c^{2}}-\frac{V\left(x_{0}\right)}{c^{2}}\right)^{3 / 2}\right] \\
\frac{3}{2}\left(1-\frac{\dot{x}_{0}^{2}}{c^{2}}-\frac{V\left(x_{0}\right)}{c^{2}}\right)^{1 / 2}\left(-2 \frac{\dot{x}_{0} \ddot{x}_{0}}{c^{2}}-\frac{\dot{x}_{0}}{c^{2}} \frac{d V}{d x_{0}}\right)=0 \\
\ddot{x}_{0}+\frac{1}{2} \frac{d V}{d x_{0}}=0
\end{gathered}
$$

Vamos tratar a situação que é de nosso interesse, isto é, potencial harmônico:

$$
V(x)=\frac{1}{2} \omega^{2} x^{2} \Rightarrow \ddot{x}_{0}+\left(\frac{\omega}{\sqrt{2}}\right)^{2} x=0
$$

isto é, temos para o sóliton um movimento oscilante com frequência $\omega_{\text {sol }}=\frac{\omega}{\sqrt{2}}$, sendo $\omega$ a frequência do potencial harmônico externo. 


\subsection{Teoria de Múltiplos Orbitais}

O que vimos até agora, ou seja, a teoria de campo médio de Gross-Pitaevskii, que assume que todas as partículas estão em apenas um estado, é amplamente utilizada, porém existem outras teorias que tentam explicar melhor o BEC, considerando-o como um sistema de muitos corpos e múltiplos orbitais. Uma delas é a MCTDHB(M) (Multi Configurational Time-Dependent Hartree method for Bosons with M orbitals), (10) teoria de múltiplos orbitais que basicamente estuda o BEC como um sistema de muitos corpos levando em considerações múltiplos estados excitados ao invés de utilizar uma função de onda média, como fazemos na teoria de campo médio de Gross-Pitaevskii.

Dessa forma, assim como na teoria de campo médio, temos o seguinte hamiltoniano para o sistema:

$$
H=\sum_{i=0}^{N}\left[\frac{p_{i}^{2}}{2 m_{i}}+V\left(\vec{r}_{i}\right)+U_{0} \sum_{i<j} \delta\left(\vec{r}_{i}-\vec{r}_{j}\right)\right]
$$

Mas para esse caso vamos trabalhar com um sistema de $N$ bósons. Vamos começar definindo as funções de onda utilizadas em nosso sistema. Usaremos funções de onda para descrevermos um conjunto completo de orbitais $\left\{\varphi_{k}(\vec{r}, t)\right\}$ ortonormalizados:

$$
\int \varphi_{k}^{*}(\vec{r}, t) \varphi_{j}(\vec{r}, t) d \vec{r}=\delta_{k j}
$$

Para os próximos passos, vamos trabalhar com segunda quantização, utilizando os operadores de criação e destruição, que para os bósons obedecem a seguinte relação de comutação:

$$
\left[b_{k}(t), b_{j}^{\dagger}(t)\right]=\delta_{k j}
$$

sendo $b_{i}$ o operador de destruição e $b_{i}^{\dagger}$ o operador de criação. Com esses operadores, criamos o sistema de muitos corpos:

$$
|\vec{n}, t\rangle=\frac{1}{\sqrt{\prod_{i=1}^{M} n_{i} !}} \times \prod_{i=1}^{M}\left[b_{i}(t)\right]^{n_{i}}|0\rangle
$$

sendo $\vec{n}=\left(n_{1}, \cdots, n_{M}\right)$ o vetor da ocupação de cada orbital com $\sum_{i=1}^{M} n_{i}=N$ e sendo $|0\rangle$ ० 
vácuo.

Para um sistema de muitos corpos, a função de onda é dada pela combinação linear das funções de onda de cada um deles:

$$
|\Psi(t)\rangle=\sum_{\vec{n}} C_{\vec{n}}|\vec{n}, t\rangle
$$

sendo a somatória sobre todas as configurações possíveis de $\vec{n}$ respeitando a restrição do número total de partículas. A partir disso pode-se deduzir as equações utilizando o formalismo lagrangeano e minimizando a seguinte ação:

$$
S\left(\left\{C_{\vec{n}}\right\},\left\{\varphi_{k}\right\}\right)=\int\left[\left\langle\Psi\left|H-i \frac{\partial}{\partial t}\right| \Psi\right\rangle-\sum_{k, j=1}^{M} \mu_{k j}(t)\left(\left\langle\varphi_{k} \mid \varphi_{j}\right\rangle-\delta_{k j}\right)\right] d t
$$

Como os coeficientes $\left\{C_{\vec{n}}\right\}$ e os orbitais $\left\{\varphi_{k}\right\}$ são independentes entre si, utiliza-se o método variacional com respeito a cada um deles separadamente. Dessa forma obtemos as equações da dinâmica dos coeficientes:

$$
i \dot{\mathbb{C}}(t)=\mathbb{H}(t) \mathbb{C}(t)
$$

sendo o vetor $\mathbb{C}(t)$ composto pelos coeficientes e a matriz $\mathbb{H}(t)$ a matriz do hamiltoniano.

E temos a dinâmica da função de onda dos orbitais:

$$
i\left|\dot{\varphi}_{j}\right\rangle=P\left[h\left|\varphi_{j}\right\rangle+\sum_{k, s, q, l=1}^{M}\{\rho(t)\}_{j k}^{-1} \rho_{k s q l} W_{s l}\left|\varphi_{q}\right\rangle\right]
$$

sendo $P=1-\sum_{j^{\prime}=1}^{M}\left|\varphi_{j^{\prime}}\right\rangle\left\langle\varphi_{j^{\prime}}\right|$ o operador projeção, $h=T+V$ o operador hamiltoniano sem o termo de interação, $\{\rho(t)\}_{j k}^{-1}=\left\langle\Psi\left|b_{j}^{\dagger} b_{k}\right| \Psi\right\rangle$ os elementos da matriz inversa da matriz densidade reduzida de um corpo, $\rho_{k s q l}=\left\langle\Psi\left|b_{k}^{\dagger} b_{s}^{\dagger} b_{q} b_{l}\right| \Psi\right\rangle$ os elementos de matriz da matriz densidade reduzida de dois corpos e $W_{s l}=U_{0} \delta\left(\vec{r}_{s}-\vec{r}_{l}\right)$ o operador de interação entre partículas.

Essa teoria descreve o sistema exato no limite em que $M \rightarrow \infty$, caso contrário a série é truncada, mas conforme (10), ela converge bem rápido para sistemas simples como um duplo poço de potencial. 
1 Introdução 


\section{Dinâmica do Condensado de Bose-Einstein contendo sólitons}

\subsection{Objetivos e motivações}

O recente desenvolvimento das técnicas experimentais para lidar com os átomos ultrafrios, como o grande controle da interação atômica, pela técnica da ressonância de Feshbach, assim como a dimensão do sistema, com a utilização do confinamento em potenciais ópticos, corroboraram para um maior controle tanto do condensado quanto do sóliton. Essa grande evolução dos experimentos, por si só, já é um grande incentivo para estudos teóricos envolvendo os sólitons. Mas além disso, existe a possibilidade de utilizarmos essa excitação não linear macroscópica do BEC, o dark-soliton, para prever a validade do regime de campo médio.

Nesse trabalho exploramos como a presença do sóliton afeta as propriedades do condensado de Bose-Einstein (BEC) unidimensional aprisionado em armadilha harmônica, como seus modos normais (modos coletivos) de oscilação. Essa proposta é interessante a medida que nos auxiliaria na interpretação dos nossos resultados experimentais. Na rota da produção do condensado turbulento observamos que, após aplicação de uma excitação externa com amplitude e em intervalo de tempo apropriados, o condensado passa a apresentar oscilações de posição e formato da nuvem, que nos remetem aos seus modos coletivos. Mas observamos também estruturas no seu perfil de densidade, as quais associamos à presença dos vórtices. Queremos estudar como esses vórtices afetariam os modos. Em contrapartida, a própria caracterização do estado turbulento por meio da distribuição de momentos dos átomos da nossa amostra acaba sendo afetada pela presença dos modos coletivos, portanto precisamos saber individualizar esses efeitos característicos da excitação do superfluido. 


\subsection{Sistema estudado}

Vamos simular e analisar aqui diversas situações utilizando programas desenvolvidos em $\mathrm{C}++$, a partir de programas em Fortran presentes na literatura,(11) e em xmds2.(12)

Inicialmente, para validar os programas desenvolvidos, vamos testá-los com sistemas já muito bem conhecidos e descritos na introdução desse trabalho, como o modo dipolar do BEC aprisionado e a dinâmica do sóliton em um BEC inicialmente estático aprisionado em armadilha harmônica. Em seguida estudaremos sistemas simples como a dinâmica de um sóliton em um BEC inicialmente estático aprisionado em uma armadilha com impureza (barreira baixa). Por último passaremos para sistemas mais complexos como esses mesmos sistemas com barreiras altas e BECs em movimento contendo sólitons sem e com barreiras.

Vamos agora para o sistema físico estudado, com cada equação presente nas simulações. Vamos começar pelas GPEs adimensionais dependente e independente do tempo utilizadas:

$$
\begin{gathered}
-\frac{1}{2} \frac{d^{2}}{d x^{2}} \psi_{B E C, 0}(x)+\frac{1}{2} \omega^{2} x^{2} \psi_{B E C, 0}(x)+G_{0}\left|\psi_{B E C, 0}(x)\right|^{2} \psi_{B E C, 0}(x)=\mu \psi_{B E C, 0}(x) \\
-\frac{1}{2} \frac{\partial^{2}}{\partial x^{2}} \psi(x, t)+\frac{1}{2} \omega^{2} x^{2} \psi(x, t)+G_{0}|\psi(x, t)|^{2} \psi(x, t)=i \frac{\partial}{\partial t} \psi(x, t)
\end{gathered}
$$

Inicialmente simulamos a equação independente do tempo a partir de um ansatz para obtermos o estado fundamental do BEC nos parâmetros da simulação $\left(\psi_{B E C, 0}\right)$. Em seguida preparamos o sistema a ser estudado a partir do reposicionamento desse estado fundamental, da função de onda do sóliton e do potencial externo (com ou sem barreira).

Para o sóliton, temos:

$$
\psi_{\text {soliton }}=\sqrt{1-u^{2}} \operatorname{tgh}\left(\frac{x-x_{0}}{\xi} \sqrt{1-u^{2}}\right)+i u
$$

com $u=\frac{v}{c}$ sendo a velocidade do sóliton, $x_{0}$ sendo a posição do sóliton e $\xi$ sendo um parâmetro chamado healing length proporcional à largura do sóliton.

Para o potencial harmônico de aprisionamento, temos:

$$
V_{\text {harm }}(x)=\frac{1}{2} \omega^{2} x^{2}
$$


E para a barreira, temos:

$$
V_{\text {barr }}(x)=h_{\text {barr }} \mu+\left[\frac{1}{2} \omega^{2}-\frac{h_{\text {barr }} \mu}{\left(r_{\text {barr }} R_{T F}\right)^{2}}\right] x^{2}
$$

sendo $h_{b a r r}$ a altura da barreira em unidades do potencial químico e $r_{b a r r}$ a semi-largura da barreira em unidades do RTF.

Finalmente o potencial externo final fica:

$$
V(x)=\max \left\{V_{\text {harm }}(x) ; V_{\text {barr }}(x)\right\}=\frac{1}{2} \omega^{2} x^{2}+h_{\text {barr }} \mu \max \left\{0 ; 1-\left(\frac{x}{r_{\text {barr }} R_{T F}}\right)^{2}\right\}
$$

Aqui tem que ficar claro que o potencial químico tem que ser calculado considerando a barreira (apêndice E):

$$
\mu=\frac{1}{2}\left(\frac{3}{2} \frac{\omega G_{0}}{1-h_{\text {barr }} r_{\text {barr }}}\right)^{2 / 3}
$$

Vamos agora nomear e descrever cada um dos parâmetros utilizados nos programas a fim de facilitar a leitura dos resultados e análises desse trabalho.

- $G_{0} \rightarrow$ Parâmetro de interação

Valor padrão: 500 (regime de TF)

- $\omega \rightarrow$ Frequência da armadilha

Valor padrão: 1 (Outras frequências em unidades da frequência da armadilha)

- $R=r_{\text {barr }} R_{T F} \rightarrow$ Semi-largura da barreira em unidades do RTF Valor padrão: $0,05 R_{T F}$ (barreira fina)

- $H=h_{b a r r} \mu \rightarrow$ Altura da barreira em unidades do potencial químico

- $x_{C M} \rightarrow$ Posição inicial do centro de massa do BEC

Valor padrão: 3 para o sistema deslocado

- $x_{0} \rightarrow$ Posição inicial do sóliton em relação ao $C M$ em unidades do RTF Valor padrão: 0 (Centro do BEC)

- $u \rightarrow$ Velocidade do sóliton em unidades da velocidade do som Valor padrão: 0.5 para grey solitons e 0 para black solitons 
Para a análise dos dados, utilizando o software Matlab. Calculamos o centro de massa em função do tempo a partir de sua definição para uma função de onda normalizada:

$$
x_{C M}(t)=\int x|\psi(x, t)|^{2} d x
$$

Para o raio do condensado, utilizamos a semi-largura máxima da densidade, calculada a partir da busca por zeros de densidade depois de filtrada para a retirada de oscilações de alta frequência devido à presença do sóliton e das ondas sonoras.

A posição do sóliton é a mais difícil de ser encontrada, pois suas características se assemelham muito com outras posições do condensado quando a presença de ondas sonoras é muito intensa. Essa medida foi feita considerando os maiores picos da diferença entre o perfil de densidade na ausência e na presença do sóliton:

$$
\Delta \psi=\psi_{\text {ausência }}-\psi_{\text {presença }}
$$

Em posse das posições dos maiores picos da diferença $\Delta \psi$, analisamos os mais próximos à posição anterior do sóliton em relação à diferença de fase no core do sóliton estar próxima a $\pi$, definindo assim sua posição. Mesmo com esse extenso processo, veremos que esse resultado nem sempre é exato devido ao surgimento de outras excitações no sistema, que podem ser confundidas com o sóliton procurado. Para amenizar essas situações, depois de obtidas as curvas da posição do sóliton em função do tempo, passamos um filtro passa-baixa, eliminando as frequências muito maiores que a frequência de aprisionamento. Para finalmente obtermos os dados necessários às análises do nosso sistema. 


\section{Simulações e Resultados}

Neste capítulo, serão mostrados os resultados das simulações e suas análises. Teremos um tópico para cada simulação, iniciando pela simulação em potencial harmônico, que será posteriormente acrescido com uma barreira potencial e finalmente estudaremos o potencial harmônico com ranhuras.

\subsection{Sistema harmonicamente aprisionado}

Começaremos aqui com um potencial puramente harmônico, isto é:

$$
V(x)=\frac{1}{2} \omega^{2} x^{2}
$$

Com esse potencial de aprisionamento, estudaremos a dinâmica de um condensado puro em modo dipolar e na presença de um sóliton nos casos em que o BEC está inicialmente centrado na armadilha e em que o sistema está inicialmente deslocado.

\subsubsection{Condensado puro (modo dipolar)}

Apenas para validar a simulação, vamos considerar um condensado puro no estado fundamental deslocado de $x_{C M}$ do centro da armadilha. A figura 3.1 ilustra qualitativamente o movimento (modo dipolar) desse sistema em função do tempo. Essa imagem é a evolução temporal (horizontal) da densidade do BEC, como uma foto com exposição contínua, em que todo o movimento do sistema é capturado em apenas uma imagem. 
Figura 3.1 - Imagem de um movimento genérico de um BEC deslocado do centro da armadilha

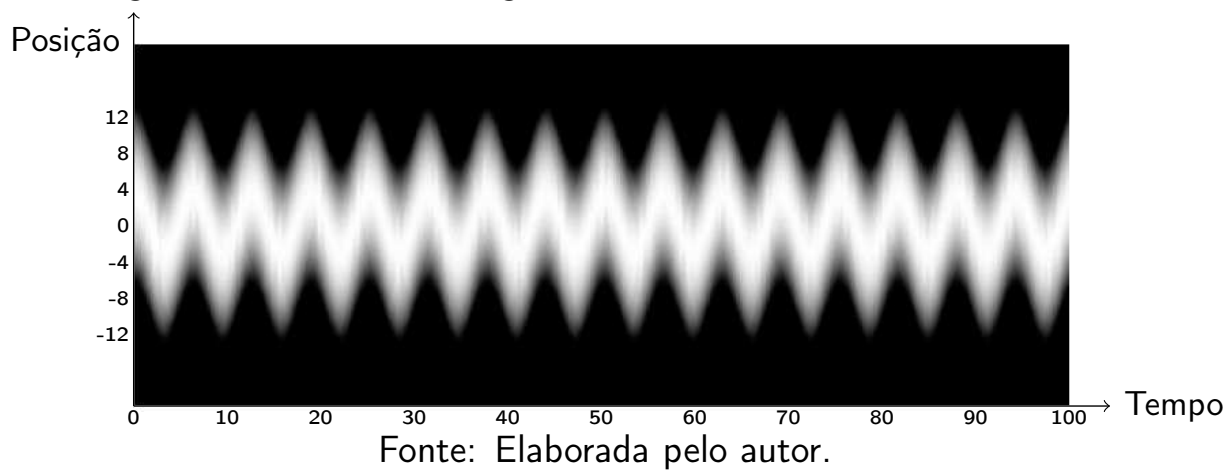

Somente para ilustrar e explicar melhor, a imagem 3.2 mostra a evolução temporal relativa ao primeiro período do movimento mostrado na figura 3.1. que é o tipo das figuras que mostraremos nas próximas seções desse trabalho.

Figura 3.2 - Evolução temporal do primeiro período $(T)$ da função de onda relativa à figura 3.1

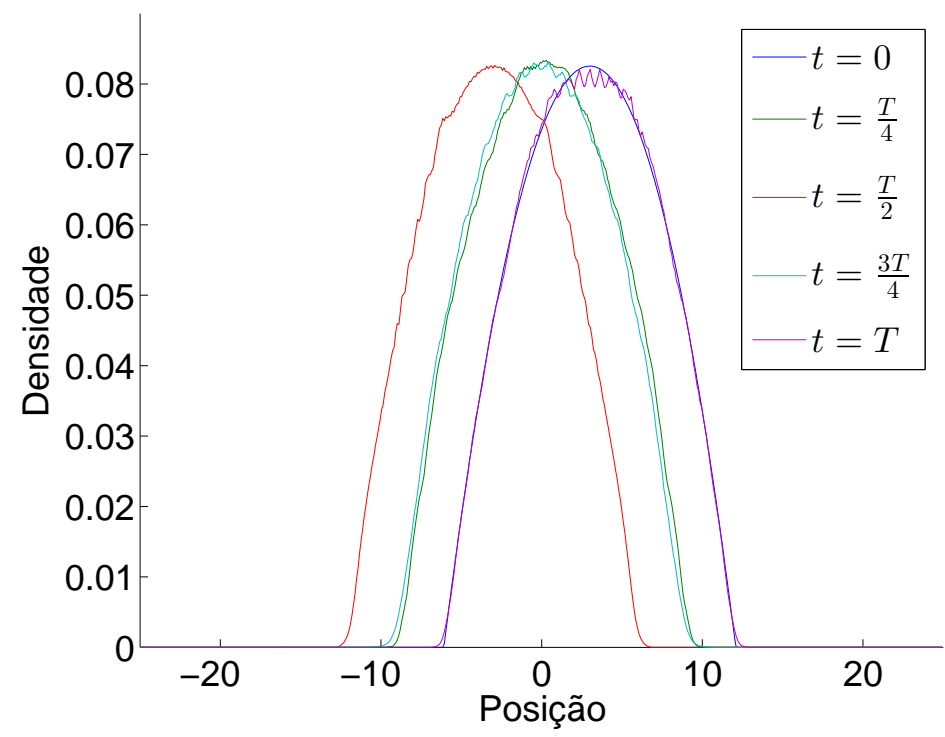

Fonte: Elaborada pelo autor.

Podemos observar uma oscilação de amplitude constante. Espera-se que a frequência desse movimento observado seja $\omega$, igual à frequência da armadilha (apêndice $B$ ). Faremos algumas simulações com potenciais harmônicos diferentes para evidenciarmos o resultado. Vamos considerar para essas simulações os seguintes parâmetros: $G_{0}=500, \omega \in\{1 ; 2 ; 3\} \mathrm{e}$ $x_{C M}=3,0$. Dessa forma obtemos os gráficos mostrados nas figuras 3.3, 3.4 e 3.5 para as dinâmicas do CM. 
Figura 3.3 - Modo dipolar para $\omega=1$.
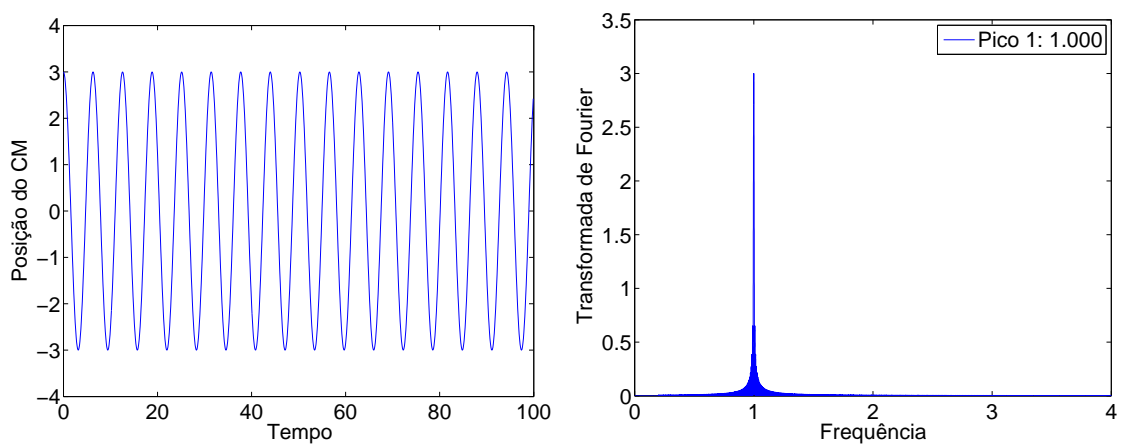

Fonte: Elaborada pelo autor.

Figura 3.4 - Modo dipolar para $\omega=2$.
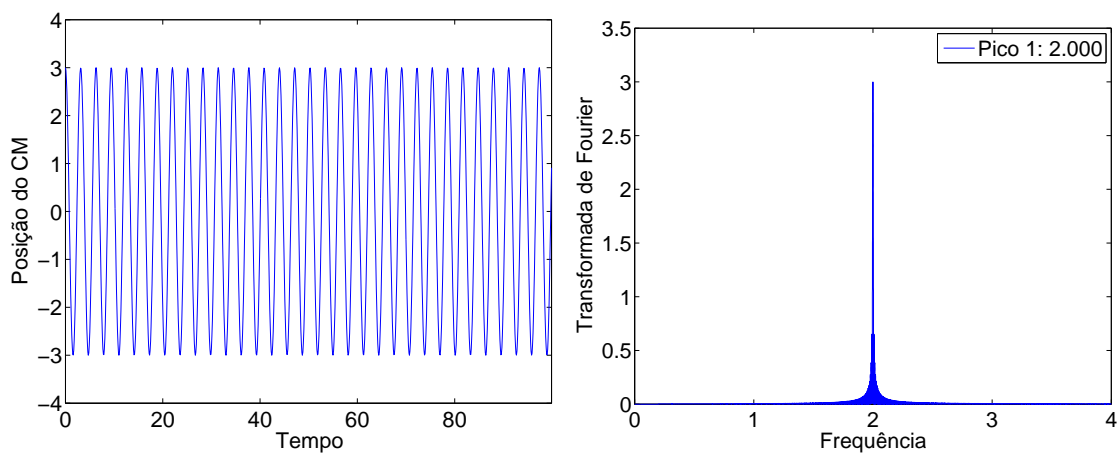

Fonte: Elaborada pelo autor.

Figura 3.5 - Modo dipolar para $\omega=3$.
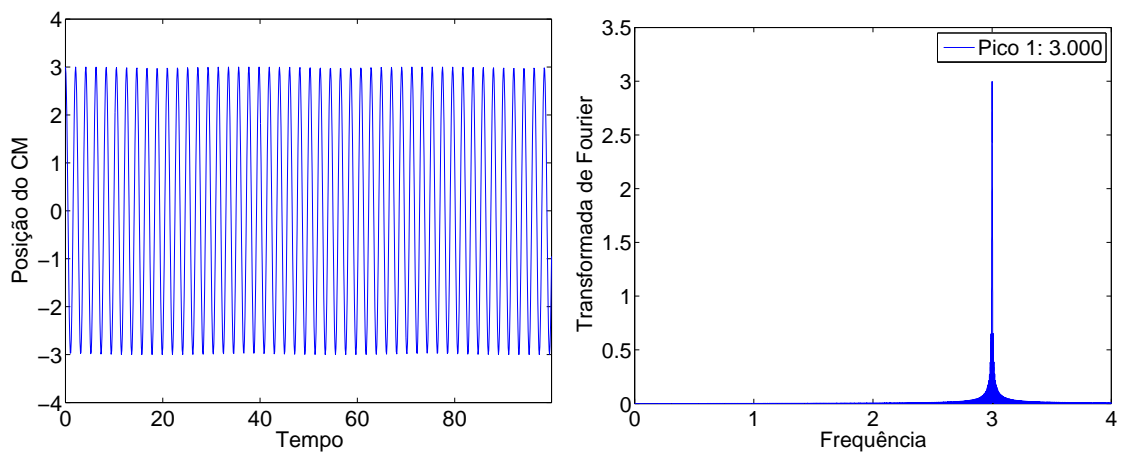

Fonte: Elaborada pelo autor.

Pode-se observar, através dos gráficos das Transformadas de Fourier, que as frequências dos movimentos do BEC são iguais às frequências das armadilhas como previsto previamente. 


\subsubsection{Condensado + Sóliton}

A seguir introduziremos um sóliton no BEC e analisaremos tanto o movimento do BEC quanto o movimento do sóliton. Este processo será feito em duas etapas: BEC inicialmente estático no centro da armadilha e BEC deslocado contendo um sóliton.

Iniciaremos com um BEC no estado fundamental no centro da armadilha contendo um sóliton (figura 3.6).

Figura 3.6 - Condição inicial do sistema BEC+grey soliton inicialmente centrado na armadilha

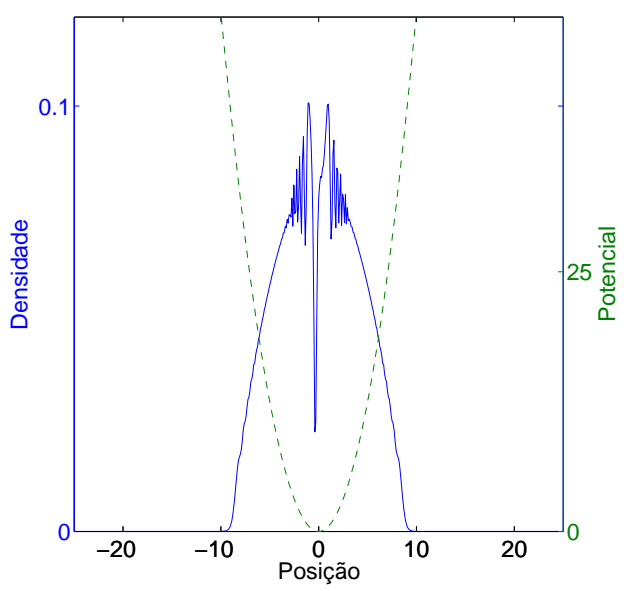

Fonte: Elaborada pelo autor.

Conforme estudamos, este sóliton deve se mover ao longo do BEC com uma frequência $\frac{\omega}{\sqrt{2}}$ emitindo ondas sonoras que podem perturbar o BEC. Vamos, então, primeiramente, aos parâmetros utilizados nessa simulação: $G_{0}=500, \omega=1, x_{C M}=0, x_{0}=0$ e $u=0,5$. Agora vamos à imagem qualitativa desse sistema (figura 3.7). 
Figura 3.7 - Imagem do movimento do grey soliton em um BEC inicialmente centrado na armadilha

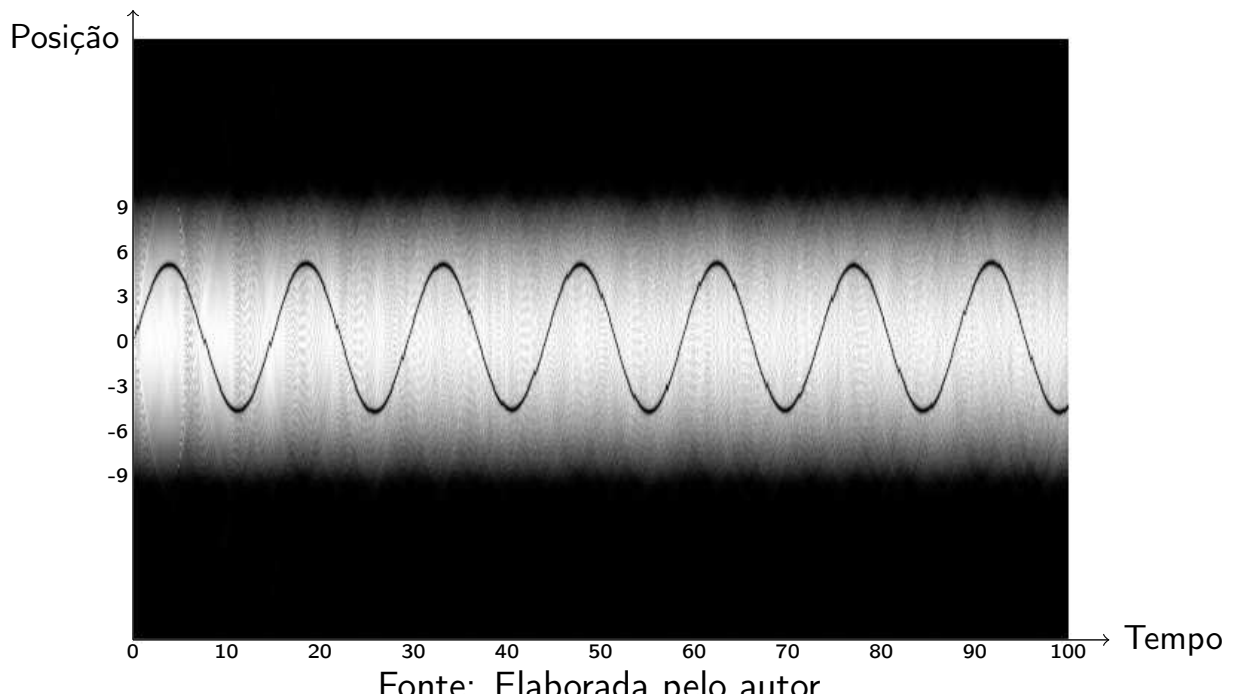

Fonte: Elaborada pelo autor.

Por essa imagem, já podemos tirar algumas características desse sistema, como a emissão de ondas sonoras e uma leve oscilação da posição do condensado. Vamos agora aos gráficos da dinâmica do sóliton para podermos analisar melhor essa situação (figura [3.8).

Figura 3.8 - Dinâmica do sóliton em um BEC inicialmente centrado na armadilha
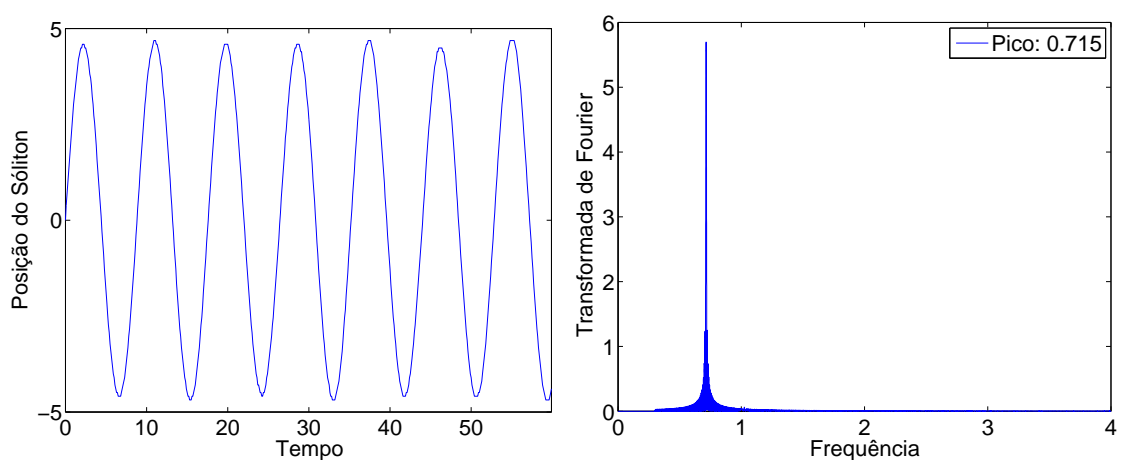

Fonte: Elaborada pelo autor.

A princípio, vamos validar a frequência do movimento. Como previsto, deveríamos ter uma frequência $\omega_{\text {sol }}=\frac{1}{\sqrt{2}} \approx 0,707$. O valor obtido é ligeiramente superior pois o cálculo dessa frequência admite o limite em que $2 G_{0} \gg 1$ (regime de TF). Valores maiores de $G_{0}$ iriam aproximar a frequência do valor calculado, mas diminuiriam outros efeitos que também gostaríamos de observar, como a perturbação do sistema devido às ondas sonoras.

A seguir temos uma característica importante proveniente do primeiro gráfico: os picos e vales do movimento não estão em uma posição constante, o que indica que há algum movimento do BEC que não previmos. Vamos então analisar a dinâmica do centro de massa 
do sistema (figura 3.9) para ter uma noção melhor do que está acontecendo.

Figura 3.9 - Dinâmica do CM do sistema BEC+grey soliton inicialmente centrado na armadilha
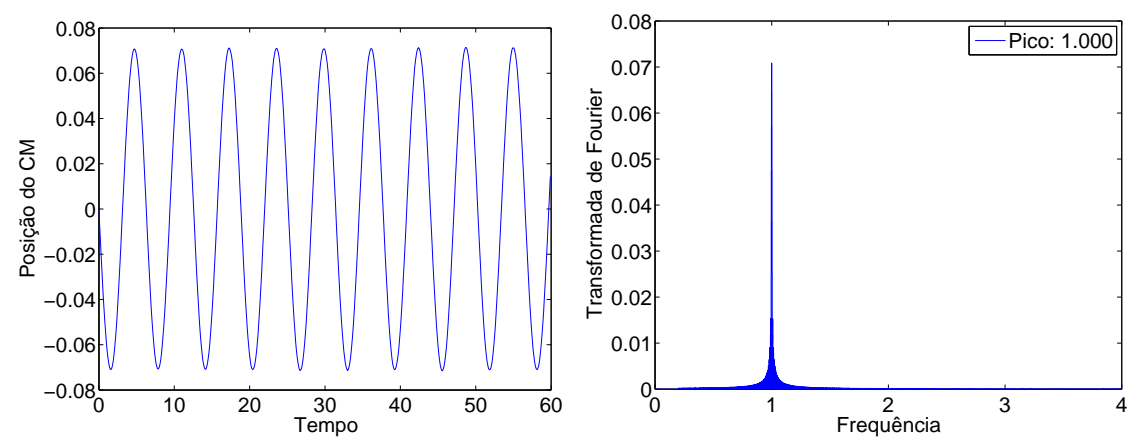

Fonte: Elaborada pelo autor.

Como estamos analisando a dinâmica do centro de massa do sistema (que inclui o BEC e o sóliton), poderia-se pensar que essa pequena oscilação ocorre devido ao movimento do sóliton, já que a amplitude do movimento é duas ordens de grandeza menor que o movimento do sóliton. Podemos provar que isso não é verdade pela frequência do movimento $\left(\omega_{C M}=\right.$ $\omega=\omega_{\text {dip }} \neq \omega_{\text {sol }}$ ), o que nos mostra que esse movimento do centro de massa é o modo dipolar do BEC excitado pelas ondas sonoras geradas pelo sóliton.

Mas esse é um grey soliton e portanto está em movimento desde o instante inicial gerando ondas sonoras assimetricamente a cada instante. Então vamos analisar um sistema semelhante mas com um black soliton. Agora teremos um sistema inicialmente simétrico (figura 3.10).

Figura 3.10 - Dinâmica do CM do sistema BEC+black soliton inicialmente centrado na armadilha
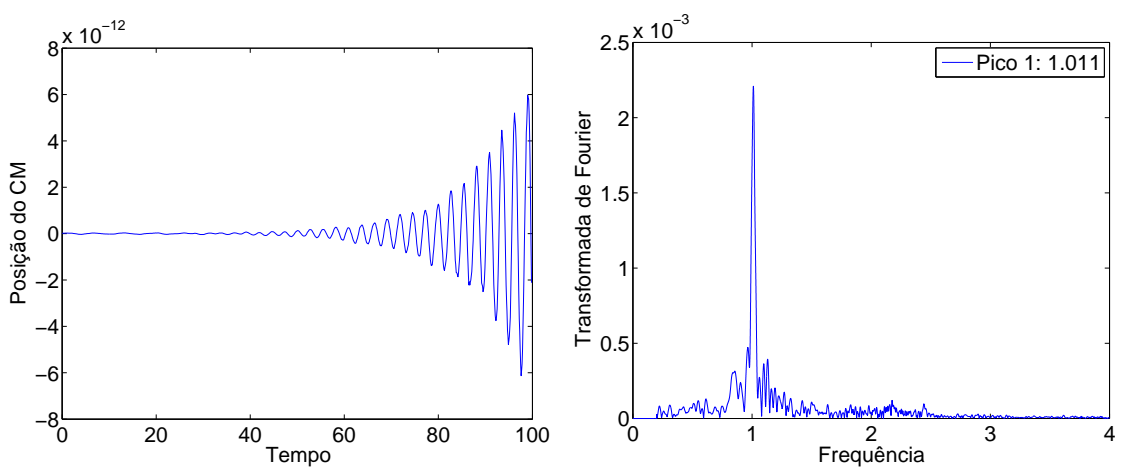

Fonte: Elaborada pelo autor.

Observe que devido à simetria, quase não há movimento do $\mathrm{CM}$, cuja amplitude é pouco crescente ao longo do tempo, porém o sistema é inicialmente simétrico, portanto há uma sutil quebra de simetria (da ordem de $10^{-11}$ ) que não deveria ocorrer pois não há nenhum agente 
externo que a cause. Esse deslocamento do centro de massa provavelmente é proveniente da discretização do sistema computacional, fato evidenciado pelo aumento da amplitude quando usamos precisão numérica inferior e diminuição da amplitude caso contrário. Depois do deslocamento inicial, observe que esse movimento é mesmo devido ao BEC (não ao sóliton) através da Transformada de Fourier.

A seguir vamos partir de um BEC no estado fundamental, adicionar um black soliton em seu centro, deslocar o sistema de $x_{C M}$ (figura ??) e verificar a dinâmica subsequente tanto do sóliton quanto do centro de massa do sistema.

Figura 3.11 - Condição inicial do sistema BEC+grey soliton inicialmente deslocado do centro da armadilha

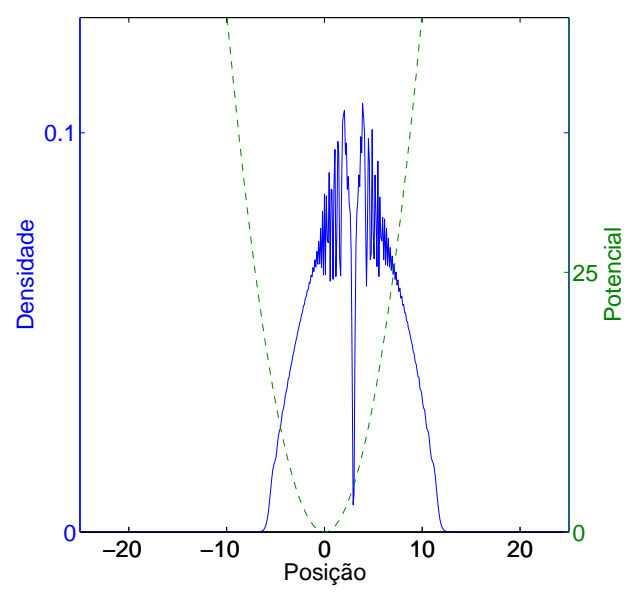

Fonte: Elaborada pelo autor.

Nessa simulação utilizamos os seguintes parâmetros: $G_{0}=500, \omega=1, x_{C M}=3,0$, $x_{0}=0$ e $u=0$. Agora vamos à imagem qualitativa desse sistema (figura 3.12).

Figura 3.12 - Imagem do movimento do sistema BEC+black soliton inicialmente deslocado do centro da armadilha

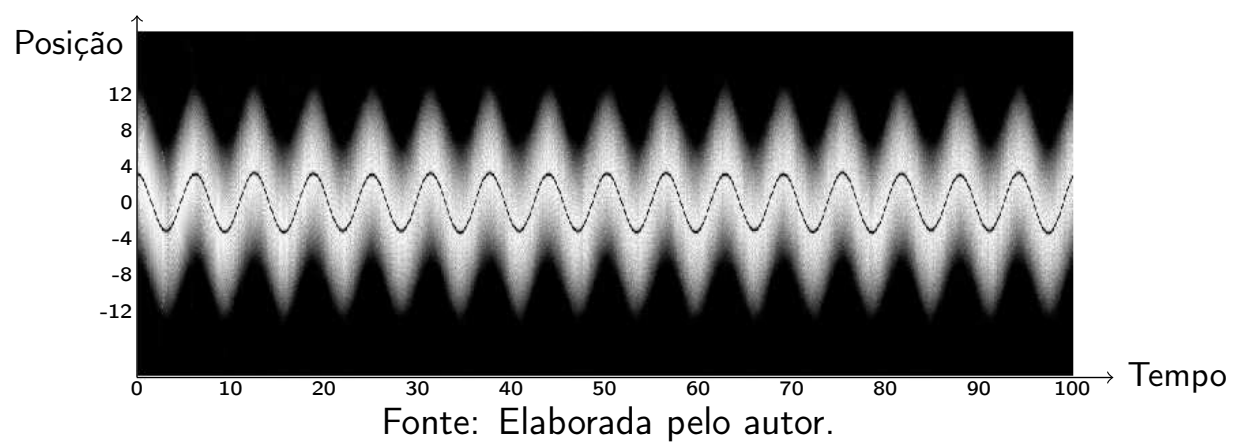

Observe um movimento praticamente em fase com o do BEC devido à pequena amplitude 
do movimento relativo entre o sóliton e o $\mathrm{BEC}$ frente à amplitude de movimento do $\mathrm{BEC}$ em relação à armadilha, o que faz o sóliton permanecer sempre bem próximo ao centro de massa do $\mathrm{BEC}$ e, portanto, se movimentar próximo à sua frequência, além do pico da frequência do sóliton se tornar desprezível frente ao pico do modo dipolar do BEC, o que pode ser verificado na figura 3.13 .

Figura 3.13 - Dinâmica de um black soliton em um BEC inicialmente deslocado do centro da armadilha
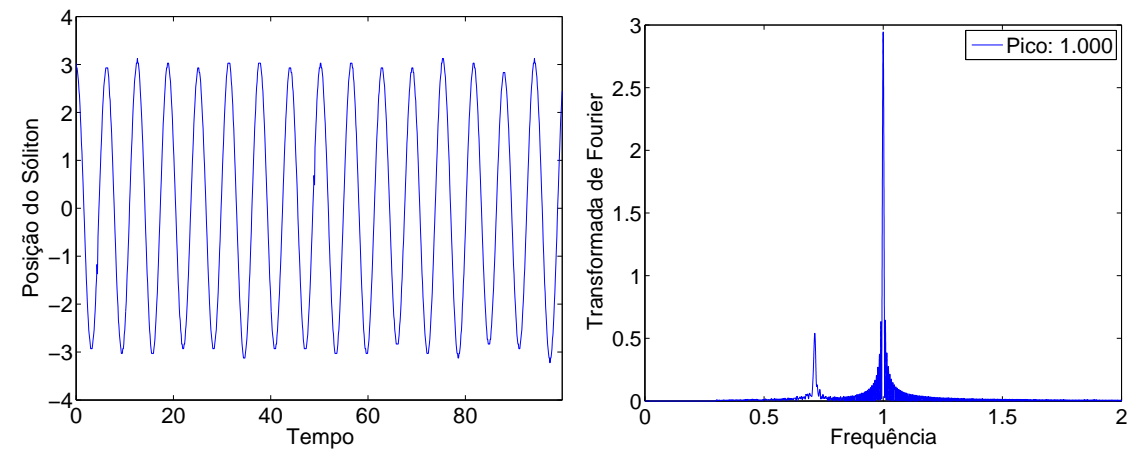

Fonte: Elaborada pelo autor.

Com o passar do tempo o sóliton perde energia em forma de ondas sonoras e, consequentemente, ganha amplitude de oscilação, fazendo com que a dinâmica se assemelhe mais à do caso com grey soliton. Isso fica evidente no pequeno pico no gráfico da Transformada de Fourier exatamente na frequência natural de oscilação do sóliton, já que com a perda de energia, a amplitude de oscilação do sóliton se torna visível.

Não vamos tratar o caso do BEC deslocado com um grey soliton, pois essa análise do sistema com black soliton já nos leva ao grey soliton depois de algum tempo devido à emissão de ondas sonoras, que reduz seu tamanho e aumenta sua velocidade, ou seja, o transforma em um grey soliton. As únicas diferenças em um grey soliton desde o início do movimento do BEC seriam um pico maior na transformada de Fourier relativo à frequência do sóliton e um movimento visualmente evidente na imagem qualitativa do movimento (figura 3.14). 
Figura 3.14 - Imagem do movimento do sistema BEC+grey soliton inicialmente deslocado do centro da armadilha

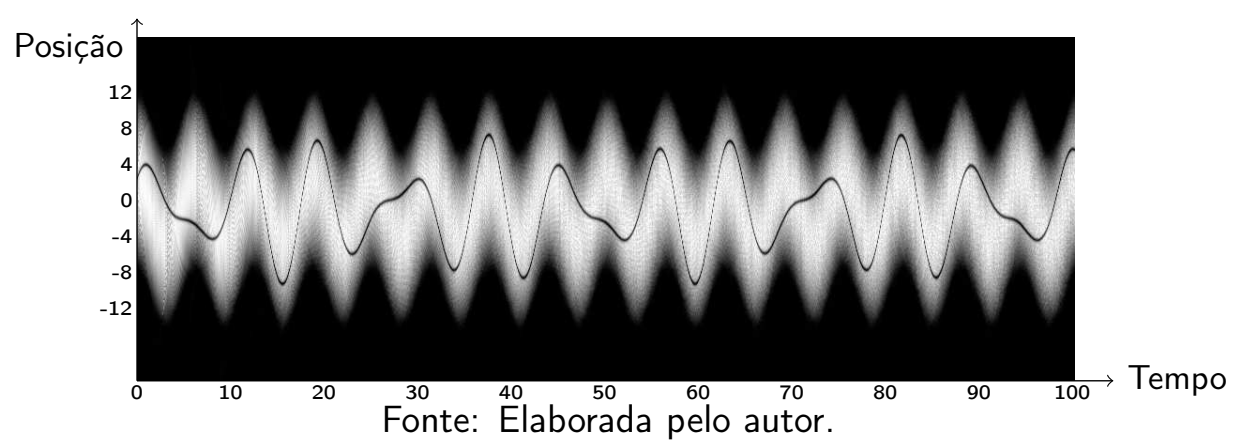

\subsection{Sistema harmonicamente aprisionado com barreira central}

Nessa seção, vamos adicionar uma barreira parabólica fina central, com a seguinte equação já explicada no capítulo 2.

$$
V(x)=\frac{1}{2} \omega^{2} x^{2}+h_{\text {barr }} \mu \max \left\{0 ; 1-\left(\frac{x}{r_{b a r r} R_{T F}}\right)^{2}\right\}
$$

de forma que a barreira tenha largura de $2 R=2 r_{b a r r} R_{T F}$, com $r_{b a r r}=0,05$ e altura $H=$ $h_{\text {barr }} \mu$, com $0 \leqslant h_{\text {barr }} \leqslant 5$.

\subsubsection{Condensado + Barreira}

Inicialmente vamos verificar as consequências da barreira no modo dipolar do BEC puro. Para essas simulações utilizaremos os seguintes parâmetros: $G_{0}=500, \omega=1, R=0,05 R_{T F}$, $H \in\{0,2 \mu ; 0,3 \mu ; 0,5 \mu ; 5 \mu\}$ e $x_{C M}=3,0$. Para esse primeiro caso (figura 3.15 ), utilizaremos uma barreira de altura $H=0,2 \mu$. 
Figura 3.15 - Condição inicial do BEC inicialmente deslocado do centro da armadilha com barreira baixa $(H=0,2 \mu)$

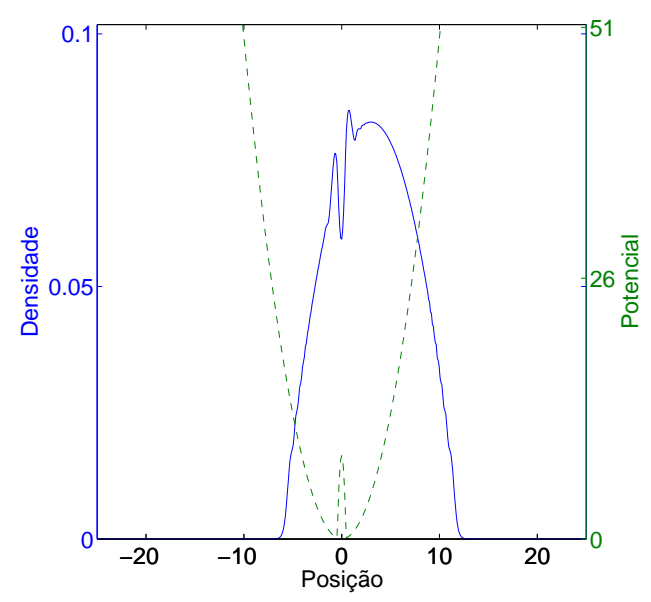

Fonte: Elaborada pelo autor.

A depressão observada na condição inicial do BEC é devido à repulsão causada pela barreira. A seguir (figura 3.16) vamos analisar a dinâmica decorrente desse estado inicial.

Figura 3.16 - Imagem do movimento de um BEC inicialmente deslocado do centro da armadilha com barreira baixa $(H=0,2 \mu)$

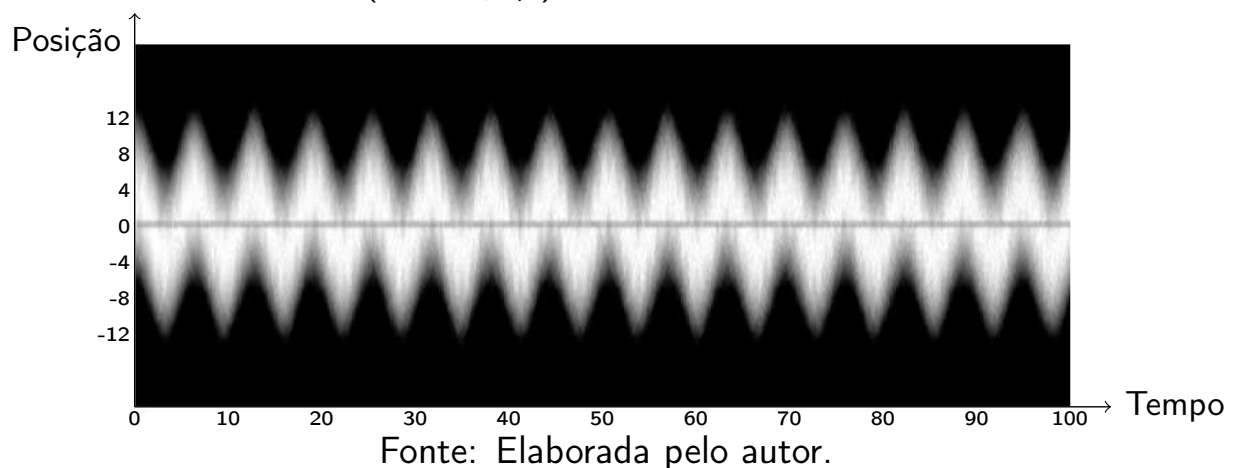

A reta horizontal verticalmente centralizada é uma depressão de densidade causada pela presença da barreira. Ao adicionarmos essa pequena barreira, nada aparentemente ocorre no tempo da simulação, o que não significa que não haveriam mudanças significativas se a simulação fosse executada por mais tempo. Vamos agora analisar na figura 3.17 como a presença da barreira afeta o movimento do centro de massa do sistema. 
Figura 3.17 - Dinâmica do BEC inicialmente deslocado do centro da armadilha com barreira baixa $(H=0,2 \mu)$
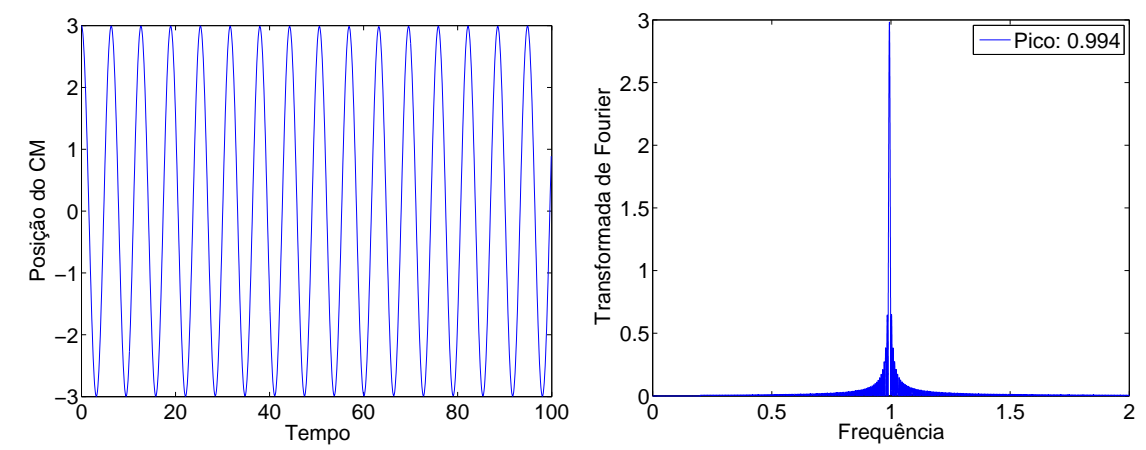

Fonte: Elaborada pelo autor.

Essa pequena barreira não afeta visivelmente o BEC no tempo estudado, mantendo tanto a dinâmica do CM quanto a Transformada de Fourier da mesma. A seguir vamos aumentar a barreira, criando o sistema cuja condição inicial é representada na figura 3.18 .

Figura 3.18 - Condição inicial do BEC inicialmente deslocado do centro da armadilha com barreira média $(H=0,3 \mu)$

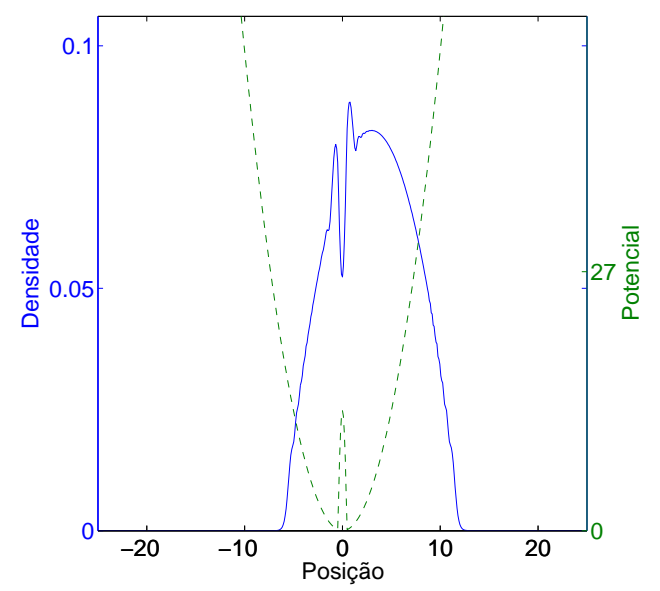

Fonte: Elaborada pelo autor.

Novamente a depressão devido à barreira aparece, maior agora devido ao aumento da altura da barreira. Ao utilizarmos essa barreira média, de altura $H=0,3 \mu$, algo importante pode ser observado já por análise qualitativa na figura 3.19. 
Figura 3.19 - Imagem do movimento do BEC inicialmente deslocado do centro da armadilha com barreira média $(H=0,3 \mu)$

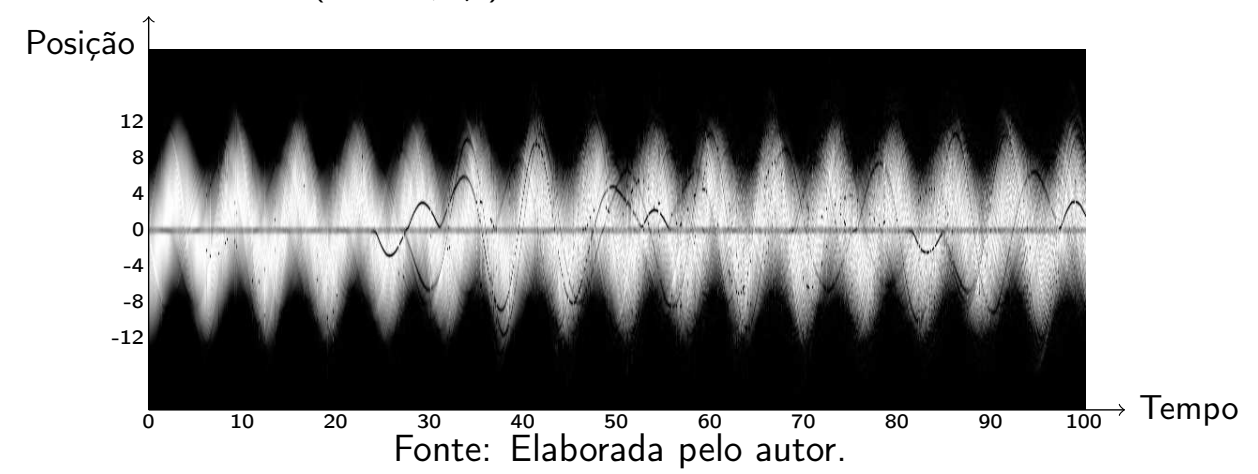

Essas depressões de densidade evidenciam fortemente a geração de sólitons na barreira.(13) As características que argumentam a favor de tal análise são: geração somente na barreira, transmissão e reflexão,(14-15) frequência natural do sóliton,(9) atração pela barreira,(16) barreiras menores não a geram, barreras maiores geram mais delas, ondas sonoras são visíveis na imagem, $(8,15,17)$ o que perturbam o sistema, e mais reflexões nas barreiras mais altas.

Já convencidos de que essas estruturas são sólitons, vamos agora aos gráficos do movimento desse BEC (figura 3.20).

Figura 3.20 - Dinâmica do BEC inicialmente deslocado do centro da armadilha com barreira média $(H=0,3 \mu)$
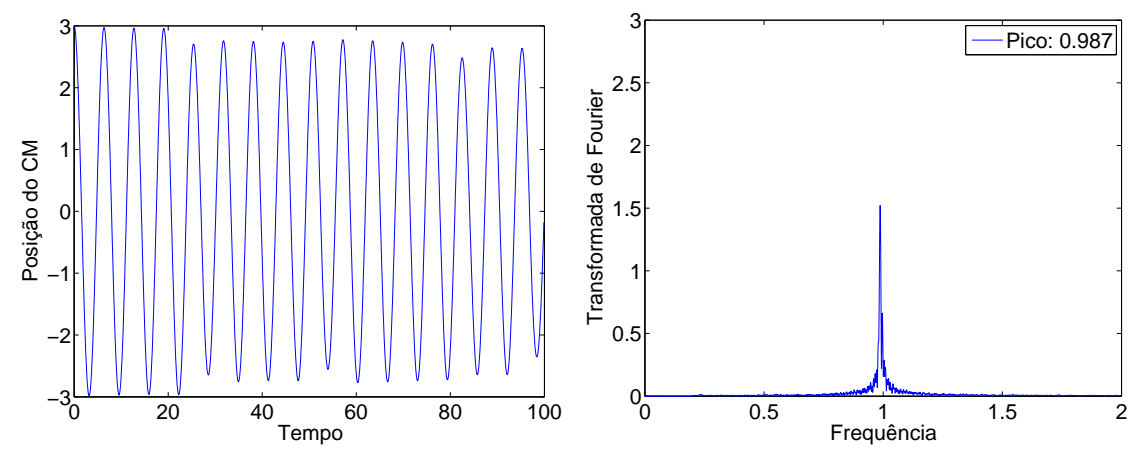

Fonte: Elaborada pelo autor.

Como já observado na imagem anterior, verifica-se perda de amplitude do sistema. Perceba que há uma queda brusca no quinto pico do movimento e que se voltarmos na figura 3.19. perceberemos que essa é justamente a região temporal em que surge o primeiro sóliton, ou seja, o que causa a perturbação de amplitude não é a barreira diretamente e sim o sóliton gerado por ela para o qual parte da energia do BEC tem que ser transferida para sua criação.

Vamos agora para a última simulação desse tipo de sistema, com barreira menor que a energia do condensado, com condição inicial dada pela figura 3.21 . 
Figura 3.21 - Condição inicial do BEC inicialmente deslocado do centro da armadilha com barreira alta $(H=0,5 \mu)$

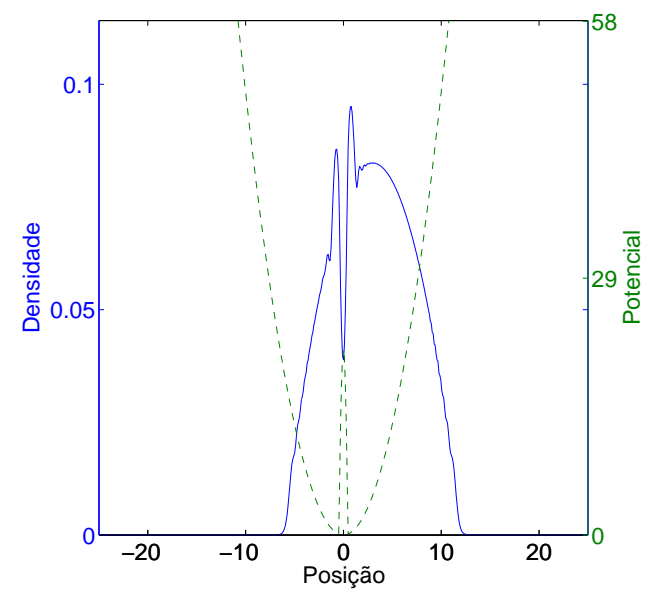

Fonte: Elaborada pelo autor.

Novamente observamos somente uma depressão inicial devido à barreira, maior que todos os casos anteriores devido ao aumento da altura da barreira. Vamos à dinâmica desse sistema (figura 3.22), apenas para reforçar os efeitos observados nos casos anteriores.

Figura 3.22 - Imagem do movimento de um BEC inicialmente deslocado do centro da armadilha com barreira alta $(H=0,5 \mu)$

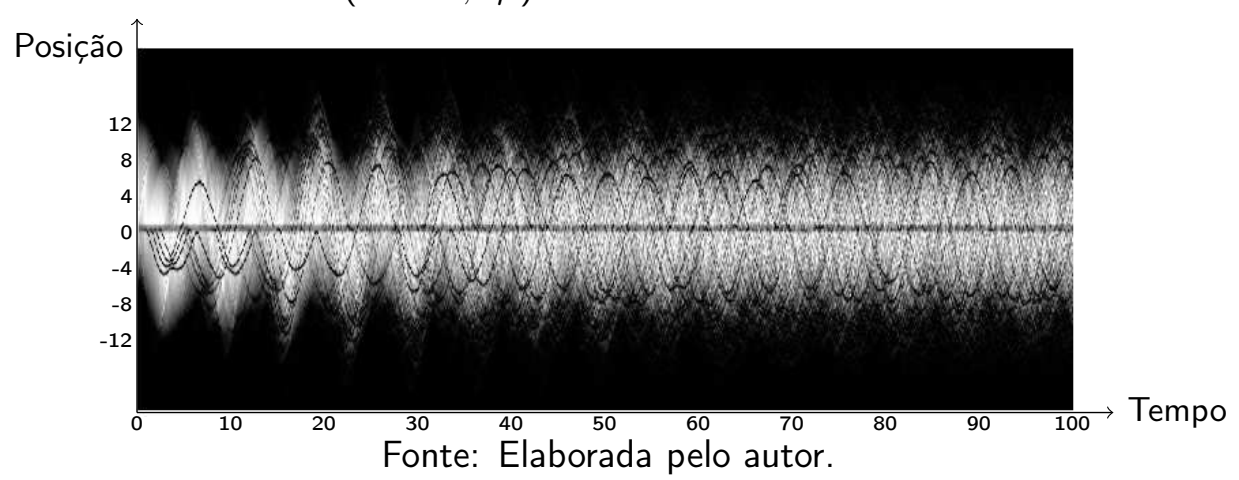


Figura 3.23 - Dinâmica do BEC inicialmente deslocado do centro da armadilha com barreira alta $(H=0,5 \mu)$
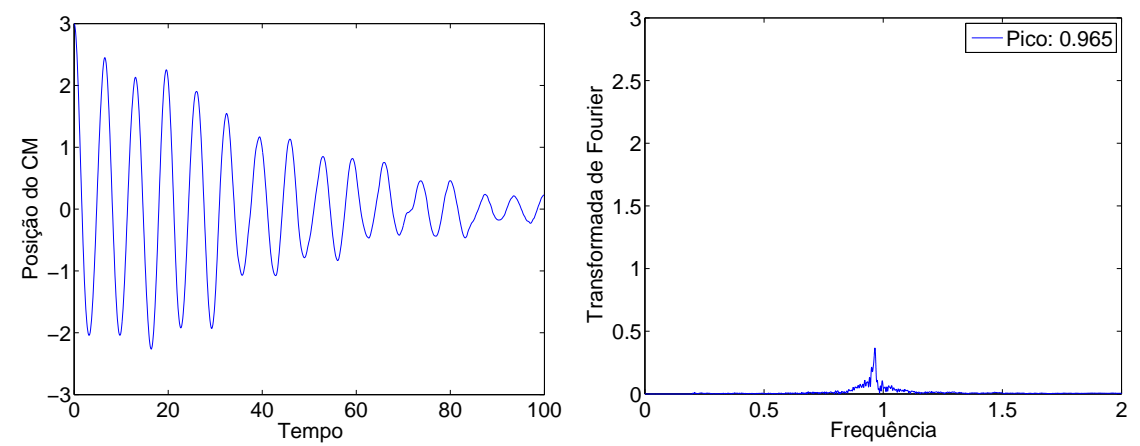

Fonte: Elaborada pelo autor.

Com a nova barreira observamos um número maior de sólitons nucleados e maior número de divisões do sóliton em transmissões e reflexões.

Vamos agora analisar o que acontece com o sistema quando utilizamos uma barreira bem maior que a energia do $\operatorname{BEC}(H=5 \mu)$, representado pela condição inicial mostrada na figura 3.24 .

Figura 3.24 - Condição inicial do BEC inicialmente deslocado do centro da armadilha com barreira bem maior que a energia do BEC $(H=5 \mu)$

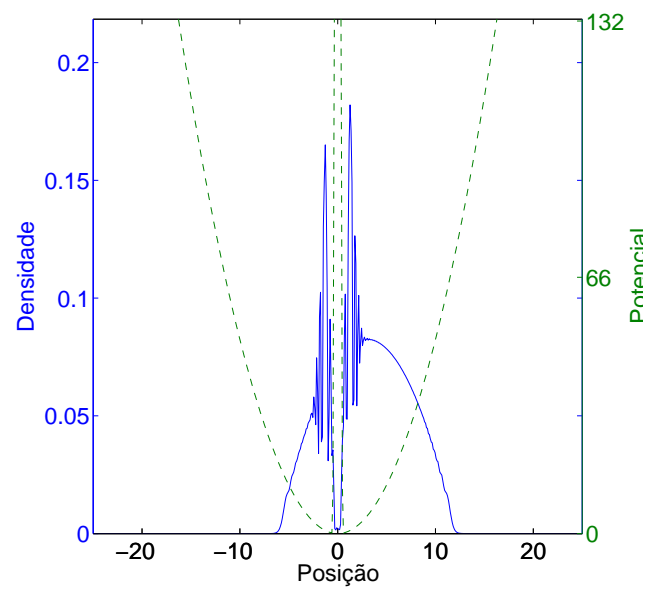

Fonte: Elaborada pelo autor.

Aqui vemos uma depressão bem maior, além de uma perturbação muito grande causada ao BEC pela adição da barreira. Vamos à imagem qualitativa da dinâmica do sistema na figura 3.25 
Figura 3.25 - Imagem do movimento do BEC inicialmente deslocado do centro da armadilha com barreira de altura $H=5 \mu$

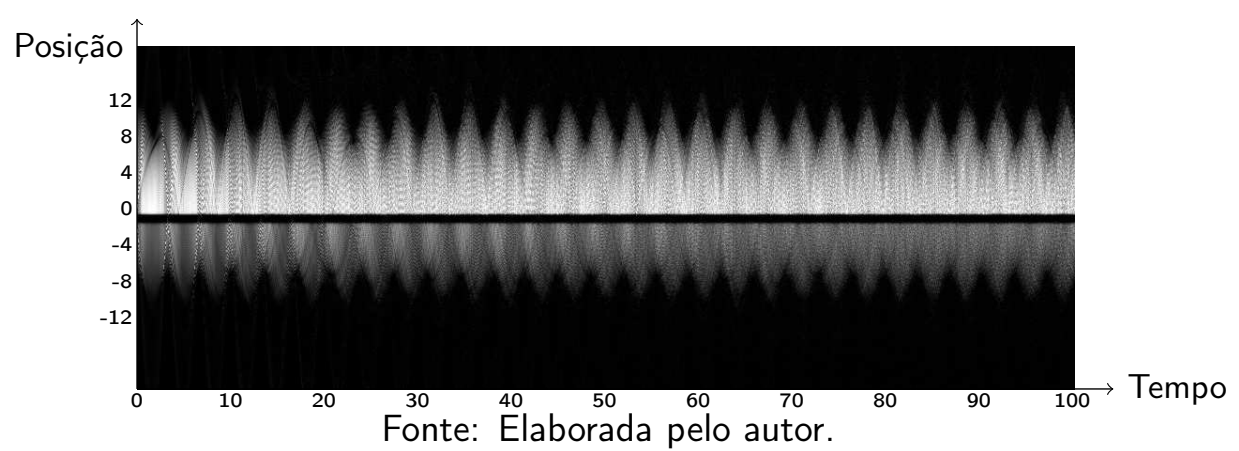

Observe que inicialmente o BEC começa a tentar oscilar no modo dipolar, mas como a barreira o impede e o mesmo possui uma energia inicial armazenada, a mesma é, na maioria, convertida em energia cinética para oscilação do raio do BEC, o que é evidenciado pela transformada de Fourier da dinâmica do raio e da dinâmica do CM. De acordo com a figura 3.26. os picos ocorrem um pouco abaixo de $2 \omega_{d i p}=2 \omega$ e um pouco acima de $\omega_{R}=\omega \sqrt{3}$, que são as frequências dos modos dipolar e breathing:

Figura 3.26 - Dinâmica do $\mathrm{CM}$ e do raio do $\mathrm{BEC}$, respectivamente, com $H=5 \mu$
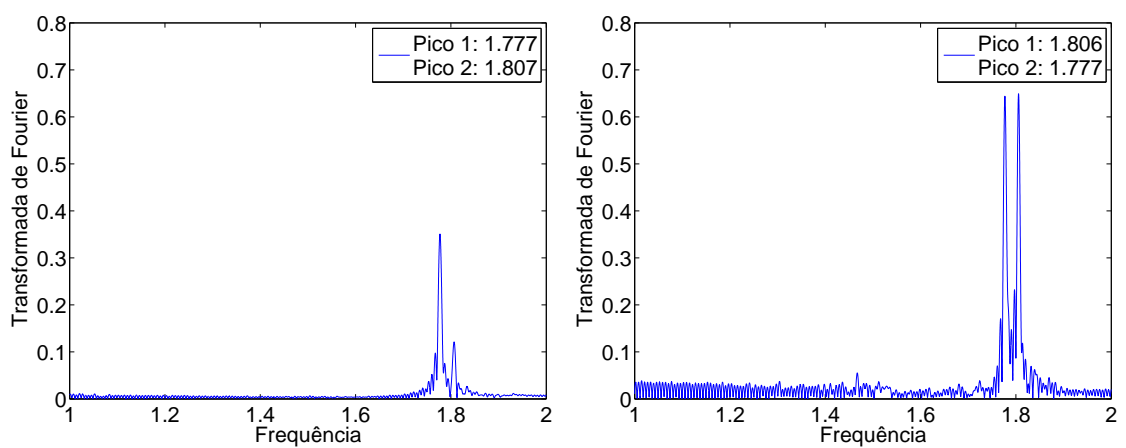

Fonte: Elaborada pelo autor.

A pequena alteração das frequências dos modos se devem à existência da barreira extremamente alta que interage muito com as partículas próximas fazendo com que haja uma leve diminuição da frequência do modo dipolar e um leve aumento na frequência do modo breathing. Os dois picos aparecem em ambas as transformadas pois a barreira cria uma forte ligação entre os dois modos, acoplando-os, já que, ao aprisionarmos a maior parte do BEC apenas de um lado da armadilha, oscilações do raio causam de forma direta oscilações no centro de massa e a oscilação do raio em parte é causada pela resposta da barreira à pressão que o sistema exerce na frequência de seu movimento, ou seja, na frequência do modo dipolar. 


\subsubsection{Condensado + Sóliton + Barreira Baixa}

Em relação ao sistema anterior, adicionaremos agora sólitons e estudaremos suas consequências para o sistema. Nessa seção trataremos de barreiras "baixas", tais que $0 \leqslant H \leqslant$ $0,3 \mu$.

Começaremos com o BEC inicialmente estático no centro da armadilha. Vamos escolher os parâmetros de forma que vejamos as consequências da inserção da barreira: $G_{0}=500$, $\omega=1, R=0,05 R_{T F}, H \in\{0,1 \mu ; 0,2 \mu ; 0,3 \mu\}, x_{C M}=0, x_{0}=0,4$ e $u=0,5$. As condições iniciais para os três sistemas são mostradas na figura 3.27 .

Figura 3.27 - Condições iniciais do sistema BEC+grey soliton inicialmente deslocado do centro da armadilha com barreiras de alturas $H \in\{0,1 \mu ; 0,2 \mu ; 0,3 \mu\}$
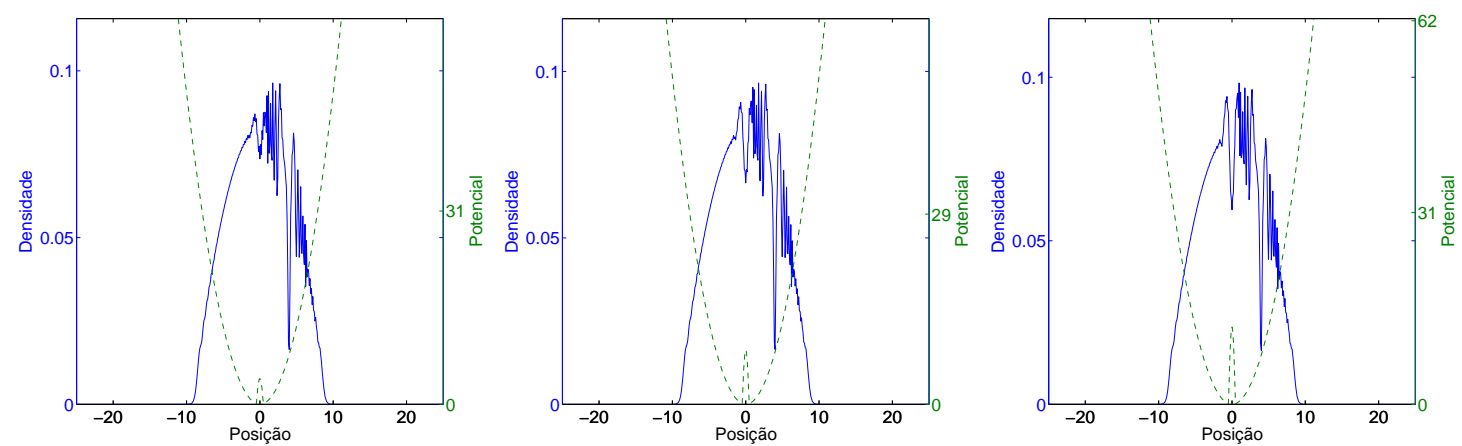

Fonte: Elaborada pelo autor.

Em todas elas, há duas depressões. A central é devido à barreira e a outra é o sóliton. Vamos às imagens qualitativas da dinâmica dos sisemas com as três alturas de barreiras (figura 3.28). 
Figura 3.28 - Imagem do movimento de um grey soliton em um BEC inicialmente centrado na armadilha com uma barreira de alturas $H \in\{0,1 \mu ; 0,2 \mu ; 0,3 \mu\}$, respectivamente

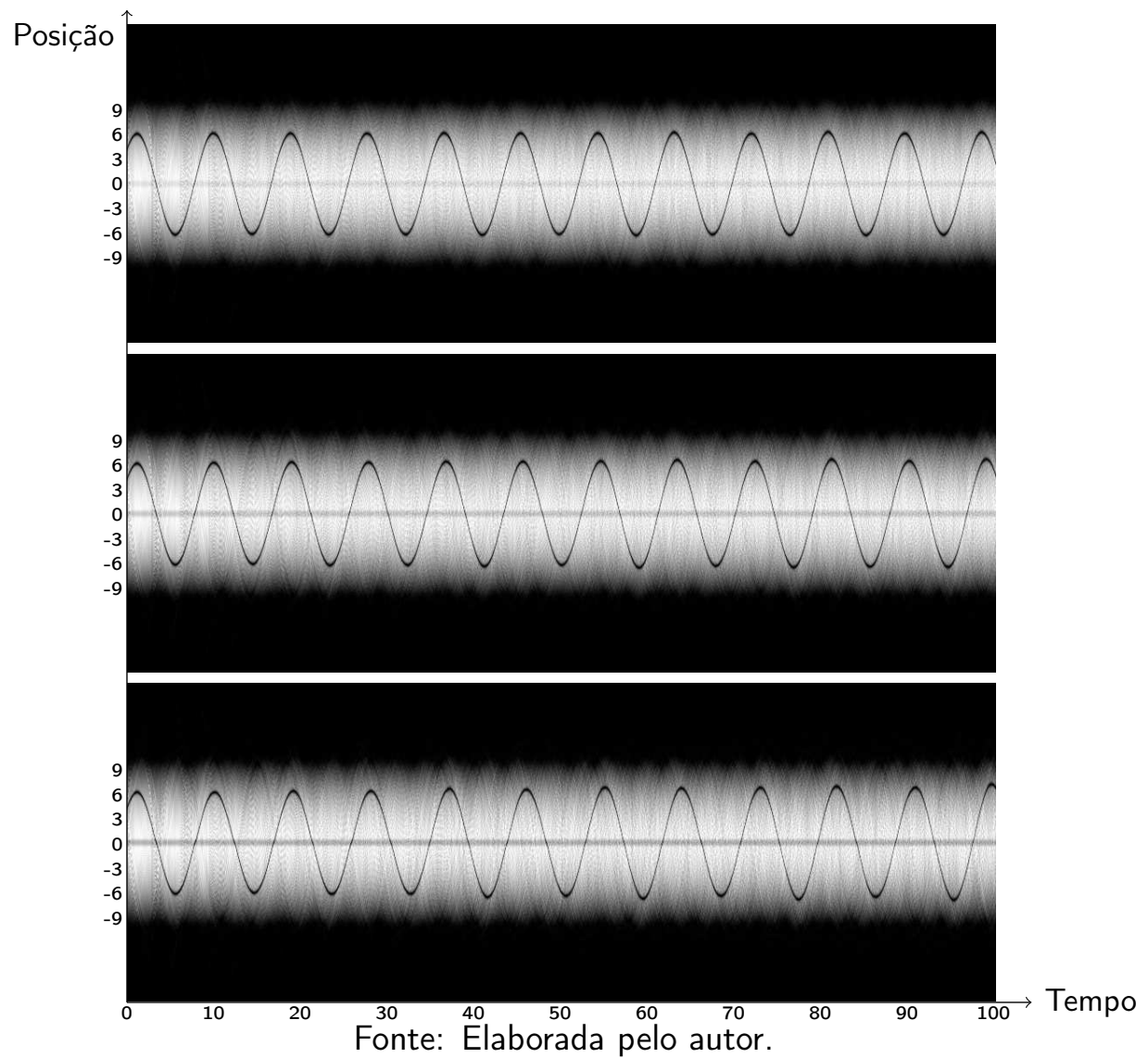

Nessas imagens, podemos ver a evolução da perturbação causada pelas ondas sonoras e da atração que a barreira causa no sóliton quando o mesmo a atravessa. Pode-se observar também o aumento dos picos de amplitude do sóliton para as barreiras mais altas, o que indica maior perda de energia. De fato, os gráficos de posição do sóliton em função do tempo (figura 3.29) mostram essa mesma conclusão.

Figura 3.29 - Dinâmica do grey soliton em um BEC inicialmente centrado na armadilha com barreiras de alturas $H \in\{0,1 \mu ; 0,2 \mu ; 0,3 \mu\}$, respectivamente
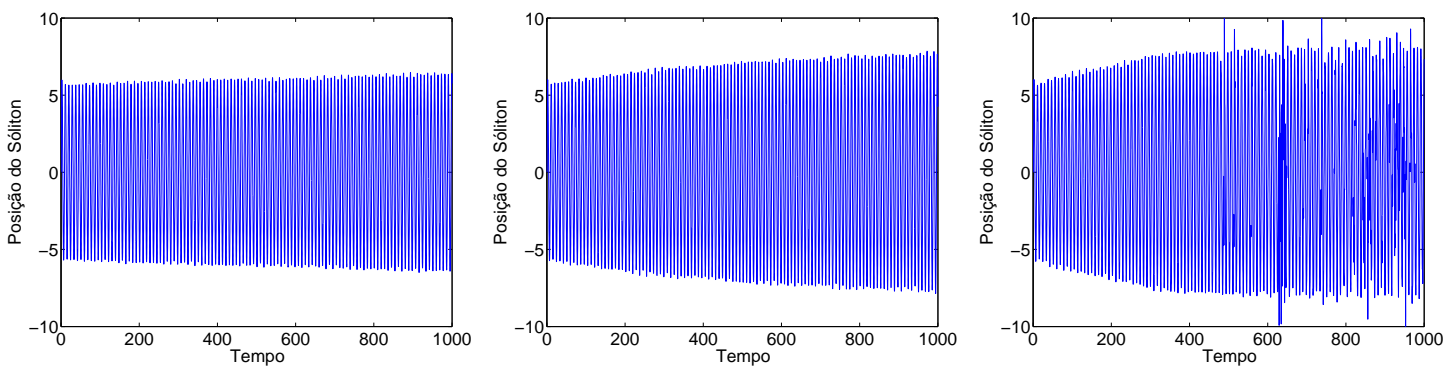

Fonte: Elaborada pelo autor. 
Observe que quanto maior a barreira, maior a perda de energia por unidade de tempo, caracterizada pela taxa de aumento da amplitude do movimento, que é maior quanto maior a altura da barreira. Perceba ainda que a energia (ou a amplitude de oscilação do sóliton) se estabiliza após certo tempo. Isso ocorre, pois após a perda de certa quantidade de energia o sóliton tem tamanho suficientemente pequeno para passar pela região da barreira sem tocar o zero de densidade e passa a perder energia muito mais vagarosamente, assim como se não houvesse barreira, afinal para ele, na verdade não há.

Se analisarmos agora a frequência desses três movimentos (figura 3.30), perceberemos que não há alteração na posição dos picos, afinal a frequência natural de oscilação do sóliton em um BEC harmonicamente aprisionado no regime de TF é a mesma, então não há razão para a mesma se alterar muito com o aumento de pequenas barreiras, já que o sóliton não toca o zero de densidade nesses casos. Porém, com barreiras mais altas, a taxa de emissão das ondas sonoras será maior.(8) Dessa forma é esperado que a largura do pico de frequência seja maior, representando essa perda de energia do sóliton, também refletida na sua maior amplitude de movimento e menor profundidade.

Figura 3.30 - Transformada de Fourier da dinâmica do grey soliton em um BEC inicialmente centrado na armadilha com barreiras de alturas $H \in\{0,1 \mu ; 0,2 \mu ; 0,3 \mu\}$, respectivamente
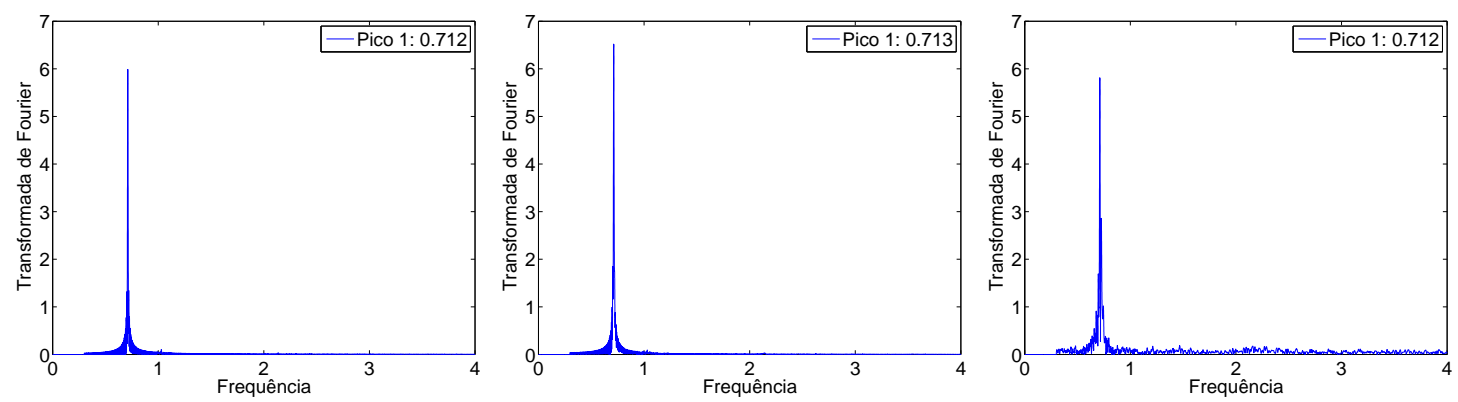

Fonte: Elaborada pelo autor.

Vamos analisar agora o quanto a barreira e, consequentemente, o aumento das ondas sonoras afeta no movimento do BEC analisando a posição do centro de massa em função do tempo (figura 3.31). 
Figura 3.31 - Dinâmica do CM do sistema BEC+grey soliton inicialmente centrado na armadilha com barreiras de alturas $H \in\{0,1 \mu ; 0,2 \mu ; 0,3 \mu\}$, respectivamente
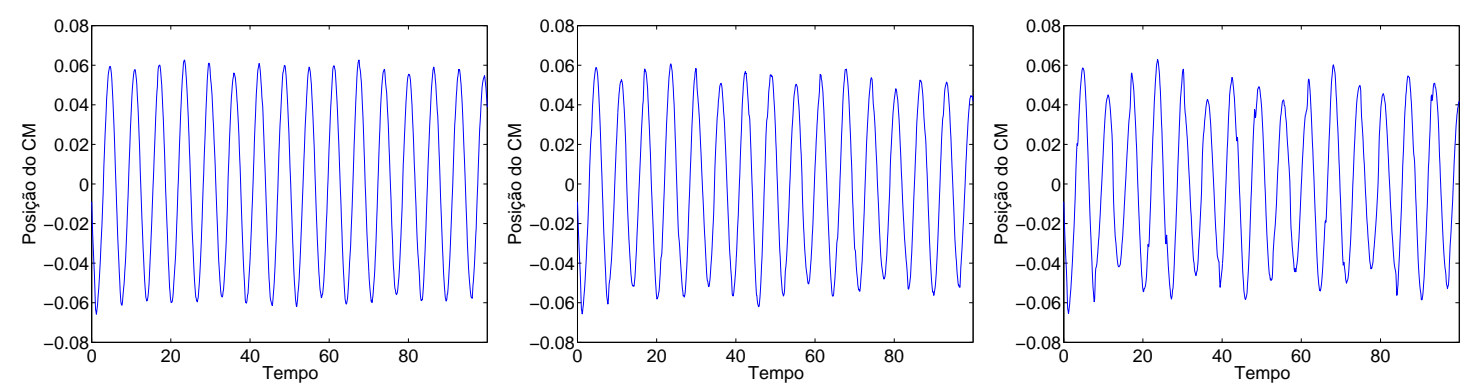

Fonte: Elaborada pelo autor.

Observe que quanto maior a barreira, maior a perturbação causada em relação ao sistema sem barreira, assim como na dinâmica do centro de massa do sistema sem sóliton. Mas observe também que há uma modulação do modo dipolar nesse gráfico. Vamos ver ao que ela se deve no gráfico da Transformada de Fourier (figura 3.32).

Figura 3.32 - Transformadas de Fourier da dinâmica do CM do sistema BEC+grey soliton inicialmente centrado na armadilha com barreiras de $H \in\{0,1 \mu ; 0,2 \mu ; 0,3 \mu\}$, respectivamente
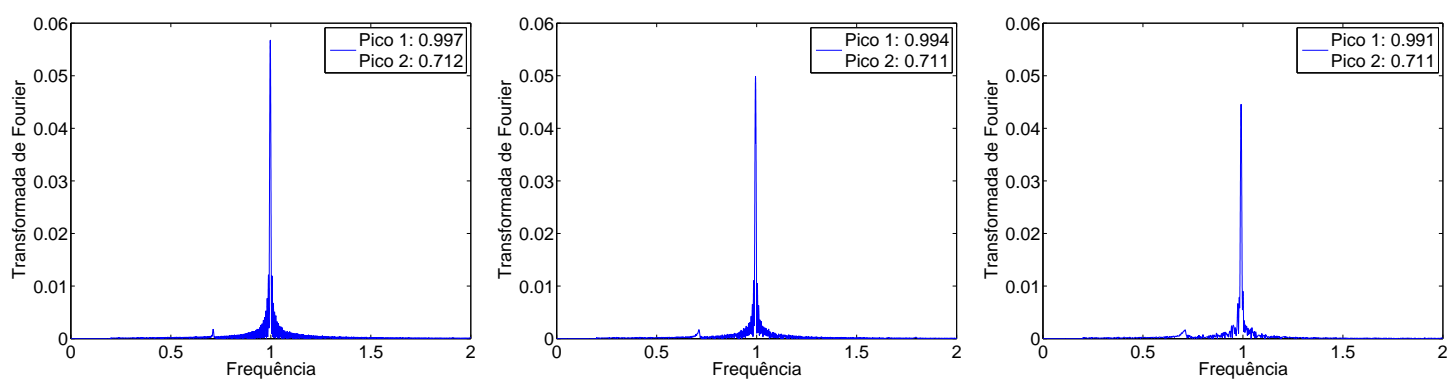

Fonte: Elaborada pelo autor.

Observe que há picos relativos ao movimento do $\operatorname{BEC}(1,00)$ mas também ao movimento do sóliton $(0,71)$, já que o deslocamento do sóliton faz com que haja um pequeno deslocamento do $\mathrm{CM}$.

Vamos agora utilizar um black soliton ao invés de um grey soliton e deslocar o sistema. Vamos começar com os parâmetros utilizados nessa simulação: $G_{0}=500, \omega=1, R=$ $0,05 R_{T F}, H \in\{0,2 \mu ; 0,3 \mu\}, x_{C M}=3,0, x_{0}=0$ e $u=0$. As condições iniciais para os três sistemas são mostradas na figura 3.33 . 
Figura 3.33 - Condições iniciais do sistema BEC+black soliton inicialmente deslocado do centro da armadilha com barreiras de alturas $H=0,2 \mu$ e $H=0,3 \mu$, respectivamente
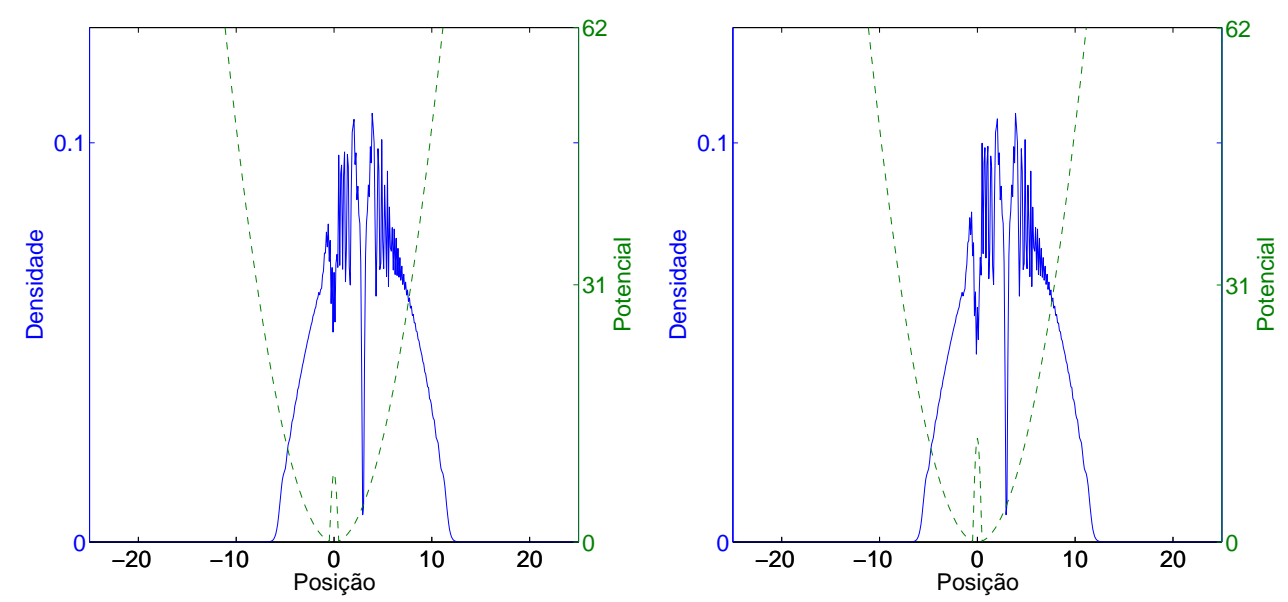

Fonte: Elaborada pelo autor.

Novamente há as mesmas duas depressões do sistema estudado anteriormente. Seguiremos agora, como na seção anterior, para a imagem qualitativa (figura 3.34).

Figura 3.34 - Imagens do movimento do sistema BEC+black soliton inicialmente deslocado do centro da armadilha com barreiras de alturas $H=0,2 \mu$ e $H=0,3 \mu$, respectivamente

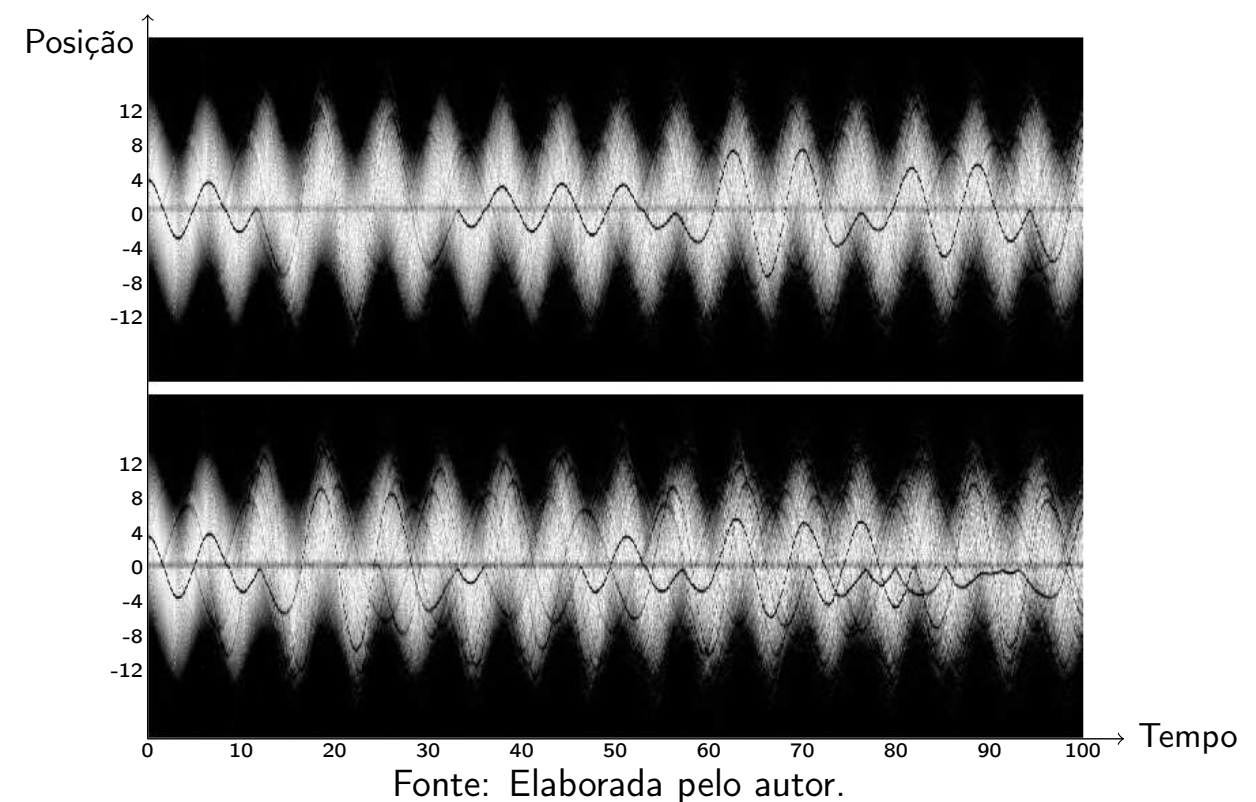

Observe que bem no início da imagem da barreira maior temos um comportamento semeIhante ao mesmo movimento sem barreira, exceto pelo fato de ocorrerem divisões do sóliton: parte é refletido, parte transmitido na barreira. Nesse caso pode-se pensar que na verdade não são divisões e sim a barreira gerando novos sólitons como já vimos anteriormente, mas observe que nessas casos, as supostas reflexão e transmissão formam ângulos que indicam fortemente 
esse fato, enquanto que nas gerações de sólitons pela barreira sempre há uma transição de velocidade muito suave, variando da velocidade nula até a velocidade de partida do sóliton gerado, como é possível observar em outras ocasiões nessa mesma imagem. A imagem da barreira menor mostra ora reflexão e ora transmissão do sóliton devido às trocas de energia em forma de ondas sonoras, que alteram a depressão do sóliton, ora permitindo sua transmissão, ora não. Observamos também a não geração de sólitons, fato importante para verificarmos o impacto somente do sólitons inicial no sistema, sem que haja geração de muitos sólitons que possam atrapalhar nossa análise.

Nessas mesmas imagens, ainda conseguimos observar emissão de ondas sonoras (perdas de energia), reabsorção de ondas sonoras (ganhos de energia) e repulsão entre sólitons próximos à barreira.

Vamos agora analisar o pouco que se consegue seguir do sóliton inicial (figura 3.35), afinal, pela figura acima pode-se imaginar a dificuldade disso, e ver o que obtemos com isso.

Figura 3.35 - Dinâmica do black soliton inicial do sistema BEC+black soliton inicialmente deslocado do centro da armadilha com barreiras de $H \in\{0,1 \mu ; 0,2 \mu ; 0,3 \mu\}$, respectivamente
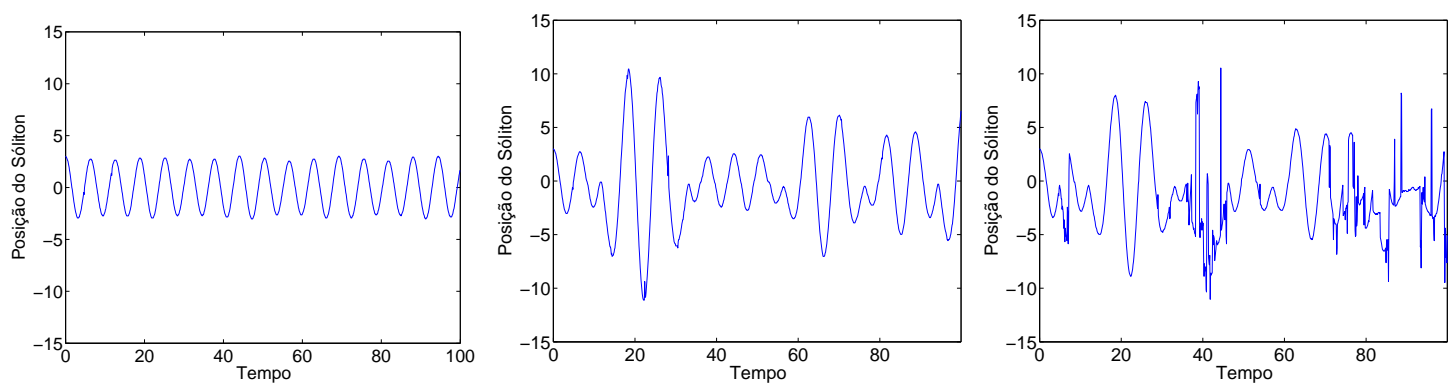

Fonte: Elaborada pelo autor.

Quanto maior a barreira, mais a dinâmica do sóliton é alterada em relação ao sistema sem barreira, isso porque há maior perda de energia, fazendo com que o sóliton comece a se mover mais rápido em relação ao $\mathrm{BEC}$ e mais novos sólitons são gerados, dificultando a perseguição do sóliton inicial. Como a perda de energia e, consequentemente, o aumento da velocidade, é mais rápida quanto maior a barreira, vejamos o que acontece se analisarmos a transformada de Fourier dessa dinâmica na figura 3.36 . 
Figura 3.36 - Transformada de Fourier dinâmica do black soliton inicial do sistema BEC+black soliton inicialmente deslocado do centro da armadilha com barreiras de $H \in$ $\{0,1 \mu ; 0,2 \mu ; 0,3 \mu\}$, respectivamente
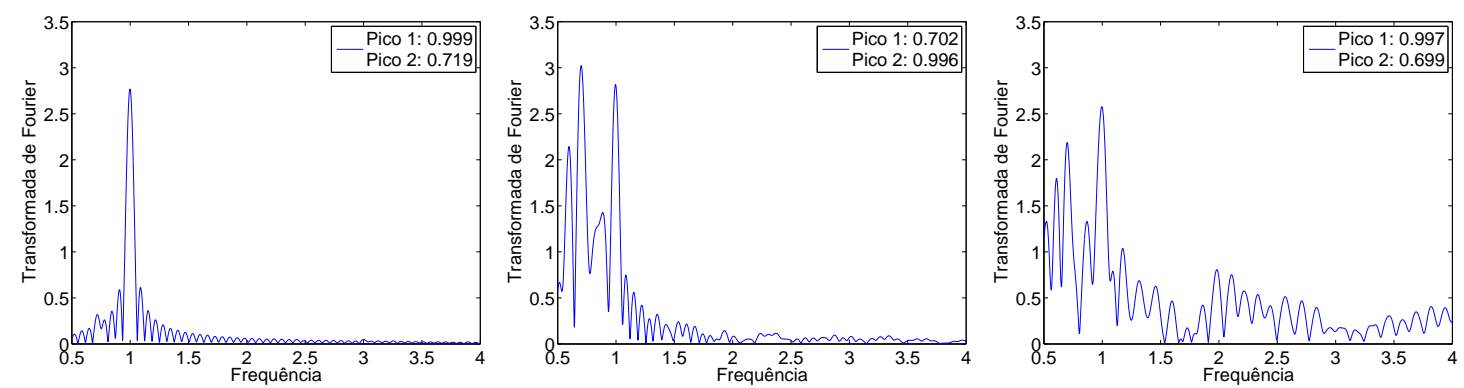

Fonte: Elaborada pelo autor.

Da segunda imagem para a terceira não há muita mudança, afinal a própria dinâmica já mostra que não há muita diferença. O que podemos observar claramente é a mudança do primeiro para o segundo gráfico. No primeiro o sóliton é praticamente somente arrastado pelo $B E C$, pois a perda de energia é muito lenta, fazendo com que apareça um pico muito alto do movimento do BEC e um pequeno do sóliton. Já no segundo, temos uma perda de energia muito rápida, transformando o black soliton em um grey soliton, fazendo o pico da frequência do sóliton aumentar consideravelmente, atingindo a mesma ordem de grandeza do BEC, ou seja, o movimento relativo passa a ser tão importante quanto o movimento do BEC em si. Vamos agora analisar o que a adição do sóliton muda em relação ao mesmo sistema sem sóliton através da análise da dinâmica do CM na figura 3.37 .

Figura 3.37 - Dinâmica do CM do sistema BEC+black soliton inicialmente deslocado do centro da armadilha com barreiras de $H \in\{0,1 \mu ; 0,2 \mu ; 0,3 \mu\}$, respectivamente
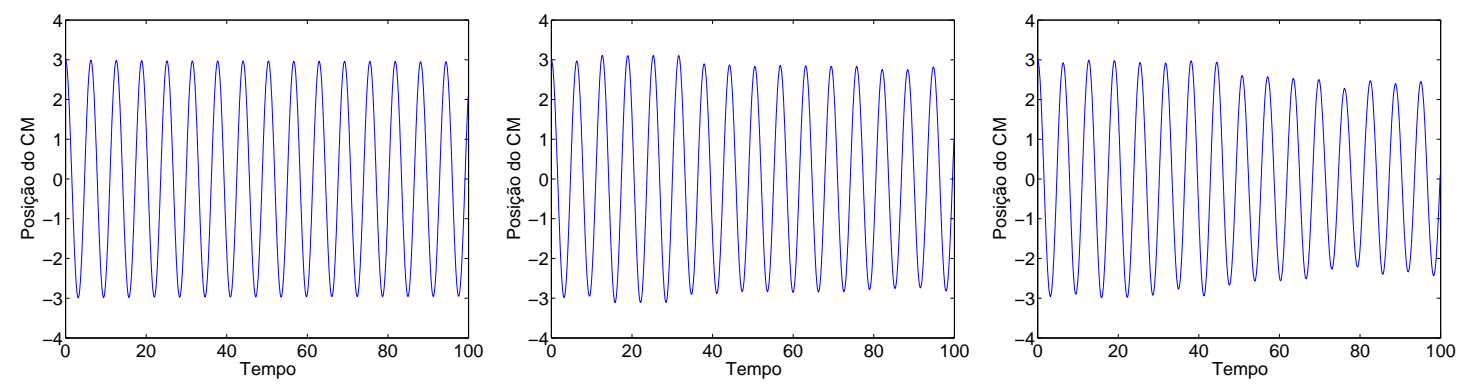

Fonte: Elaborada pelo autor.

Nesses gráficos, vemos o mesmo comportamento do sistema sem o sóliton inicial (troca de energia com os sólitons). Se compararmos esses gráficos com a figura 3.34, veremos que as mudanças bruscas de amplitude da dinâmica do centro de massa ocorrem quando há eventos relacionados ao sóliton, isto é, quando os sólitons recebem energia (geração de novos sólitons 
ou reabsorção de ondas sonoras) há perda de amplitude na dinâmica do CM e quando os sólitons cedem energia (emissão de ondas sonoras) há ganho de amplitude na dinâmica do CM. Se executarmos o mesmo programa por mais tempo, observamos, em média, uma perda de amplitude até uma estabilização, que ocorre devido à geração de muitos sólitons, o que causa perda de energia do BEC até o ponto em que a energia do BEC não é mais suficiente para gerar tantos sólitons e a amplitude estabiliza, sendo que a taxa dessa perda de energia é, em módulo, maior quanto maior for a barreira, já que, como visto anteriormente, quanto maior a barreira, maior a quantidade de sólitons gerados.

\subsubsection{Condensado + Sóliton + Barreira Alta}

Vamos agora estudar o sistema com uma barreira acima da energia do BEC. Ou seja, temos um BEC inicialmente em modo dipolar com um sóliton. Nessa seção utilizaremos $H=5 \mu$.

Vamos começar pelo black soliton com a condição inicial do sistema mostrada na figura 3.38 .

Figura 3.38 - Condição inicial do sistema BEC+black soliton inicialmente deslocado do centro da armadilha com barreira de altura $H=5 \mu$

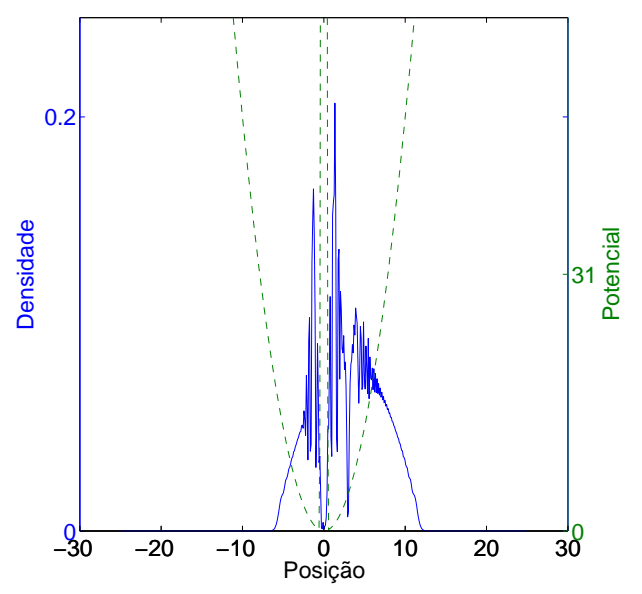

Fonte: Elaborada pelo autor.

Partindo dos resultados anteriores, devemos ter um sóliton aprisionado de um lado da barreira. Vamos começar com os parâmetros utilizados nessa simulação: $G_{0}=500, \omega=1$, $R=0,05 R_{T F}, H=5 \mu, x_{C M}=3,0, x_{0}=0$ e $u=0$. Seguiremos agora, como nas outras seções, para a imagem qualitativa (figura 3.39). 
Figura 3.39 - Imagem do movimento do sistema BEC+black soliton inicialmente deslocado do centro da armadilha com uma barreira de altura $H=5 \mu$

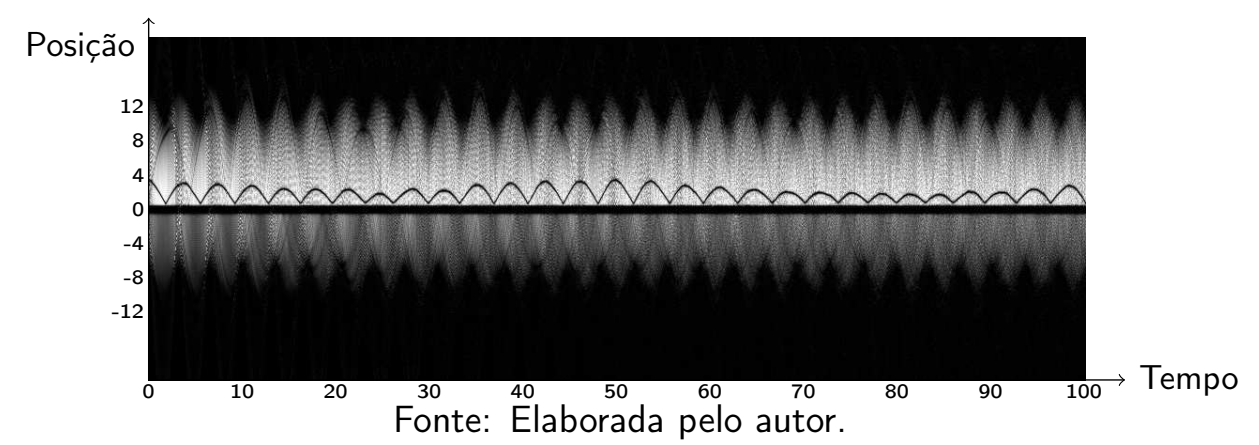

Assim como no caso do BEC sem sóliton, esse novo sistema não atravessa a barreira. A posição do sóliton em função do tempo está bem clara nessa imagem, possibilitando analisarmos somente a transformada de Fourier dessa dinâmica na figura 3.40 .

Figura 3.40 - Transformada de Fourier da dinâmica do black soliton inicial do sistema BEC+sóliton inicialmente deslocado do centro da armadilha com barreira de $H=5 \mu$

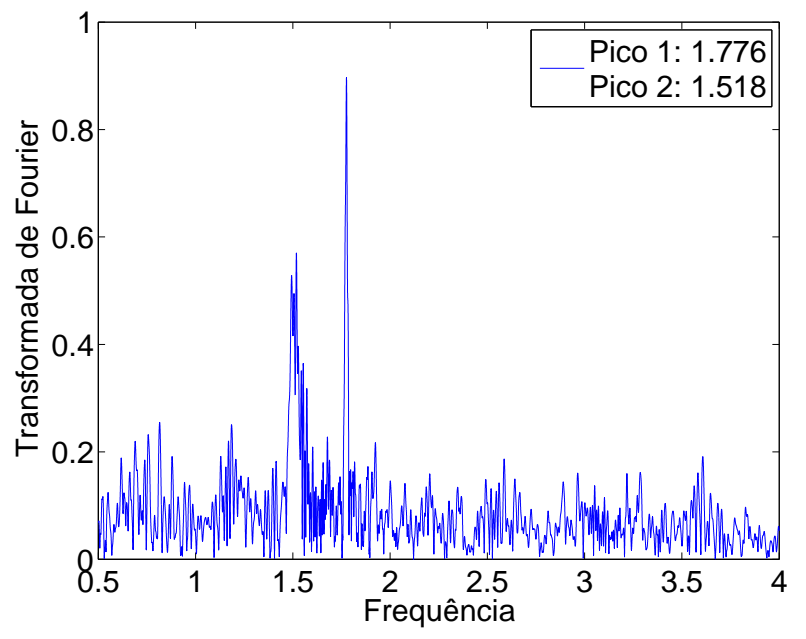

Fonte: Elaborada pelo autor.

Dentre o ruído, temos dois picos que se destacam, sendo um referente ao modo breathing $(1,776)$ e um referente referente à dinâmica do sóliton $(1,518)$, que é o dobro da frequência do sóliton, já que este reflete sequencialmente na barreira. Vamos agora à análise da transformada de Fourier da dinâmica do CM e do raio do BEC, respectivamente, na figura 3.41 . 
Figura 3.41 - Transformada de Fourier da dinâmica do CM e do raio do BEC, respectivamente, inicialmente deslocado do centro da armadilha
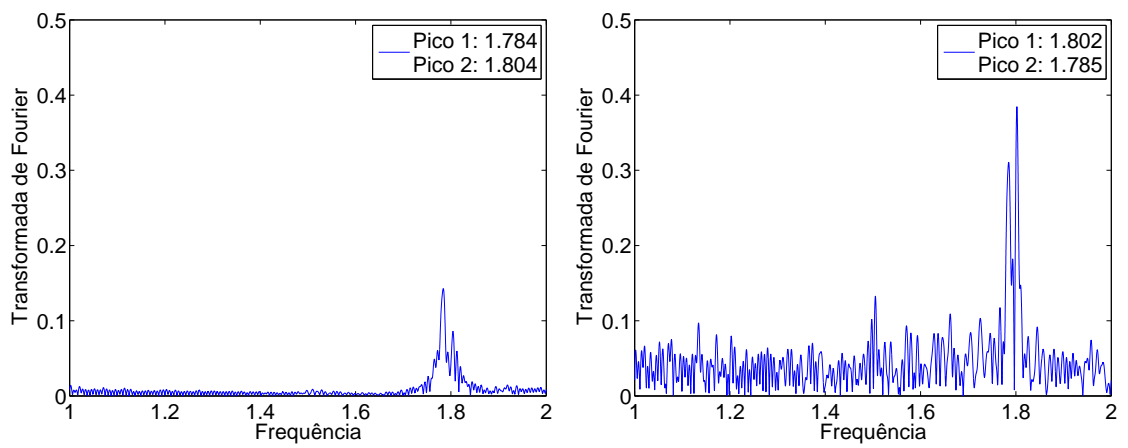

Fonte: Elaborada pelo autor.

Assim como no caso sem sóliton, observamos dois picos, referentes aos modos dipolar e breathing em ambos os gráficos, o que mostra novamente o acoplamento entre os dois modos devido à barreira. O acréscimo do sóliton aqui apenas faz com que esses modos percam energia, evidenciada pela diminuição dos picos, para o sóliton e outras oscilações, representadas pelo ruído nos gráficos.

\subsection{Sistema harmonicamente aprisionado com rugosi- dade no potencial}

Nessa seção ao invés de utilizarmos uma única barreira no centro da armadilha, vamos adicionar ao potencial harmônico um potencial senoidal de amplitude bem pequena, de forma que a excitação imposta ao BEC tenha frequência muito superior à imposta apenas por uma perturbação centralizada, isto é, a variação de tempo entre passagens consecutivas do sistema por perturbações passa de metade do período no caso da barreira central para o equivalente a um período do potencial senoidal nesse caso, mas de modo que cada uma das perturbações tenha intensidade bem pequena. Para cada sistema, vamos tratar dois casos, um em que o espaçamento entre as barreiras é uniforme e um em que os espaçamentos reduzem com a distância ao centro da armadilha, ou seja, temos a seguinte expressão para o potencial:

$$
V(x)=\frac{1}{2} \omega^{2} x^{2}+0,05 \mu \operatorname{sen}(a x)
$$

Sendo que temos duas situações: 
- Rugosidade uniformemente distribuída: $a=10$

- Rugosidade menos densa no centro: $a=\frac{\sqrt{3}}{\sqrt{x_{C M, 0}^{2}-x^{2}}},-x_{C M, 0}<x<x_{C M, 0}$

Onde a segunda foi deduzida de forma que a frequência imposta ao BEC é aproximadamente constante e igual à frequência do modo breathing: $\omega_{\text {breating }}=\sqrt{3}$.

\subsubsection{Condensado puro}

Vamos começar com um condensado puro deslocado do centro da armadilha, como mostrado na figura 3.42 .

Figura 3.42 - Condição inicial de um BEC inicialmente deslocado do centro da armadilha com rugosidade uniformemente distribuída

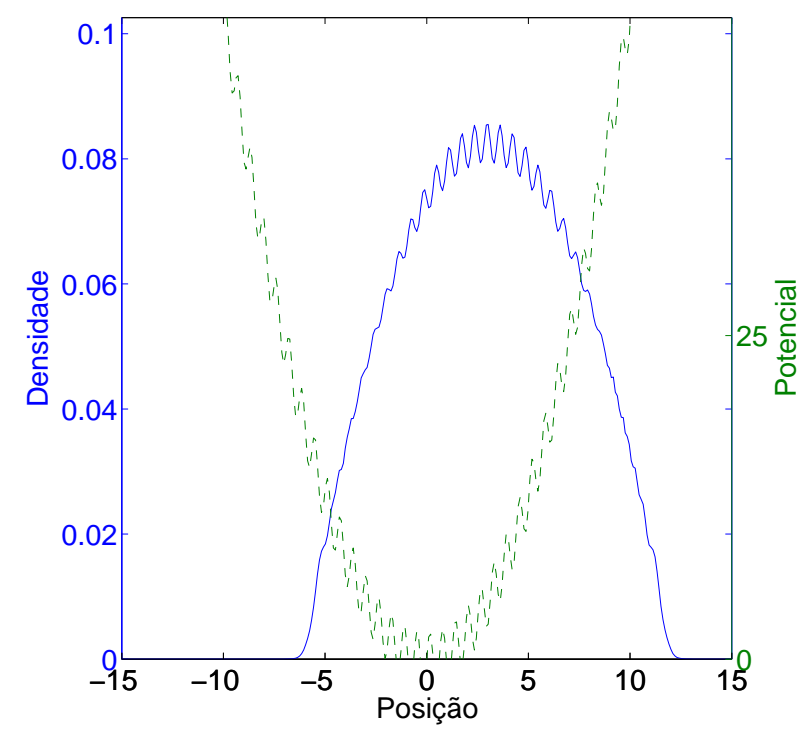

Fonte: Elaborada pelo autor.

Para os parâmetros utilizados nessa simulação, temos: $G_{0}=500, \omega=1$ e $x_{C M}=3,0$. Como em todos os casos anteriores, vamos observar inicialmente a imagem qualitativa (figura 3.43). 
Figura 3.43 - Imagem do movimento do BEC inicialmente deslocado do centro da armadilha com rugosidade uniformemente distribuída

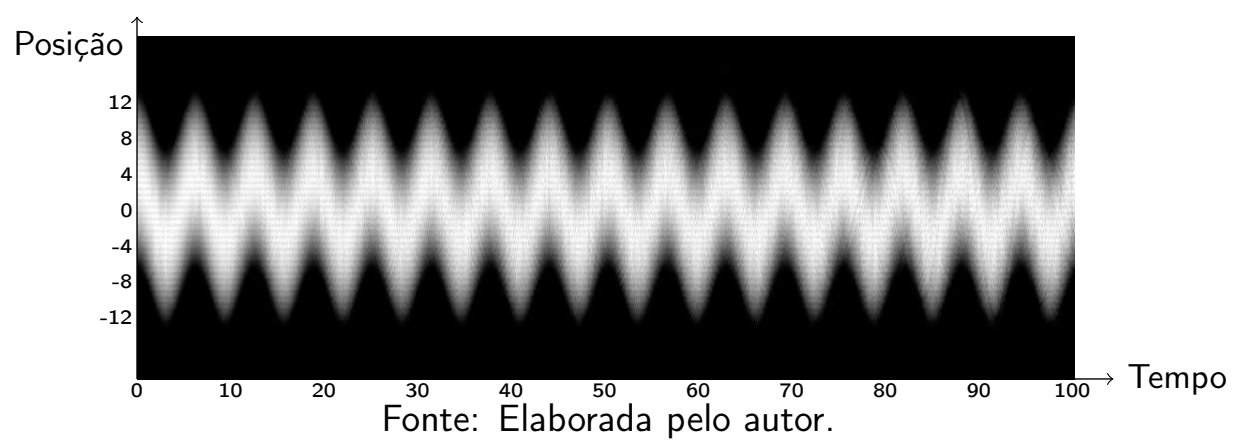

Nessa imagem há apenas leves ranhuras horizontais devido às ranhuras existentes no potencial. Para melhores análises, vamos observar o movimento do centro de massa desse sistema:

Figura 3.44 - Dinâmica do CM de um BEC inicialmente deslocado do centro de uma armadilha harmônica com rugosidade uniformemente distribuída
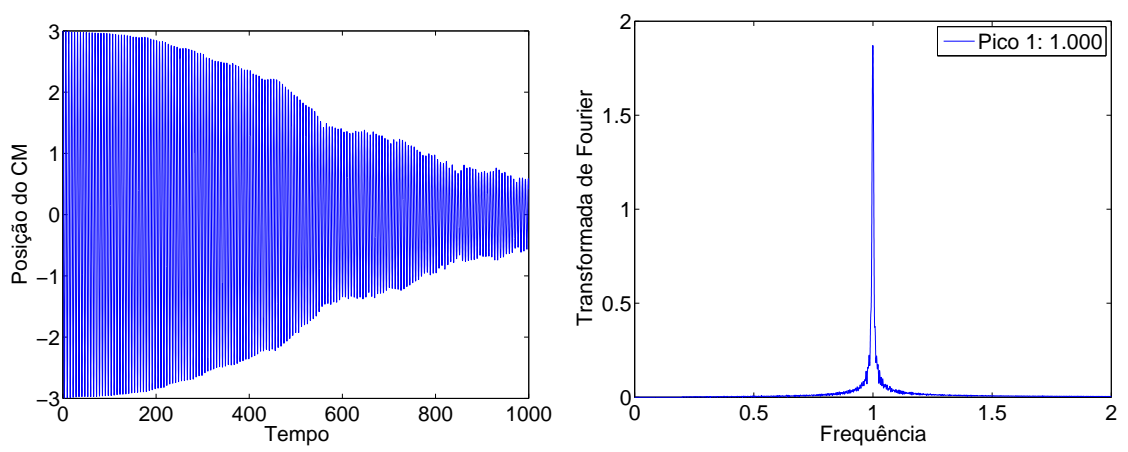

Fonte: Elaborada pelo autor.

Observe uma dinâmica exclusivamente do modo dipolar para o CM mas com uma enorme perda de energia em comparação com o caso de uma única barreira centralizada, em que não há perda de amplitude perceptível na escala de tempo utilizada.

Como o sistema é um sistema isolado, ou seja, como não consideramos dissipação nesse sistema, a energia dissipada nesse modo de oscilação tem que ser transferida para outro modo. Vamos observar a dinâmica do raio desse sistema na figura 3.49 . 
Figura 3.45 - Dinâmica do raio de um BEC inicialmente deslocado do centro de uma armadilha harmônica com rugosidade uniformemente distribuída
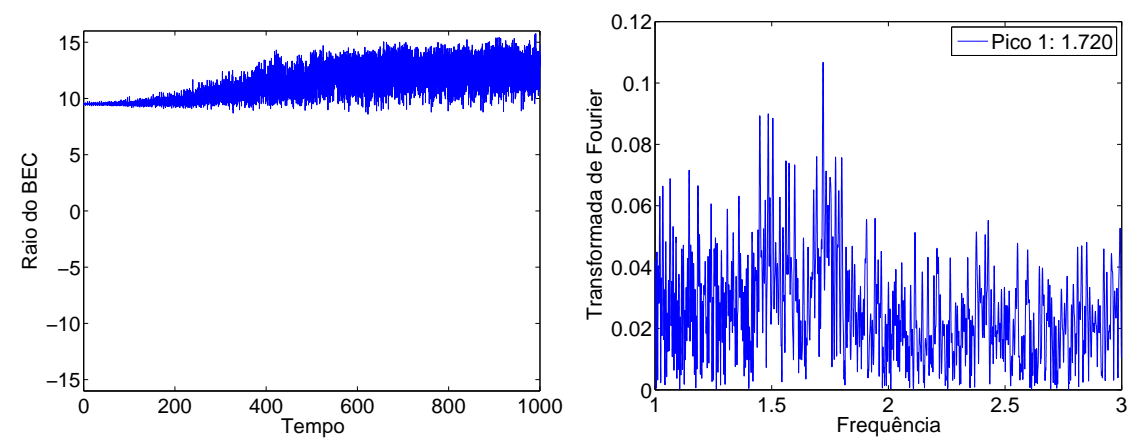

Fonte: Elaborada pelo autor.

Observe no primeiro gráfico que com a diminuição da amplitude de oscilação do CM há aumento da oscilação do raio, ou seja, há transferência de energia do modo dipolar para o modo breathing, porém, a partir da transformada de Fourier, pode-se observar que além do pico mais alto, com a frequência característica do modo breathing, há muitas outras frequências, isto porque a excitação do BEC nesse caso não é constante, afinal com apenas uma barreira centralizada o BEC demorava tempos iguais para passar pela barreira, já nesse caso, como as barreiras são uniformemente distribuídas, o intervalo de tempo para o BEC passar por duas barreiras consecutivas não é constante, fazendo com que o mesmo seja excitado por frequências diferentes dependentes da posição na armadilha.

Vamos agora ao potencial com a rugosidade menos densa no centro, com o objetico de facilitar a transferência de energia para o modo breathing (figura ??. 
Figura 3.46 - Condição inicial de um BEC inicialmente deslocado do centro da armadilha com rugosidade espcialmente não uniforme

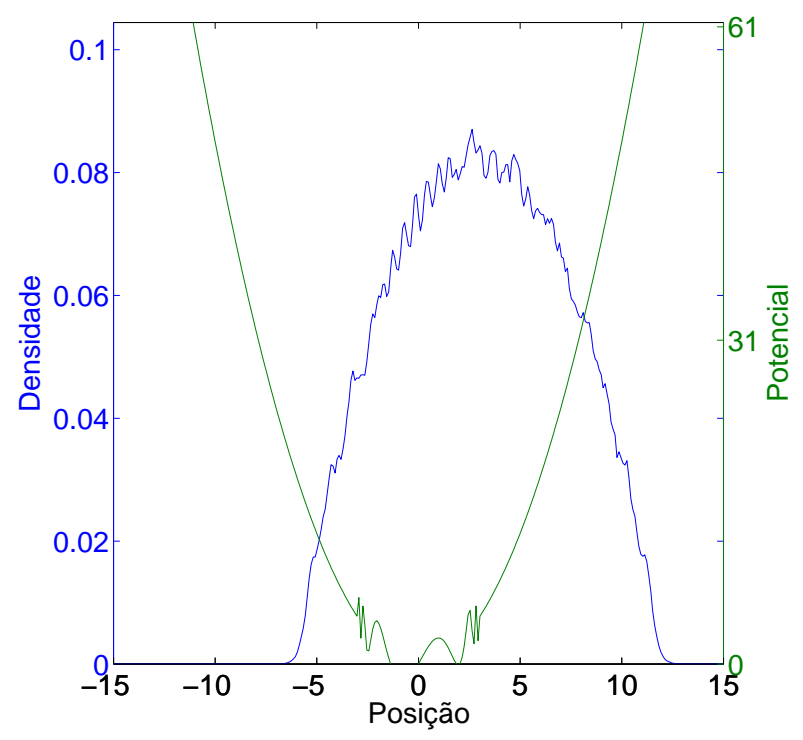

Fonte: Elaborada pelo autor.

Para essa simulação, utilizamos os seguintes parâmetros: $G_{0}=500, \omega=1$ e $x_{C M}=3,0$. Vamos observar os resultados iniciando com a imagem qualitativa (figura 3.47).

Figura 3.47 - Imagem do movimento do BEC inicialmente deslocado do centro da armadilha com rugosidade espcialmente não uniforme

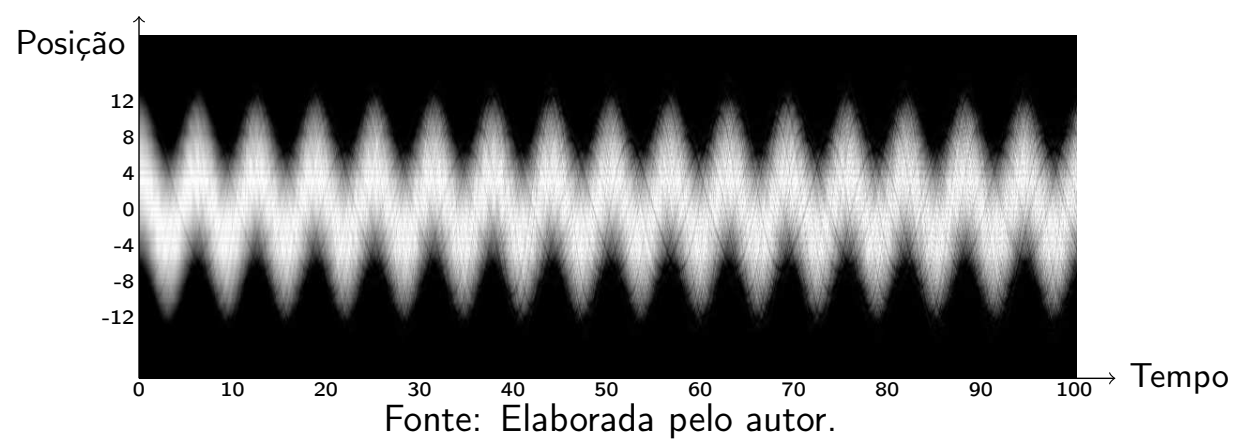

Não temos muito o que observar aqui. Novamente como no caso com rugosidade uniforme temos ranhuras horizontais devido às "pequenas barreiras", que agora não estão mais uniformemente distribuídas. Vamos ver no que isso afeta, começando pela dinâmica do CM (figura 3.59). 
Figura 3.48 - Dinâmica do CM de um BEC inicialmente deslocado do centro de uma armadilha harmônica com rugosidade espacialmente não uniforme
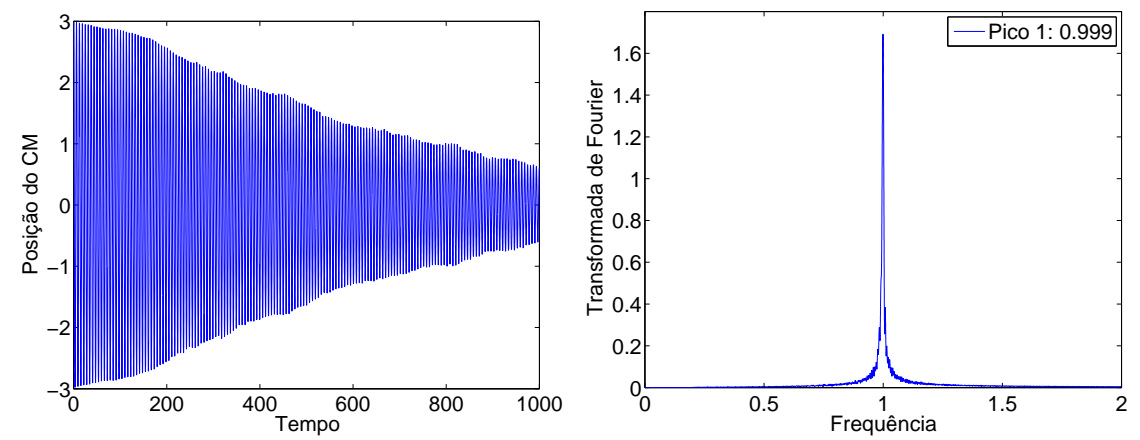

Fonte: Elaborada pelo autor.

Observe uma taxa de perda de energia do modo dipolar mais próxima da constância, isso porque agora a frequência de excitação é constante, o que faz com que menos modos sejam excitados. Vamos verificar o gráfico da dinâmica do raio na figura 3.49 para comprovar.

Figura 3.49 - Dinâmica do raio de um BEC inicialmente deslocado do centro de uma armadilha harmônica com rugosidade espacialmente não uniforme
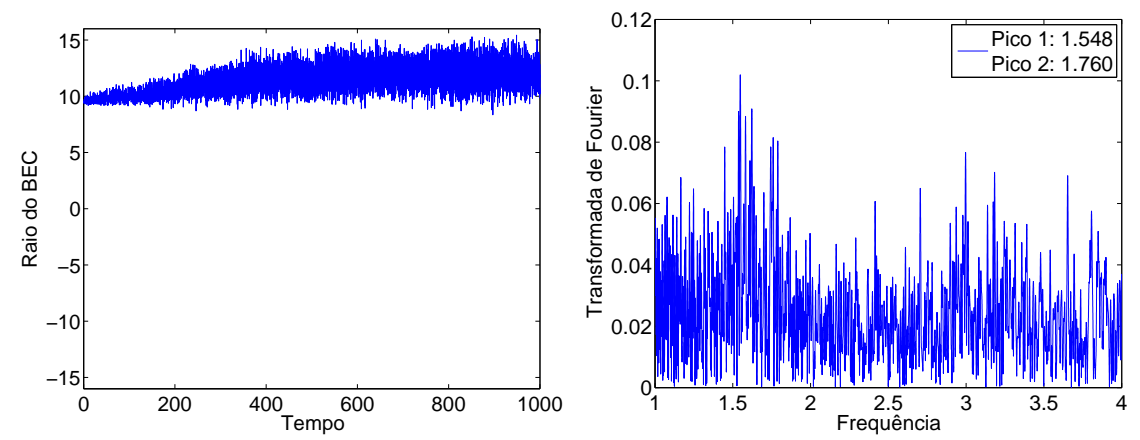

Fonte: Elaborada pelo autor.

Observe que apesar de ainda haver muitas frequências excitadas com as barreiras, obtivemos picos maiores na região da frequência relativa ao modo breathing, já que excitamos o sistema justamente com essa frequência.

\subsubsection{Condensado + Sóliton}

Agora adicionaremos sólitons ao sistema estudado. Para cada caso vamos comparar o sistema sem sóliton com os sistemas com cada um dos tipos de sóliton. Para as simulações, 
utilizamos os seguintes parâmetros: $G_{0}=500, \omega=1, x_{C M}=3,0, x_{0}=0$ e $u \in\{0 ; 0,5\}$. Como em todos os casos anteriores, vamos observar as condições iniciais 3.50 e as imagens qualitativas para os dois sólitons presentes no BEC (figura 3.51).

Figura 3.50 - Condições iniciais do sistema BEC+sóliton inicialmente deslocado do centro da armadilha com rugosidade uniformemente distribuída, para $u=0$ e $u=0,5$, respectivamente
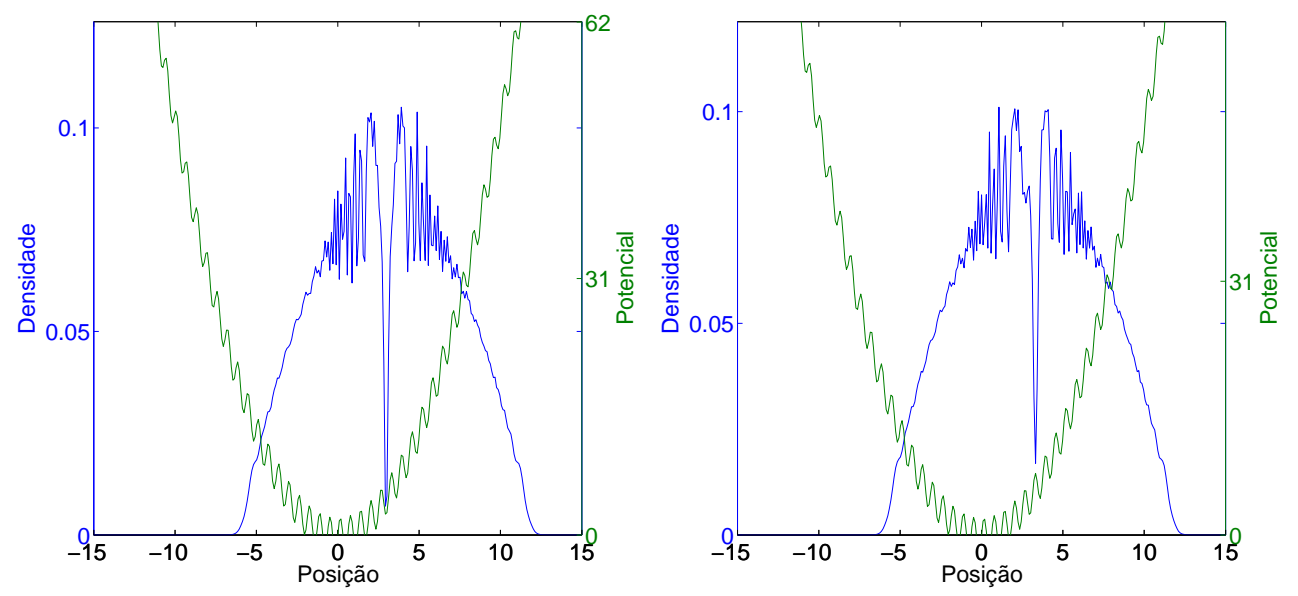

Fonte: Elaborada pelo autor.

Figura 3.51 - Imagens dos movimentos do BEC inicialmente deslocado do centro da armadilha com rugosidade uniformemente distribuída, para $u=0$ e $u=0,5$, respectivamente

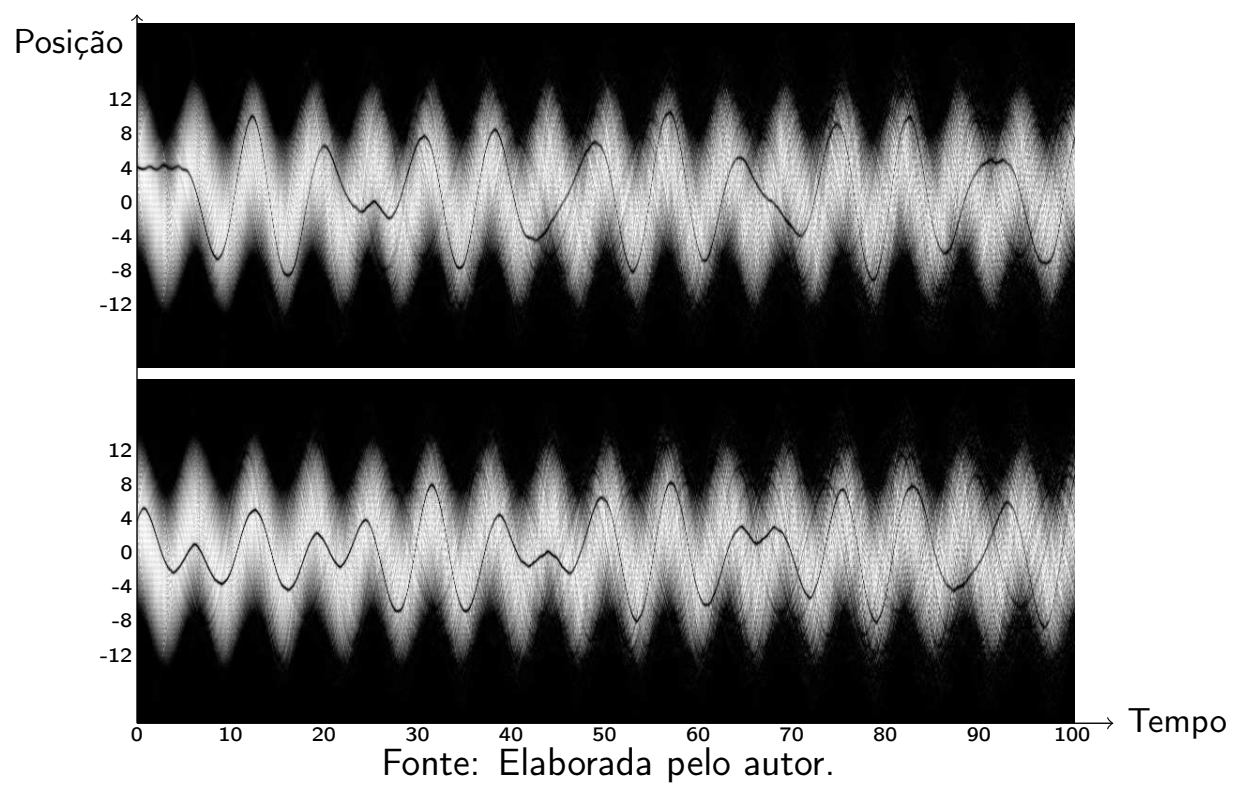

Observe que o black soliton fica inicialmente aprisionado entre duas barreiras até perder energia o suficiente para escapar e a partir daí se comporta como um grey soliton. Vamos analisar a dinâmica do CM nesses dois casos (figura [3.59). 
Figura 3.52 - Dinâmica do CM de um BEC inicialmente deslocado do centro de uma armadilha harmônica com rugosidade uniformemente distribuída, para $u=0$ e $u=0,5$, respectivamente
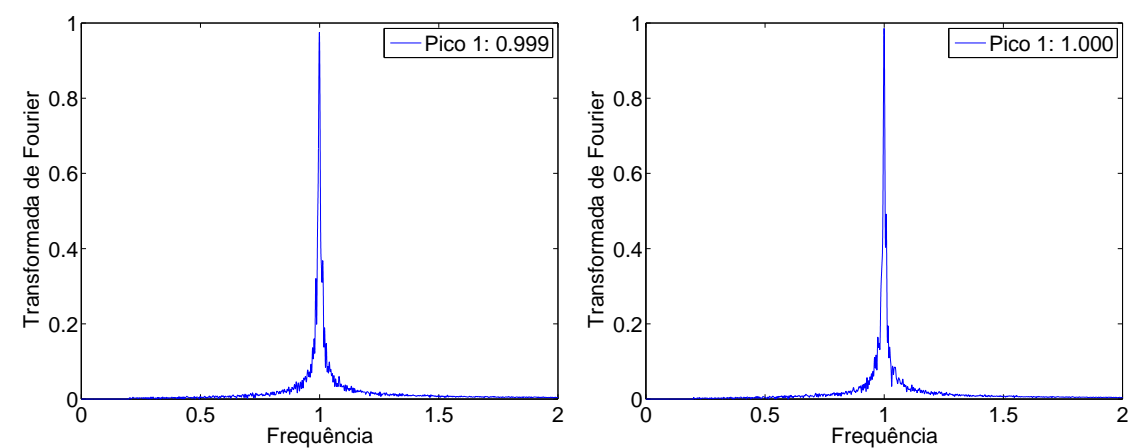

Fonte: Elaborada pelo autor.

Ambos os sistemas, apesar de ligeiramente diferentes não alteram o comportamento do centro de massa do sistema, afinal sua energia inicial é bem superior à energia das ondas sonoras emitidas pelo sóliton. Vamos à dinâmica do sóliton (figura 3.58).

Figura 3.53 - Dinâmica do sóliton em um BEC inicialmente deslocado do centro de uma armadiIha harmônica com rugosidade uniformemente distribuída, para $u=0$ e $u=0,5$, respectivamente
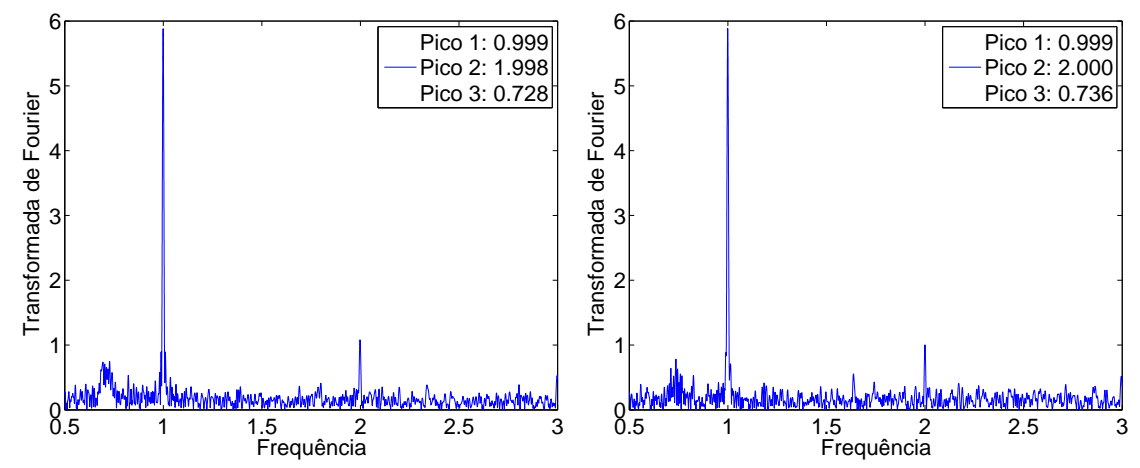

Fonte: Elaborada pelo autor.

Ao observarmos a dinâmica do sóliton, obtemos três picos de frequência, relativos ao próprio sóliton, ao modo dipolar e a uma segunda excitação do modo dipolar com energia superior, devido às várias frequências sentidas pelo sóliton em seu caminho. Vamos analisar a dinâmica do raio do BEC para vermos as consequências da presença do sóliton (figura 3.59). 
Figura 3.54 - Dinâmica do raio de um BEC inicialmente deslocado do centro de uma armadilha harmônica com rugosidade uniformemente distribuída, para $u=0$ e $u=0,5$, respectivamente
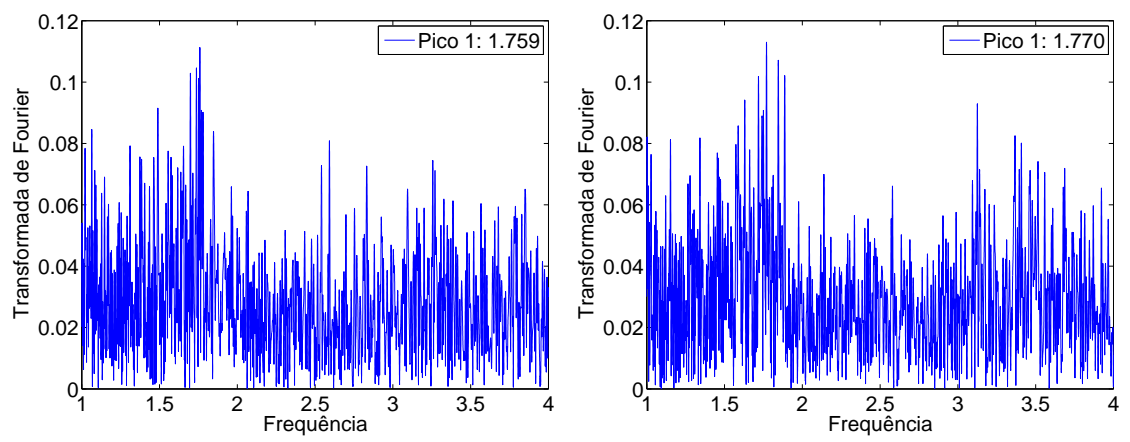

Fonte: Elaborada pelo autor.

Observe que o sóliton concentra as frequências excitadas mais próximo da frequência do modo breathing, ou seja, o sóliton facilita a transferência de energia do modo dipolar para esse modo em relação à transferência para outros modos.

Novamente usando o potencial com espaçamento não uniforme e utilizando os seguintes parâmetros: $G_{0}=500, \omega=1, x_{C M}=3,0, x_{0}=0$ e $u \in\{0 ; 0,5\}$. Como em todos os casos anteriores, vamos observar na figura 3.55 as condições iniciais do nosso sistema e na 3.56 as imagens qualitativas para os dois sólitons presentes no BEC.

Figura 3.55 - Condições iniciais do sistema BEC+sóliton inicialmente deslocado do centro da armadilha com rugosidade espacialmente não uniforme, para $u=0$ e $u=0,5$, respectivamente
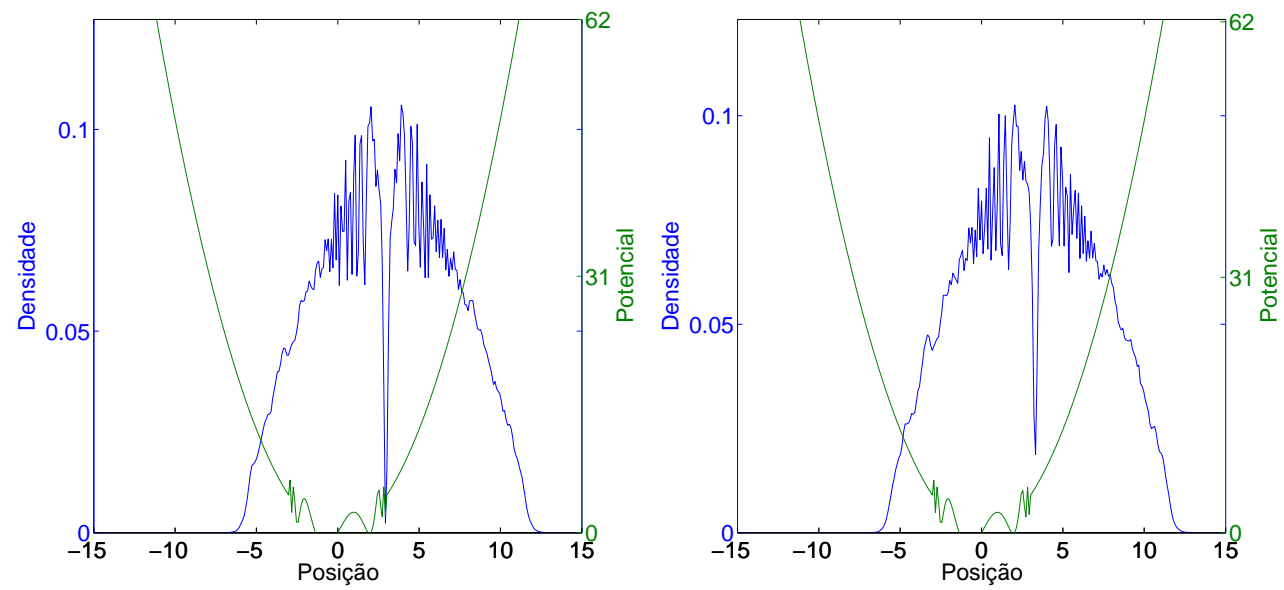

Fonte: Elaborada pelo autor. 
Figura 3.56 - Imagens dos movimentos do sistema BEC+sóliton inicialmente deslocado do centro na armadilha com rugosidade espacialmente não uniforme, para $u=0$ e $u=0,5$, respectivamente

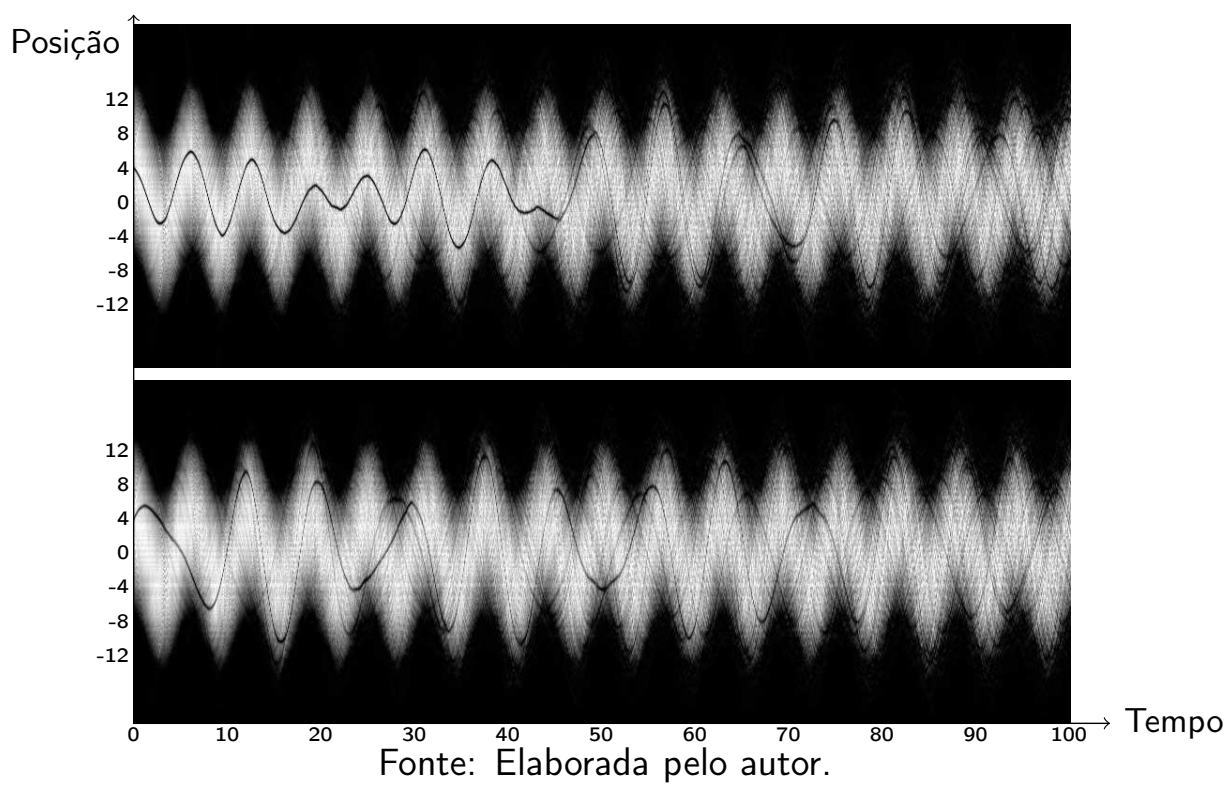

Observe a perda rápida de energia, caracterizada pela diminuição da depressão do sóliton. Vamos analisar na figura 3.57 a dinâmica do CM nesses dois casos.

Figura 3.57 - Dinâmica do CM de um sistema BEC+sóliton inicialmente deslocado do centro de uma armadilha harmônica com rugosidade espacialmente não uniforme, para $u=0 \mathrm{e}$ $u=0,5$, respectivamente
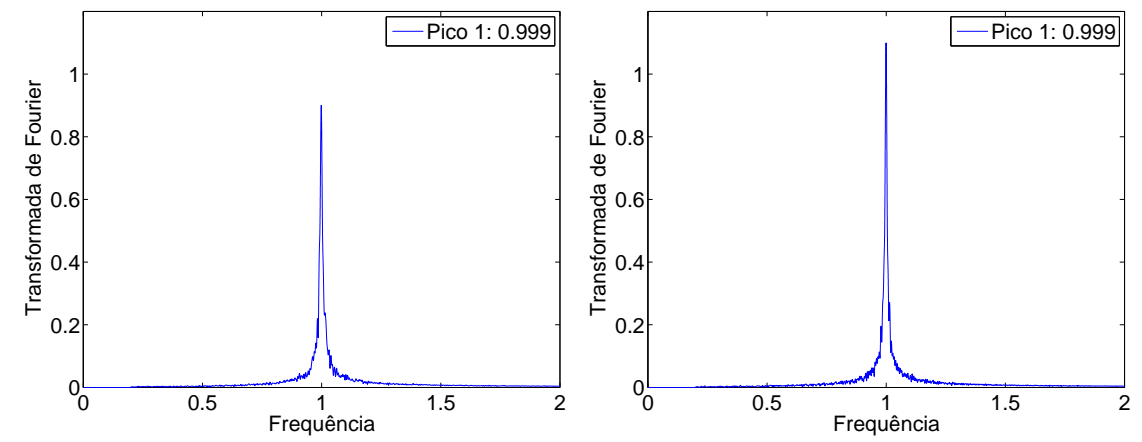

Fonte: Elaborada pelo autor.

Novamente a presença do sóliton não altera significativamente o movimento do BEC, já que a energia do modo dipolar é bem superior à das ondas sonoras emitidas pelo sóliton. Vamos à dinâmica do sóliton na figura 3.58 . 
Figura 3.58 - Dinâmica do sóliton em um BEC inicialmente deslocado do centro de uma armadilha harmônica com rugosidade espacialmente não uniforme, para $u=0$ e $u=0,5$, respectivamente
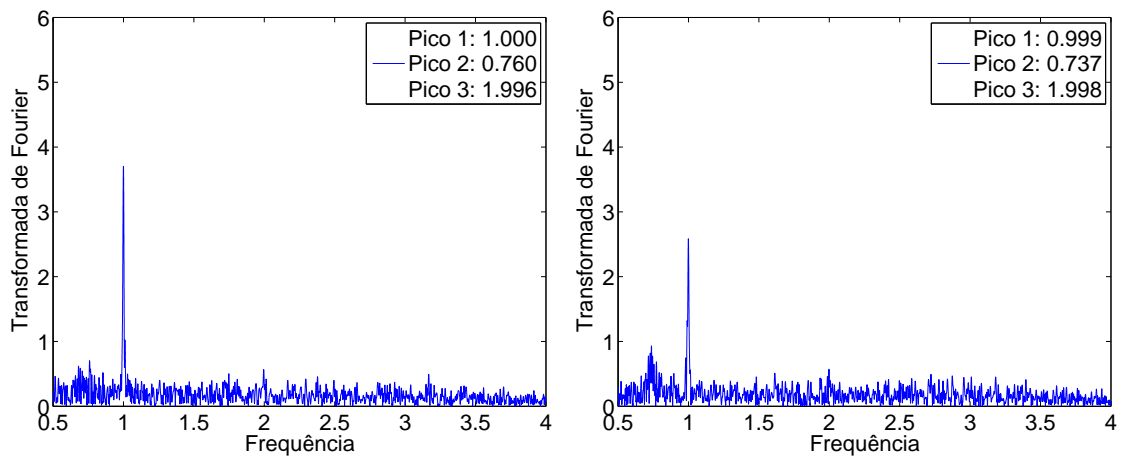

Fonte: Elaborada pelo autor.

Observamos aqui os mesmos picos que no caso do espaçamento constante, o que difere essa dinâmica da anterior é a menor amplitude de oscilação dos dois modos, o que indica uma perda de energia para outros modos. Vamos analisar a dinâmica do raio para vermos as consequências da presença do sóliton (figura 3.59).

Figura 3.59 - Dinâmica do raio de um BEC inicialmente deslocado do centro de uma armadilha harmônica com rugosidade espacialmente não uniforme, para $u=0$ e $u=0,5$, respectivamente
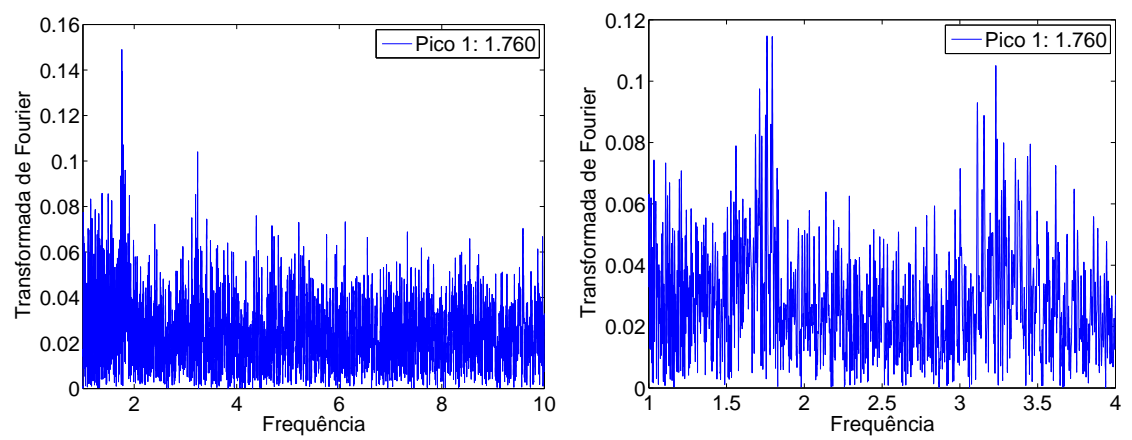

Fonte: Elaborada pelo autor.

Observe que novamente a presença dos sólitons aqui, apesar de gerar mais ruídos, aumenta o pico relativo ao modo breathing e concentra ainda mais as frequências em na região desse modo, já que excitamos justamente esse modo através da armadilha e o sóliton já o excita naturalmente. 


\section{Comparação com a Teoria de múltiplos orbitais}

A proposta inicial do nosso projeto era averiguar o tunelamento de um sistema composto, isto é, o BEC com vórtice, em analogia ao tunelamento de um sistema formado por duas entidades interagentes. Como exemplo temos uma molécula diatômica, com respectiva energia de ligação entre os seus átomos. Resolvemos estudar um sistema alternativo em 1D: um BEC com um dark-soliton, estrutura essa que pode inclusive gerar pares ou anéis de vórtices em seu decaimento, quando gerada respectivamente em sistema 2D e 3D, mas que também apresenta uma série características próprias.

Há muitos estudos tratando o tunelamento de um condensado no estado fundamental ou a interação de um sóliton com barreira potencial. Baseados nessas análises, caracterizamos a dinâmica de tunelamento de um BEC aprisionado em armadilha harmônica provido de um dark-soliton. Nos experimentos numéricos utilizamos as equações de campo médio, com a impressão do soliton no centro de massa do BEC, inicialmente deslocado do mínimo do potencial harmônico, local onde foi adicionada a nossa barreira potencial.

Alguns dos nossos resultados puderam ser explicados por meio de predições analítica da chamada aproximação de Thomas-Fermi. Ao final, comparamos as simulações de campo médio (equação de Gross-Pitaevskii) com as advindas da teoria de múltiplos orbitais a fim de justificar o regime de validade da nossa teoria.

Não só pela facilidade em aplicar métodos computacionais e análises analíticas, a razão de termos escolhido esse sistema é que ele pode vir a apresentar transição entre os regimes quântico e corpuscular, dependendo da razão entre suas energia cinética e de interação. Vale lembrar que o tunelamento dos bright solitons (estado estável de um condensado com interação atômica atrativa, que se propaga no espaço livre com preservação do seu perfil de densidade inicial) já foi bastante explorado em trabalhos da área, com a caracterização da sua dualidade onda-partícula através da medida da taxa de transmissão em barreira potencial. Em nosso projeto, porém, consideramos como nosso "pacote de onda" o condensado com interação atômica repulsiva provido de um dark-soliton, armadilhados em potencial harmônico. O modo 
dipolar do BEC nos fornecerá a dinâmica necessária para analisarmos sua travessia por barreira potencial, convenientemente acrescida no centro (mínimo) da armadilha.

Dentre as perspectivas para o projeto temos a possibilidade de trilhar o regime de validade das equações que evoluem o nosso sistema, as quais se baseiam na teoria de campo médio. As propriedades do dark-soliton, como sua dinâmica característica em sistemas inomogêneos (isto é, BEC submetido a um potencial externo, que se traduz em um potencial efetivo para o sóliton), tempo de vida e mesmo suas transmissão/reflexão por barreira são extremamente afetadas dependendo do regime do background. Na dinâmica de um sóliton em condensado na armadilha harmônica, por exemplo, existe um modo de oscilação não amortecida do sóliton devido a um delicado equilíbrio de energia, onde essa estrutura irradia e absorve energia através de ondas sonoras. Esse processo é bastante instável perante flutuações quânticas e térmicas, as quais chegariam a invalidar também um tratamento do sistema com a GPE, cuja derivação se baseia na função de onda do sistema dada por estados de particula única não correlacionados. Para isso visamos simular diferentes situações envolvendo a dinâmica dos sólitons e comparar os nosso resultados da GPE com aqueles advindos de teorias generalizadas, tal qual a teoria dos múltiplos orbitais (MCTDHB). Para cercar os limites do tratamento com campo médio, verificaremos também possibilidade de simular com MCTDHB a reflexão/transmissão parcial da nossa onda de sóliton através de barreira potencial, isto é, a possibilidade de fragmentar o estado inicial com a ocupação quântica macroscópica de dois outros estados correlacionados.

Aqui, vamos estudar o sistema mais simples contendo um sóliton, apenas para validar essa teoria que leva em consideração os vários estados de energia do sistema, comparando o tempo de desintegração do sóliton entre as duas teorias, além de verificar a fragmentação do sistema para cada caso utilizando um sistema de 2 orbitais. Para a simulação utilizamos um programa disponibilizado pelo criador da teoria.(10) Foram feitas para esse sistema simulações unidimensionais com 1 e 2 orbitais. Perceba que o sistema composto por apenas 1 orbital nada mais é que a própria GPE, ou seja, a GPE é um caso específico da teoria de múltiplos orbitais, portanto esse caso pode ser considerado para uma transição entre as duas teorias.

Aqui trataremos de um BEC centrado na armadilha contendo um grey soliton. Vamos analisar a evolução temporal da função de onda para um período de dinâmica que a presença do sóliton, através da perturbação causada pelas ondas sonoras, causa em um sistema de dois níveis.

Primeiramente vamos observar a evolução temporal do sistema já estudado da GPE, usando a simulação em xmds2 (12) (figura 4.1). 
Figura 4.1 - Evolução da função de onda de um BEC+sóliton inicialmente centrado na armadilha (simulação da GPE) para $t \in\left\{0 ; \frac{T}{4} ; \frac{T}{2} ; \frac{3 T}{4} ; T\right\}$, respectivamente, sendo $T$ o período de oscilação do sóliton
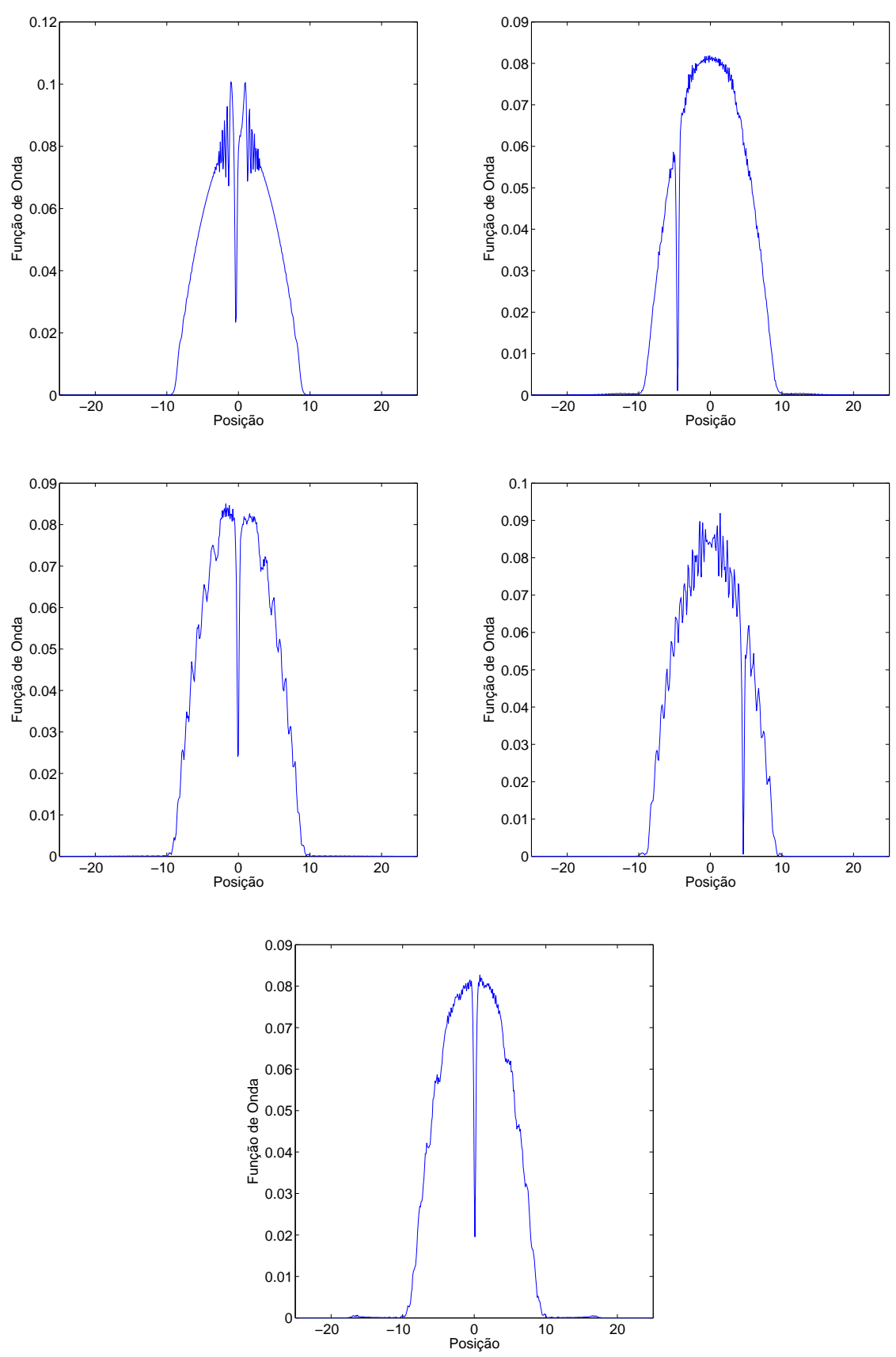

Fonte: Elaborada pelo autor.

Observe que não há perda visível de energia conforme a GPE e o mesmo observamos para a teoria de múltiplos orbitais, mas utilizando apenas um orbital, já que a GPE descreve exatamente um sistema de apenas um estado de energia, obtemos a evolução temporal mostrada na figura 4.2 . 
Figura 4.2 - Evolução da função de onda de um BEC+sóliton inicialmente centrado na armadilha (simulação MCTDHB com $M=1$ ) para $t \in\left\{0 ; \frac{T}{4} ; \frac{T}{2} ; \frac{3 T}{4} ; T\right\}$, respectivamente, sendo $T$ o período de oscilação do sóliton
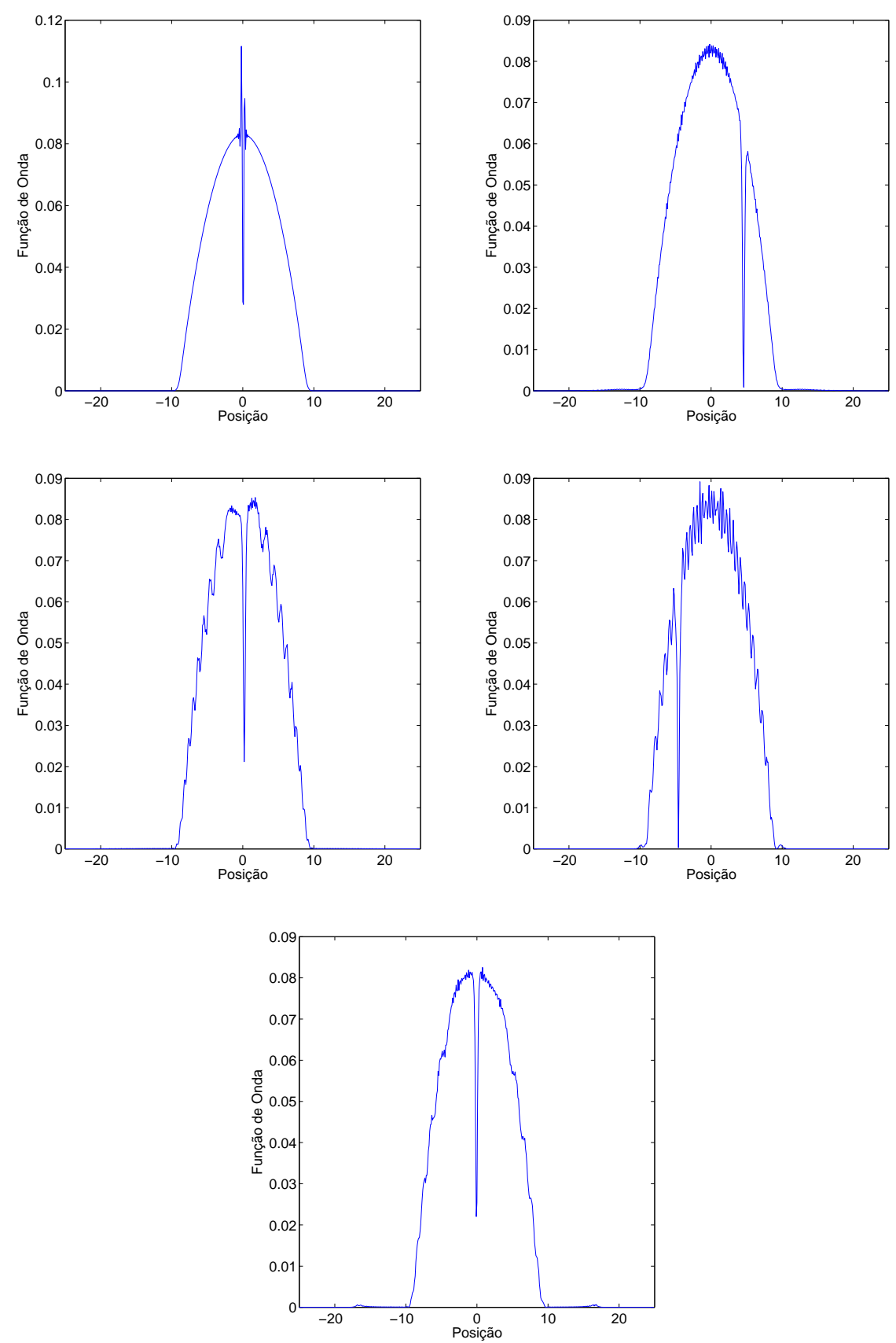

Fonte: Elaborada pelo autor.

Mas, conforme um dos criadores da teoria, Axel U. J. Lode, devido à fraca correlação da nossa condição inicial entre os orbitais, temos um rápido decaimento do sóliton, o que nem nos permite estudar esse sistema, como mostrado na figura 4.3 . 
Figura 4.3 - Evolução da função de onda de um BEC+sóliton inicialmente centrado na armadilha (simulação MCTDHB com $M=2$ ) para $t \in\left\{0 ; \frac{T}{8} ; \frac{T}{4}\right\}$, respectivamente, sendo $T \circ$ período de oscilação do sóliton
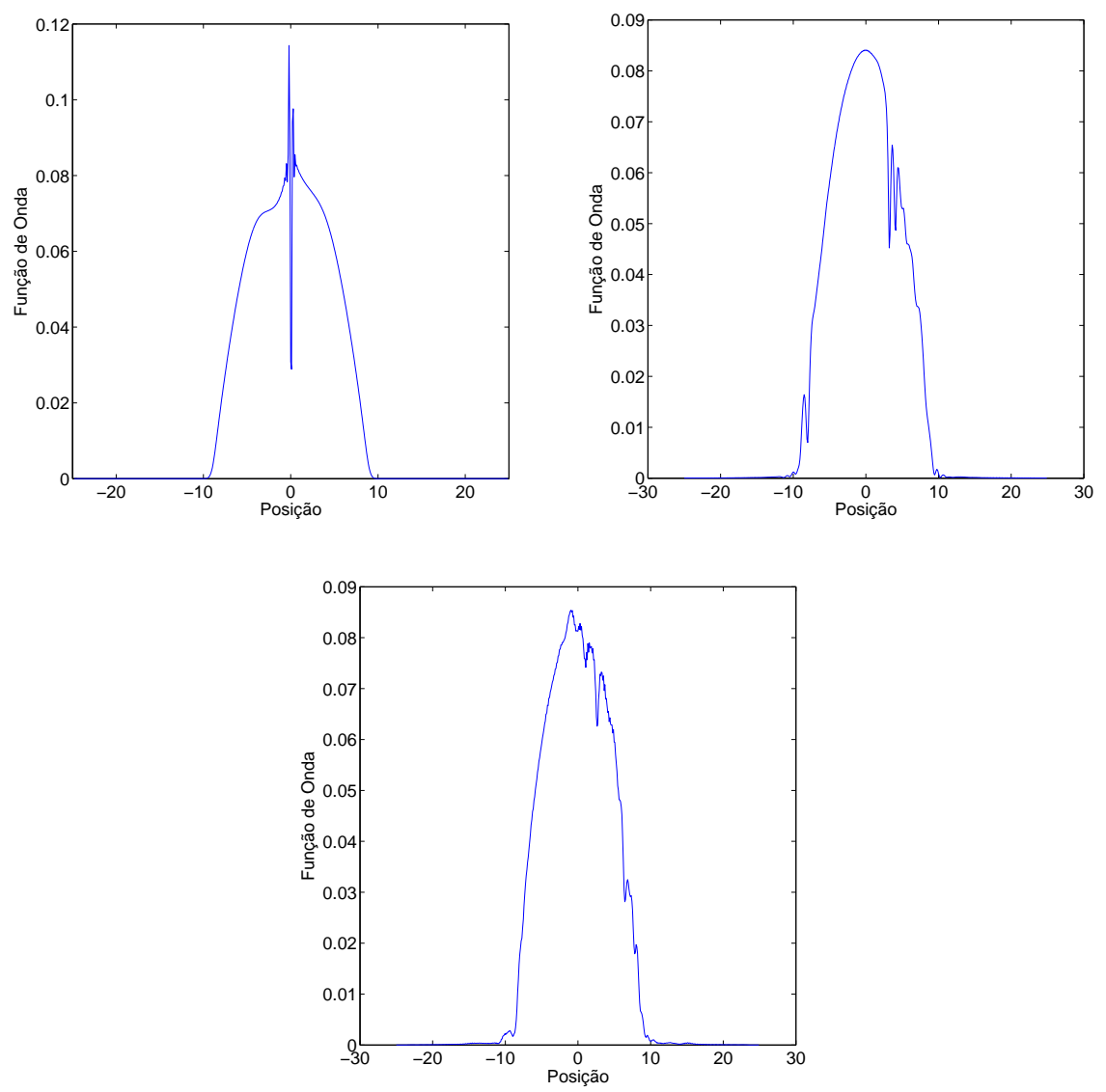

Fonte: Elaborada pelo autor. 


\section{Conclusão}

Nesse trabalho simulamos o sistema composto condensado+sóliton em três situações: na ausência e na presença de barreira e com uma rugosidade ao longo de todo o potencial.

No sistema sem barreira, observamos uma dinâmica composta pelo movimento do centro de massa em relação à armadilha e pelo movimento do sóliton em relação ao centro de massa, com trocas de energia entre o modo dipolar do BEC e o sóliton através de ondas sonoras emitidas e reabsorvidas pelo sóliton.

A presença da barreira nos trouxe alguns fenômenos, seja eles relacionados à presença dos sólitons ou não. Em relação ao BEC temos a geração de sólitons em um BEC oscilante com barreira e o acoplamento dos modos dipolar e breathing. Em relação aos sólitons, temos o comportamento anti-damping do sóliton, as divisões dos sólitons através do comportamento ondulatório do sóliton, representado pela reflexão/transmissão do mesmo nas barreiras mais baixas e o comportamento corpuscular do sóliton, representado pela total reflexão nas barreiras mais altas.

Em seguida utilizamos um potencial uniformemente rugoso que fez com que houvesse uma rápida perda de energia do modo dipolar para outros modos, gerando muito ruído no espectro devido às ondas sonoras geradas e à excitação em um espectro contínuo de frequências, mas destacando-se o modo breathing. Alterando esse potencial para que houvesse uma excitação de frequências mais concentradas na região de frequências do modo breathing, obtivemos um espectro com picos mais concentrados nessa região. A inclusão do sóliton nesse sistema favoreceu essa transmissão, concentrando ainda mais os picos de frequência na região da frequência do modo breathing.

Por último comparamos a teoria de campo médio de Gross-Pitaevskii com a teoria de múltiplos orbitais (MCTDHB) para um sistema contendo um sóliton. Utilizando a última com apenas um orbital, obtemos o mesmo comportamento da teoria de campo médio. Já considerando dois orbitais, o tempo de vida do sóliton foi extremamente curto (menos de um quarto do período de oscilação do sóliton), contrariando resultados experimentais, isso ocorre, conforme um criador da teoria, Axel U. J. Lode, devido à fraca correlação da nossa 
condição inicial entre os orbitais, pois a função que utilizamos para o sistema BEC+sóliton é uma aproximação, que não descreve exatamente a realidade observada nos experimentos.

Sumarizando nossos principais resultados:

- Validação de nossa simulação com resultados conhecidos

- Troca de energia entre o sóliton e o BEC causada por pequena barreira

- Geração de sólitons em condensado oscilante, com energia proveniente dos modos coletivos do BEC

- Acoplamento entre os modos dipolar e o breathing do BEC na presença de uma barreira com energia maior que a do BEC

- Transferência mais rápida de energia entre modos utilizando uma perturbação distribuída

- Picos de frequência mais definidos ao utilizarmos uma perturbação distribuída com frequência de excitação constante

- Transferência de energia para o modo breathing mais eficiente na presençã de sólitons

- Na teoria de múltiplos orbitais nosso sistema se mostrou pouco correlacionado, fazendo com que o sóliton decaísse muito rapidamente, ao contrário do que mostram os experimentos 


\section{REFERÊNCIAS}

1 FETTER, A. L.; SVIDZINSKY, A. A. Vortices in a trapped dilute Bose-Einstein condensate. Journal of Physics: condensed matter, v. 13, n. 12, p. R135, 2001.

2 PINDZOLA, M.; SUN, B. Collective modes of ground and excited states of Bose-Einstein condensates in axially symmetric anisotropic traps. Journal of Physics B: atomic, molecular and optical physics, v. 43, n. 23, p. 235302, 2010.

3 MENOTTI, C.; STRINGARI, S. Collective oscillations of a one-dimensional trapped BoseEinstein gas. Physical Review A, v. 66, n. 4, p. 043610, 2002.

4 PETHICK, C. J.; SMITH, H. Bose-Einstein condensation in dilute gases. 2nd ed. Cambridge: Cambridge University Press, 2008.

5 KETTERLE, W. Nobel lecture: when atoms behave as waves: Bose-Einstein condensation and the atom laser. Reviews of Modern Physics, v. 74, p. 1131-1151, 2002. doi: 10.1103/RevModPhys.74.1131.

6 SALINAS, S. R. A. Introduction to statistical physics. New York: Springer, 2001.

7 DALFOVO, F.; PITAEVSKII, L.; STRINGARI, S. The condensate wave function of a trapped atomic gas. Journal of Research of the National Institute of Standards and Technology, Washington, v. 101, n. 4, p. 537-544, 1996.

8 PARKER, N. G.; PROUKAKIS, N. P.; ADAMS, C. S. Dark soliton dynamics in confined Bose-Einstein condensates. In: PARKER, N. G.; PROUKAKIS, N. P.; ADAMS, C. S. Progress in soliton research. New York: Nova Publishing, 2005. p. 1-49.

9 BUSCH, T.; ANGLIN, J. Mossbauer effect for dark solitons in Bose-Einstein condensates. 1998. Disponível em: <http://arxiv.org/pdf/cond-mat/9809408v1.pdf>. Acesso em: 13 nov. 2014.

10 ALON, O.; STRELTSOV, A.; CEDERBAUM, L. Multiconfigurational time-dependent Hartree method for bosons: many-body dynamics of bosonic systems. Physics Review A, v. 77, p. 033613, 2008. doi: 10.1103/PhysRevA.77.033613.

11 MURUGANANDAM, P.; ADHIKARI, S. K. Fortran programs for the time-dependent Gross-Pitaevskii equation in a fully anisotropic trap. Computer Physics Communications, v. 180, n. 10, p. 1888-1912, 2009. 
12 DENNIS, G. R.; HOPE, J. J.; JOHNSSON, M. T. XMDS2: fast, scalable simulation of coupled stochastic partial differential equations. Computer Physics Communications, v. 184, n. 1, p. 201-208, 2013.

13 THEOCHARIS, G.; KEVREKIDIS, P.; NISTAZAKIS, H.; FRANTZESKAKIS, D.; BISHOP, A. Generation of dark solitons in oscillating Bose-Einstein condensates. Physics Letters A, v. 337, n. 4, p. 441-448, 2005.

14 SAKAGUCHI, H.; TAMURA, M. Scattering of solitons and dark solitons by potential walls in the nonlinear Schrödinger equation. Journal of the Physical Society of Japan, v. 74, n. 1, p. $292-298,2005$.

15 HUANG, G.; SZEFTEL, J.; ZHU, S. Dynamics of dark solitons in quasi-one-dimensional Bose-Einstein condensates. Physical Review A, v. 65, n. 5, p. 053605, 2002.

16 FRANTZESKAKIS, D. J.; THEOCHARIS, G.; DIAKONOS, F. K.; SCHMELCHER, P.; KIVSHAR, Y. S. Interaction of dark solitons with localized impurities in Bose-Einstein condensates. Physical Review A, v. 66, p. 053608, 2002. doi: 10.1103/PhysRevA.66.053608.

17 FRANTZESKAKIS, D. Dark solitons in atomic Bose-Einstein condensates: from theory to experiments. Journal of Physics A: mathematical and theoretical, v. 43, n. 21, p. 213001, 2010. 
APENNDICE $A$

\section{Constantes Físicas}

Tabela A.1 - Constantes Físicas

\begin{tabular}{r|c|c} 
Constante & Símbolo & Valor (SI) \\
\hline Constante de Boltzmann & $k_{B}$ & $1,3806503 \times 10^{-23} \mathrm{~J} / \mathrm{K}$ \\
Constante de Planck reduzida & $\hbar$ & $1,054571726(47) \times 10^{-34} \mathrm{~J} \cdot \mathrm{s}$ \\
Fonte: Elaborada pelo autor.
\end{tabular}

Fonte: Elaborada pelo autor 
APENNDICE $B$

\section{Dedução do modo dipolar}

Vamos deduzir esse resultado a partir do método variacional, utilizando a seguinte densidade de Lagrangiana:

$$
\mathcal{L}=\underbrace{\frac{i}{2} \hbar\left(\psi \frac{\partial \psi^{*}}{\partial t}-\psi^{*} \frac{\partial \psi}{\partial t}\right)}_{\mathcal{L}_{1}}+\underbrace{\frac{\hbar^{2}}{2 m}|\nabla \psi|^{2}}_{\mathcal{L}_{2}}+\underbrace{V(x)|\psi|^{2}}_{\mathcal{L}_{3}}+\underbrace{\frac{1}{2} U_{0}|\psi|^{4}}_{\mathcal{L}_{4}}
$$

e o seguinte ansatz para a função de onda:

$$
\psi(x, t)=A(t) \mathrm{e}^{-\frac{\left[x-x_{0}(t)\right]^{2}}{2 w(t)^{2}}+i x \alpha(t)+i x^{2} \beta(t)}
$$

Vamos calcular cada uma das partes da densidade de Lagrangiana:

$$
\begin{aligned}
\mathcal{L}_{1} & =\frac{i}{2} \hbar\left(\psi \frac{\partial \psi^{*}}{\partial t}-\psi^{*} \frac{\partial \psi}{\partial t}\right) \\
& =\frac{i}{2} \hbar\left\{A \mathrm{e}^{-\frac{\left(x-x_{0}\right)^{2}}{w^{2}}}\left[\dot{A}^{*}+A^{*}\left(\frac{\left(x-x_{0}\right) w^{2} \dot{x}_{0}+w \dot{w}\left(x-x_{0}\right)^{2}}{w^{4}}-i x \dot{\alpha}-i x^{2} \dot{\beta}\right)\right]-A^{*} \mathrm{e}^{-\frac{\left(x-x_{0}\right)^{2}}{w^{2}}}\left[\dot{A}+A\left(\frac{\left(x-x_{0}\right) w^{2} \dot{x}_{0}+w \dot{w}\left(x-x_{0}\right)^{2}}{w^{4}}+i x \dot{\alpha}+i x^{2} \dot{\beta}\right)\right]\right\} \\
& =\frac{i}{2} \hbar \mathrm{e}^{-\frac{\left(x-x_{0}\right)^{2}}{w^{2}}}\left[A \dot{A}^{*}-A^{*} \dot{A}-2 i|A|^{2} x(\dot{\alpha}+\dot{\beta} x)\right] \\
& =\frac{i}{2} \hbar\left(A \dot{A}^{*}-A^{*} \dot{A}\right) \mathrm{e}^{-\frac{\left(x-x_{0}\right)^{2}}{w^{2}}}+\hbar|A|^{2} \dot{\alpha} x \mathrm{e}^{-\frac{\left(x-x_{0}\right)^{2}}{w^{2}}}+\hbar|A|^{2} \dot{\beta} x^{2} \mathrm{e}^{-\frac{\left(x-x_{0}\right)^{2}}{w^{2}}}
\end{aligned}
$$




$$
\begin{aligned}
& \mathcal{L}_{2}=\frac{\hbar^{2}}{2 m}\left|\frac{\partial \psi}{\partial x}\right|^{2} \\
& =\frac{\hbar^{2}}{2 m}\left|\frac{\partial}{\partial x}\left[A \mathrm{e}^{-\frac{\left(x-x_{0}\right)^{2}}{2 w^{2}}+i x \alpha+i x^{2} \beta}\right]\right|^{2} \\
& =\frac{\hbar^{2}}{2 m}|A|^{2}\left|\left[-\frac{\left(x-x_{0}\right)}{w^{2}}+i(\alpha+2 x \beta)\right] \mathrm{e}^{-\frac{\left(x-x_{0}\right)^{2}}{2 w^{2}}+i x \alpha+i x^{2} \beta}\right|^{2} \\
& =\frac{\hbar^{2}}{2 m}|A|^{2} \mathrm{e}^{-\frac{\left(x-x_{0}\right)^{2}}{w^{2}}}\left[\frac{\left(x-x_{0}\right)^{2}}{w^{4}}+(\alpha+2 x \beta)^{2}\right] \\
& =\frac{\hbar^{2}}{2 m w^{4}}|A|^{2}\left(x-x_{0}\right)^{2} \mathrm{e}^{-\frac{\left(x-x_{0}\right)^{2}}{w^{2}}}+\frac{\hbar^{2}}{2 m}|A|^{2}(\alpha+2 x \beta)^{2} \mathrm{e}^{-\frac{\left(x-x_{0}\right)^{2}}{w^{2}}} \\
& \mathcal{L}_{3}=V(x)|\psi|^{2} \\
& =\left(\frac{1}{2} m \omega^{2} x^{2}\right)\left|A \mathrm{e}^{-\frac{\left(x-x_{0}\right)^{2}}{2 w^{2}}+i x \alpha+i x^{2} \beta}\right|^{2} \\
& =\frac{1}{2} m \omega^{2}|A|^{2} x^{2} \mathrm{e}^{-\frac{\left(x-x_{0}\right)^{2}}{w^{2}}} \\
& \mathcal{L}_{4}=\frac{1}{2} U_{0}|\psi|^{4} \\
& =\frac{1}{2} U_{0}\left|A \mathrm{e}^{-\frac{\left(x-x_{0}\right)^{2}}{2 w^{2}}+i x \alpha+i x^{2} \beta}\right|^{4} \\
& =\frac{1}{2} U_{0}|A|^{4} \mathrm{e}^{-\frac{2\left(x-x_{0}\right)^{2}}{w^{2}}}
\end{aligned}
$$

Vamos agora ao cálculo da Lagrangiana:

$$
L=\int_{-\infty}^{\infty} \mathcal{L} d x \Rightarrow L_{i}=\int_{-\infty}^{\infty} \mathcal{L}_{i} d x
$$




$$
\begin{aligned}
L_{1} & =\int_{-\infty}^{\infty} \frac{i}{2} \hbar\left(A \dot{A}^{*}-A^{*} \dot{A}\right) \mathrm{e}^{-\frac{\left(x-x_{0}\right)^{2}}{w^{2}}}+\hbar|A|^{2} \dot{\alpha} x \mathrm{e}^{-\frac{\left(x-x_{0}\right)^{2}}{w^{2}}}+\hbar|A|^{2} \dot{\beta} x^{2} \mathrm{e}^{-\frac{\left(x-x_{0}\right)^{2}}{w^{2}}} d x \\
& =\frac{i}{2} \hbar\left(A \dot{A}^{*}-A^{*} \dot{A}\right) \int_{-\infty}^{\infty} \mathrm{e}^{-\frac{\left(x-x_{0}\right)^{2}}{w^{2}}} d x+\hbar|A|^{2} \dot{\alpha} \int_{-\infty}^{\infty} x \mathrm{e}^{-\frac{\left(x-x_{0}\right)^{2}}{w^{2}}} d x+\hbar|A|^{2} \dot{\beta} \int_{-\infty}^{\infty} x^{2} \mathrm{e}^{-\frac{\left(x-x_{0}\right)^{2}}{w^{2}}} d x \\
& =\frac{i}{2} \hbar\left(A \dot{A}^{*}-A^{*} \dot{A}\right) w \sqrt{\pi}+\hbar|A|^{2} \dot{\alpha} x_{0} w \sqrt{\pi}+\hbar|A|^{2} \dot{\beta}\left(x_{0}^{2}+\frac{w^{2}}{2}\right) w \sqrt{\pi} \\
& =\frac{i}{2} \hbar\left(A \dot{A}^{*}-A^{*} \dot{A}\right) w \sqrt{\pi}+\hbar|A|^{2} w \sqrt{\pi}\left[\dot{\alpha} x_{0}+\dot{\beta}\left(x_{0}^{2}+\frac{w^{2}}{2}\right)\right]
\end{aligned}
$$

$$
\begin{aligned}
L_{2} & =\int_{-\infty}^{\infty} \frac{\hbar^{2}}{2 m w^{4}}|A|^{2}\left(x-x_{0}\right)^{2} \mathrm{e}^{-\frac{\left(x-x_{0}\right)^{2}}{w^{2}}}+\frac{\hbar^{2}}{2 m}|A|^{2}(\alpha+2 x \beta)^{2} \mathrm{e}^{-\frac{\left(x-x_{0}\right)^{2}}{w^{2}}} d x \\
& =\frac{\hbar^{2}}{2 m w^{4}}|A|^{2} \int_{-\infty}^{\infty}\left(x-x_{0}\right)^{2} \mathrm{e}^{-\frac{\left(x-x_{0}\right)^{2}}{w^{2}}} d x+\frac{\hbar^{2}}{2 m}|A|^{2} \int_{-\infty}^{\infty}(\alpha+2 x \beta)^{2} \mathrm{e}^{-\frac{\left(x-x_{0}\right)^{2}}{w^{2}}} d x \\
& =\frac{\hbar^{2}}{2 m w^{4}}|A|^{2} \frac{w^{2}}{2} w \sqrt{\pi}+\frac{\hbar^{2}}{2 m}|A|^{2}\left[\left(\alpha+2 x_{0} \beta\right)^{2} w \sqrt{\pi}+2 \beta^{2} w^{3} \sqrt{\pi}\right] \\
& =\frac{\hbar^{2}}{2 m}|A|^{2} w \sqrt{\pi}\left[\left(\alpha+2 x_{0} \beta\right)^{2}+2 \beta^{2} w^{2}+\frac{1}{2} w^{-2}\right]
\end{aligned}
$$

$$
\begin{aligned}
L_{3} & =\int_{-\infty}^{\infty} \frac{1}{2} m \omega^{2}|A|^{2} x^{2} \mathrm{e}^{-\frac{\left(x-x_{0}\right)^{2}}{w^{2}}} d x \\
& =\frac{1}{2} m \omega^{2}|A|^{2} \int_{-\infty}^{\infty} x^{2} \mathrm{e}^{-\frac{\left(x-x_{0}\right)^{2}}{w^{2}}} d x \\
& =\frac{1}{2} m \omega^{2}|A|^{2} w \sqrt{\pi}\left(x_{0}^{2}+\frac{w^{2}}{2}\right) \\
L_{4} & =\int_{-\infty}^{\infty} \frac{1}{2} U_{0}|A|^{4} \mathrm{e}^{-\frac{2\left(x-x_{0}\right)^{2}}{w^{2}}} d x \\
& =\frac{1}{2} U_{0}|A|^{4} \int_{-\infty}^{\infty} \mathrm{e}^{-\frac{2\left(x-x_{0}\right)^{2}}{w^{2}}} d x \\
& =\frac{\frac{1}{2} U_{0}|A|^{4}}{\sqrt{2}} w \sqrt{\pi}
\end{aligned}
$$

Finalmente vamos à Lagrangiana total: 


$$
\begin{aligned}
L & =L_{1}+L_{2}+L_{3}+L_{4} \\
& =\frac{i}{2} \hbar\left(A \dot{A}^{*}-A^{*} \dot{A}\right) w \sqrt{\pi}+\hbar|A|^{2} w \sqrt{\pi}\left[\dot{\alpha} x_{0}+\dot{\beta}\left(x_{0}^{2}+\frac{w^{2}}{2}\right)\right]+\frac{\hbar^{2}}{2 m}|A|^{2} w \sqrt{\pi}\left[\left(\alpha+2 x_{0} \beta\right)^{2}+2 \beta^{2} w^{2}+\frac{1}{2} w^{-2}\right]+\frac{1}{2} m \omega^{2}|A|^{2} w \sqrt{\pi}\left(x_{0}^{2}+\frac{w^{2}}{2}\right)+\frac{\frac{1}{2} U_{0}|A|^{4}}{\sqrt{2}} w \sqrt{\pi} \\
& =\frac{i}{2} \hbar\left(A \dot{A}^{*}-A^{*} \dot{A}\right) w \sqrt{\pi}+|A|^{2} w \sqrt{\pi}\left\{\hbar\left(\dot{\alpha} x_{0}+\dot{\beta} x_{0}^{2}+\dot{\beta} \frac{w^{2}}{2}\right)+\frac{\hbar^{2}}{2 m}\left[\left(\alpha+2 x_{0} \beta\right)^{2}+2 \beta^{2} w^{2}+\frac{1}{2} w^{-2}\right]+\frac{1}{2} m \omega^{2}\left(x_{0}^{2}+\frac{w^{2}}{2}\right)+\frac{\frac{1}{2} U_{0}|A|^{2}}{\sqrt{2}}\right\}
\end{aligned}
$$

Agora vamos às equações de movimento. Como estamos interessados somente no modo dipolar, vamos obter somente as equações necessárias à sua dedução:

$$
\begin{aligned}
& x_{0} \\
& \frac{\partial L}{\partial x_{0}}=\frac{d}{d t}\left(\frac{\partial L}{\partial \dot{x}_{0}}\right)=0 \\
& \frac{\partial}{\partial x_{0}}\left\{\frac{i}{2} \hbar\left(A \dot{A}^{*}-A^{*} \dot{A}\right) w \sqrt{\pi}+|A|^{2} w \sqrt{\pi}\left\{\hbar\left(\dot{\alpha} x_{0}+\dot{\beta} x_{0}^{2}+\dot{\beta} \frac{w^{2}}{2}\right)+\frac{\hbar^{2}}{2 m}\left[\left(\alpha+2 x_{0} \beta\right)^{2}+2 \beta^{2} w^{2}+\frac{1}{2} w^{-2}\right]+\frac{1}{2} m \omega^{2}\left(x_{0}^{2}+\frac{w^{2}}{2}\right)+\frac{\frac{1}{2} U_{0}|A|^{2}}{\sqrt{2}}\right\}\right\}= \\
& =\frac{d}{d t} \frac{\partial}{\partial \dot{x}_{0}}\left\{\frac{i}{2} \hbar\left(A \dot{A}^{*}-A^{*} \dot{A}\right) w \sqrt{\pi}+|A|^{2} w \sqrt{\pi}\left\{\hbar\left(\dot{\alpha} x_{0}+\dot{\beta} x_{0}^{2}+\dot{\beta} \frac{w^{2}}{2}\right)+\frac{\hbar^{2}}{2 m}\left[\left(\alpha+2 x_{0} \beta\right)^{2}+2 \beta^{2} w^{2}+\frac{1}{2} w^{-2}\right]+\frac{1}{2} m \omega^{2}\left(x_{0}^{2}+\frac{w^{2}}{2}\right)+\frac{\frac{1}{2} U_{0}|A|^{2}}{\sqrt{2}}\right\}\right\}=0 \\
& \frac{\partial}{\partial x_{0}}\left\{\frac{i}{2} \hbar\left(A \dot{A}^{*}-A^{*} \dot{A}\right) w \sqrt{\pi}+|A|^{2} w \sqrt{\pi}\left\{\hbar\left(\dot{\alpha} x_{0}+\dot{\beta} x_{0}^{2}+\dot{\beta} \frac{w^{2}}{2}\right)+\frac{\hbar^{2}}{2 m}\left[\left(\alpha+2 x_{0} \beta\right)^{2}+2 \beta^{2} w^{2}+\frac{1}{2} w^{-2}\right]+\frac{1}{2} m \omega^{2}\left(x_{0}^{2}+\frac{w^{2}}{2}\right)+\frac{\frac{1}{2} U_{0}|A|^{2}}{\sqrt{2}}\right\}\right\}=0 \\
& |A|^{2} \hbar w \sqrt{\pi}\left(\dot{\alpha}+2 \dot{\beta} x_{0}\right)+|A|^{2} w \sqrt{\pi} \frac{2 \hbar^{2}}{m}\left[\beta\left(\alpha+2 x_{0} \beta\right)\right]+|A|^{2} w \sqrt{\pi} m \omega^{2} x_{0}=0 \\
& \hbar\left(\dot{\alpha}+2 \dot{\beta} x_{0}\right)+m \omega^{2} x_{0}+\frac{2 \hbar^{2}}{m} \beta\left(\alpha+2 x_{0} \beta\right)=0 \\
& \text { (a) } \\
& \frac{\partial L}{\partial \alpha}=\frac{d}{d t}\left(\frac{\partial L}{\partial \dot{\alpha}}\right)
\end{aligned}
$$




$$
\begin{aligned}
& \frac{\partial}{\partial \alpha}\left\{\frac{i}{2} \hbar\left(A \dot{A}^{*}-A^{*} \dot{A}\right) w \sqrt{\pi}+|A|^{2} w \sqrt{\pi}\left\{\hbar\left(\dot{\alpha} x_{0}+\dot{\beta} x_{0}^{2}+\dot{\beta} \frac{w^{2}}{2}\right)+\frac{\hbar^{2}}{2 m}\left[\left(\alpha+2 x_{0} \beta\right)^{2}+2 \beta^{2} w^{2}+\frac{1}{2} w^{-2}\right]+\frac{1}{2} m \omega^{2}\left(x_{0}^{2}+\frac{w^{2}}{2}\right)+\frac{\frac{1}{2} U_{0}|A|^{2}}{\sqrt{2}}\right\}\right\}= \\
= & \frac{d}{d t} \frac{\partial}{\partial \dot{\alpha}}\left\{\frac{i}{2} \hbar\left(A \dot{A}^{*}-A^{*} \dot{A}\right) w \sqrt{\pi}+|A|^{2} w \sqrt{\pi}\left\{\hbar\left(\dot{\alpha} x_{0}+\dot{\beta} x_{0}^{2}+\dot{\beta} \frac{w^{2}}{2}\right)+\frac{\hbar^{2}}{2 m}\left[\left(\alpha+2 x_{0} \beta\right)^{2}+2 \beta^{2} w^{2}+\frac{1}{2} w^{-2}\right]+\frac{1}{2} m \omega^{2}\left(x_{0}^{2}+\frac{w^{2}}{2}\right)+\frac{\frac{1}{2} U_{0}|A|^{2}}{\sqrt{2}}\right\}\right\}
\end{aligned}
$$

$$
|A|^{2} w \sqrt{\pi}\left[\frac{\hbar^{2}}{m}\left(\alpha+2 x_{0} \beta\right)\right]=\frac{d}{d t}\left(|A|^{2} w \sqrt{\pi} \hbar x_{0}\right)
$$

$$
|A|^{2} w \frac{\hbar}{m}\left(\alpha+2 x_{0} \beta\right)=\frac{d}{d t}\left(|A|^{2} w x_{0}\right)=\left(\dot{A} A^{*}+A \dot{A}^{*}\right) w x_{0}+|A|^{2} \dot{w} x_{0}+|A|^{2} w \dot{x}_{0}
$$

$$
\frac{\hbar}{m}\left(\alpha+2 x_{0} \beta\right)=\left(\frac{\dot{A}}{A}+\frac{\dot{A}^{*}}{A^{*}}\right) x_{0}+\frac{\dot{w}}{w} x_{0}+\dot{x}_{0}
$$

Vamos para o modo dipolar a partir das duas equações obtidas. Neste modo, apenas $x_{0}$ muda com o tempo, isto é, $\dot{A}=\dot{A}^{*}=\dot{\alpha}=\dot{\beta}=\dot{w}=0$ :

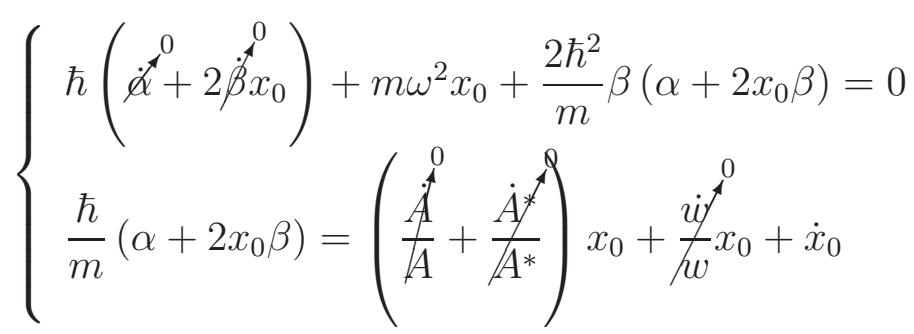

$$
\begin{aligned}
& \left\{\begin{array}{l}
m \omega^{2} x_{0}=-\frac{2 \hbar^{2} \beta}{m}\left(\alpha+2 x_{0} \beta\right) \\
\frac{\hbar}{m}\left(\alpha+2 x_{0} \beta\right)=\dot{x}_{0}
\end{array}\right.
\end{aligned}
$$

Derivando a segunda equação no tempo, temos:

$$
\begin{gathered}
\ddot{x}_{0}=\frac{\hbar}{m}\left(\ddot{\alpha}^{0}+2 \dot{x}_{0} \beta+2 x_{0} \ddot{\beta}\right)=\frac{2 \hbar \beta}{m} \dot{x}_{0}=\frac{2 \hbar \beta}{m}\left[\frac{\hbar}{m}\left(\alpha+2 x_{0} \beta\right)\right] \\
m \ddot{x}_{0}=\frac{2 \hbar^{2} \beta}{m}\left(\alpha+2 x_{0} \beta\right)=-m \omega^{2} x_{0}
\end{gathered}
$$

$$
\ddot{x}_{0}+\omega^{2} x_{0}=0
$$


Ou seja, temos a equação do modo em que o centro de massa do condensado se move conforme a equação do oscilador harmônico de frequência igual à frequência da armadilha, isto é:

$$
x_{0}(t)=A \cos (\omega t+\varphi)
$$

Dado que a posição inicial do centro de massa é $x_{C M}$ e a velocidade inicial é nula, temos:

$$
\left\{\begin{array}{l}
x_{0}(0)=A \cos (\varphi)=x_{C M} \\
\dot{x}_{0}(0)=-A \omega \operatorname{sen}(\varphi)=0
\end{array} \Rightarrow x_{0}(t)=x_{C M} \cos (\omega t)\right. \text { c.q.d. }
$$




\section{Dedução da equação do sóliton}

Vamos utilizar o seguinte ansatz, que descreve uma entidade de forma constante $(\varphi)$, que se desloca no tempo (exponencial temporal):

$$
\psi(x, t)=\sqrt{n} \varphi \mathrm{e}^{-i \mu t}
$$

onde $\varphi=\varphi\left(\zeta=\frac{x-v t}{\xi}\right), v$ corresponde à sua velocidade e $\xi$ é o healing lenght, que está relacionado à largura do sóliton. Substituindo esse ansatz na GPE sem o termo do potencial:

$$
\begin{gathered}
-\frac{1}{2} \frac{\partial^{2}}{\partial x^{2}}\left(\sqrt{n} \varphi \mathrm{e}^{-i \mu t}\right)+G_{0}\left|\sqrt{n} \varphi \mathrm{e}^{-i \mu t}\right|^{2} \sqrt{n} \varphi \mathrm{e}^{-i \mu t}=i \frac{\partial}{\partial t}\left(\sqrt{n} \varphi \mathrm{e}^{-i \mu t}\right) \\
-\frac{1}{2} \frac{\partial^{2}}{\partial x^{2}}\left(\varphi \mathrm{e}^{-i \mu t}\right)+n G_{0}|\varphi|^{2} \varphi \mathrm{e}^{-i \mu t}=i \frac{\partial}{\partial t}\left(\varphi \mathrm{e}^{-i \mu t}\right) \\
-\frac{1}{2} e^{-i n \hbar t} \frac{\partial^{2} \varphi}{\partial x^{2}}+n G_{0} e^{-i n t}|\varphi|^{2} \varphi=i\left(e^{-i n t} \frac{\partial \varphi}{\partial t}-i \mu \varphi e^{-i n t}\right) \\
-\frac{1}{2} \frac{\partial}{\partial \zeta}\left(\frac{d \varphi}{d \zeta} \frac{\partial \zeta}{\partial x}\right) \frac{\partial \zeta}{\partial x}+n G_{0}|\varphi|^{2} \varphi=i \frac{d \varphi}{d \zeta} \frac{\partial \zeta}{\partial t}+\mu \varphi \\
-\frac{1}{2} \frac{d}{d \zeta}\left(\frac{d \varphi}{d \zeta} \frac{1}{\xi}\right) \frac{1}{\xi}+n G_{0}|\varphi|^{2} \varphi=i \frac{d \varphi}{d \zeta}\left(-\frac{v}{\xi}\right)+\mu \varphi \\
-\frac{1}{2 \xi^{2}} \frac{d^{2} \varphi}{d \zeta^{2}}+n G_{0}|\varphi|^{2} \varphi=-i \frac{v}{\xi} \frac{d \varphi}{d \zeta}+\mu \varphi
\end{gathered}
$$

Tomando $\varphi=\varphi_{R}+i \varphi_{I}$ :

$$
-\frac{1}{2 \xi^{2}} \frac{d^{2}}{d \zeta^{2}}\left(\varphi_{R}+i \varphi_{I}\right)+n G_{0}\left|\varphi_{R}+i \varphi_{I}\right|^{2}\left(\varphi_{R}+i \varphi_{I}\right)=-i \frac{v}{\xi} \frac{d}{d \zeta}\left(\varphi_{R}+i \varphi_{I}\right)+\mu\left(\varphi_{R}+i \varphi_{I}\right)
$$




$$
\begin{gathered}
-\frac{1}{2 \xi^{2}} \frac{d^{2}}{d \zeta^{2}}\left(\varphi_{R}+i \varphi_{I}\right)+n G_{0}\left(\varphi_{R}^{2}+\varphi_{I}^{2}\right)\left(\varphi_{R}+i \varphi_{I}\right)=-i \frac{v}{\xi} \frac{d}{d \zeta}\left(\varphi_{R}+i \varphi_{I}\right)+\mu\left(\varphi_{R}+i \varphi_{I}\right) \\
\left\{\begin{array}{l}
-\frac{1}{2 \xi^{2}} \frac{d^{2} \varphi_{R}}{d \zeta^{2}}+n G_{0}\left(\varphi_{R}^{2}+\varphi_{I}^{2}\right) \varphi_{R}=\frac{v}{\xi} \frac{d \varphi_{I}}{d \zeta}+\mu \varphi_{R} \\
-\frac{1}{2 \xi^{2}} \frac{d^{2} \varphi_{I}}{d \zeta^{2}}+n G_{0}\left(\varphi_{R}^{2}+\varphi_{I}^{2}\right) \varphi_{I}=-\frac{v}{\xi} \frac{d \varphi_{R}}{d \zeta}+\mu \varphi_{I}
\end{array}\right.
\end{gathered}
$$

Tomando, sem perda de generalidade, a parte imaginária como constante no espaço:

$$
\left\{\begin{array}{l}
-\frac{1}{2 \xi^{2}} \frac{d^{2} \varphi_{R}}{d \zeta^{2}}+n G_{0}\left(\varphi_{R}^{2}+\varphi_{I}^{2}\right) \varphi_{R}=\mu \varphi_{R} \\
n G_{0}\left(\varphi_{R}^{2}+\varphi_{I}^{2}\right) \varphi_{I}=-\frac{v}{\xi} \frac{d \varphi_{R}}{d \zeta}+\mu \varphi_{I}
\end{array}\right.
$$

Da segunda equação:

$$
\begin{gathered}
\frac{v}{\xi} \frac{d \varphi_{R}}{d \zeta}=n G_{0}\left(\frac{\mu}{n G_{0}}-\varphi_{R}^{2}-\varphi_{I}^{2}\right) \varphi_{I} \\
\frac{v}{\xi} \int \frac{1}{\frac{\mu}{n G_{0}}-\varphi_{I}^{2}-\varphi_{R}^{2}} d \varphi_{R}=n G_{0} \varphi_{I} \int d \zeta
\end{gathered}
$$

Fazendo $\varphi_{R}=\sqrt{\frac{\mu}{n G_{0}}-\varphi_{I}^{2}} \operatorname{tgh}(\theta) \Rightarrow d \varphi_{R}=\sqrt{\frac{\mu}{n G_{0}}-\varphi_{I}^{2}} \operatorname{sech}^{2}(\theta) d \theta$ :

$$
\begin{gathered}
\frac{v}{\xi} \int \frac{\sqrt{\frac{\mu}{n G_{0}}-\varphi_{I}^{2}} \operatorname{sech}^{2}(\theta)}{\left(\frac{\mu}{n G_{0}}-\varphi_{I}^{2}\right) \operatorname{sech}^{2}(\theta)} d \theta=n G_{0} \varphi_{I} \zeta \\
\frac{v}{\xi \sqrt{\frac{\mu}{n G_{0}}-\varphi_{I}^{2}}} \theta=n G_{0} \varphi_{I} \zeta \\
\operatorname{tgh}(\theta)=\operatorname{tgh}\left(\frac{\xi \sqrt{\frac{\mu}{n G_{0}}-\varphi_{I}^{2}}}{v} n G_{0} \varphi_{I} \zeta\right)
\end{gathered}
$$




$$
\begin{gathered}
\frac{\varphi_{R}}{\sqrt{\frac{\mu}{n G_{0}}-\varphi_{I}^{2}}}=\operatorname{tgh}\left(\frac{\xi \sqrt{\frac{\mu}{n G_{0}}-\varphi_{I}^{2}}}{v} n G_{0} \varphi_{I} \zeta\right) \\
\varphi_{R}=\sqrt{\frac{\mu}{n G_{0}}-\varphi_{I}^{2}} \operatorname{tgh}\left(\frac{\xi \sqrt{\frac{\mu}{n G_{0}}-\varphi_{I}^{2}}}{v} n G_{0} \varphi_{I} \zeta\right)
\end{gathered}
$$

Substituindo, $\varphi_{R}$ na equação da parte real obtida acima:

$$
\begin{aligned}
& -\frac{1}{2 \xi^{2}} \frac{d^{2} \varphi_{R}}{d \zeta^{2}}+n G_{0}\left(\varphi_{R}^{2}+\varphi_{I}^{2}\right) \varphi_{R}=\mu \varphi_{R}
\end{aligned}
$$

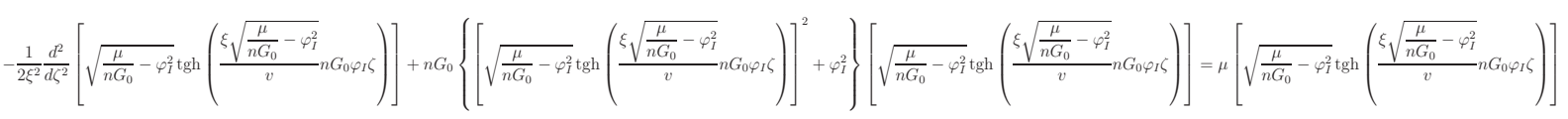

$$
\begin{aligned}
& -\frac{1}{2 \xi^{2}} d d^{2}\left[\operatorname{tgh}\left(\frac{\xi \sqrt{\frac{\mu}{n G_{0}}-\varphi_{1}^{2}}}{v} n G_{0} \varphi_{I} \zeta\right)\right]+n G_{0}\left\{\left[\sqrt{\frac{\mu}{n G_{0}}-\varphi_{I}^{2}} \operatorname{tgh}\left(\frac{\xi \sqrt{\frac{\mu}{n G_{0}}-\varphi_{I}^{2}}}{v} n G_{0} \varphi_{I} \zeta\right]^{2}+\varphi_{I}^{2}\right\} \operatorname{tgh}\left(\frac{\xi \sqrt{\frac{\mu}{n G_{0}}-\varphi_{I}^{2}}}{v} n G_{0} \varphi_{I} \zeta\right)=\mu \operatorname{tgh}\left(\frac{\xi \sqrt{\frac{\mu}{n G_{0}}-\varphi_{I}^{2}}}{v} n G_{0} \varphi_{1 \zeta} \zeta\right.\right.
\end{aligned}
$$

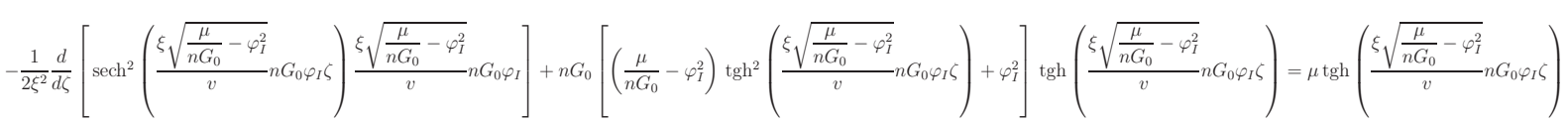

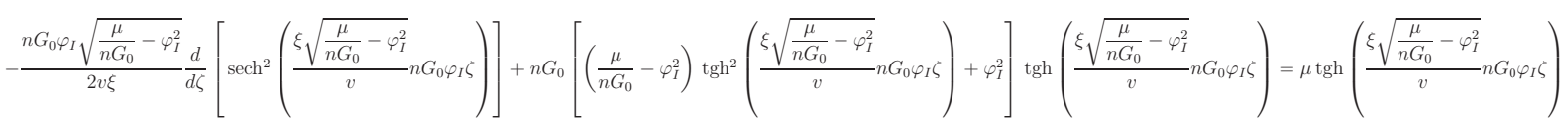

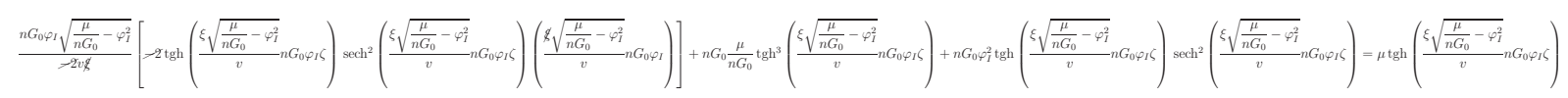

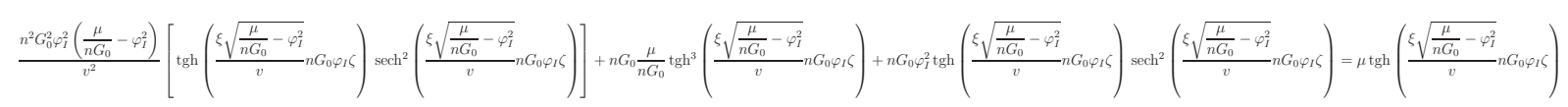




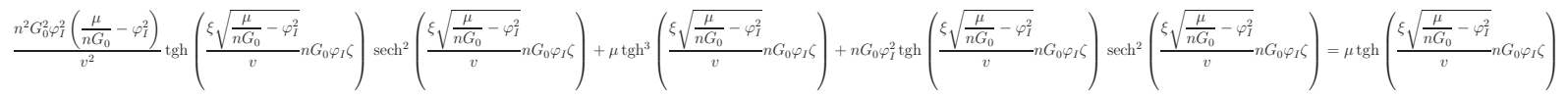

$$
\begin{aligned}
& {\left[\frac{n^{2} G_{0}^{2} \varphi_{1}^{2}\left(\frac{\mu}{n G_{0}}-\varphi_{1}^{2}\right)}{v^{2}}+n G_{0} \varphi_{1}^{2}\right] \operatorname{tgh}\left(\frac{\xi \sqrt{\frac{\mu}{n G_{0}}-\varphi_{1}^{2}}}{v} n G_{0} \varphi_{I} \zeta\right) \operatorname{sech}^{2}\left(\frac{\xi \sqrt{\frac{\mu}{n G_{0}}-\varphi_{1}^{2}}}{v} n G_{0} \varphi_{I} \zeta\right)=\mu \operatorname{tgh}\left(\frac{\xi \sqrt{\frac{\mu}{n G_{0}}-\varphi_{1}^{2}}}{v} n G_{0} \varphi_{Y \zeta}\right)\left[1-\operatorname{tgh}^{2}\left(\frac{\xi \sqrt{\frac{\mu}{n G_{0}}-\varphi_{1}^{2}}}{v} n G_{0} \varphi_{I} \zeta\right)\right]} \\
& {\left[\frac{n^{2} G_{0}^{2} \varphi_{I}^{2}\left(\frac{\mu}{n G_{0}}-\varphi_{1}^{2}\right)}{v^{2}}+n G_{0} \varphi_{I}^{2}\right] \operatorname{tgh}\left(\frac{\xi \sqrt{\frac{\mu}{n G_{0}}-\varphi_{I}^{2}}}{v} n G_{0} \varphi \zeta \zeta\right) \operatorname{sech}^{2}\left(\frac{\xi \sqrt{\frac{\mu}{n G_{0}}-\varphi_{I}^{2}}}{v} n G_{0} \varphi_{I} \zeta\right)=\mu \operatorname{tgh}\left(\frac{\xi \sqrt{\frac{\mu}{n G_{0}}-\varphi_{I}^{2}}}{v} n G_{0} \varphi \varphi_{1} \zeta\right) \operatorname{sech}^{2}\left(\frac{\xi \sqrt{\frac{\mu}{n G_{0}}-\varphi_{I}^{2}}}{v} n G_{0} \varphi \varphi_{I} \zeta\right)} \\
& \frac{n^{2} G_{0}^{2} \varphi_{I}^{2}\left(\frac{\mu}{n G_{0}}-\varphi_{I}^{2}\right)}{v^{2}}+n G_{0} \varphi_{I}^{2}=\mu \\
& \frac{n G_{0} \mu \varphi_{I}^{2}}{v^{2}}-\frac{n^{2} G_{0}^{2} \varphi_{I}^{4}}{v^{2}}+n G_{0} \varphi_{I}^{2}=\mu \\
& \left(n G_{0} \varphi_{I}^{2}\right)^{2}-\left(\mu+v^{2}\right)\left(n G_{0} \varphi_{I}^{2}\right)+v^{2} \mu=0
\end{aligned}
$$

Por soma e produto:

$$
\varphi_{I}^{2}=\left\{\begin{array}{l}
\frac{\mu /}{2 n G_{0}} \text { (Função constante e independente da velocidade do sóliton) } \\
\frac{v^{2}}{n G_{0}}
\end{array}\right.
$$

Tomando $\mu=\frac{1}{\xi^{2}}=n G_{0}=c^{2}$. 


$$
\begin{aligned}
\varphi_{R} & =\sqrt{\frac{\mu}{n G_{0}}-\frac{v^{2}}{n G_{0}}} \operatorname{tgh}\left(\frac{\xi \sqrt{\frac{\mu}{n G_{0}}-\frac{v^{2}}{n G_{0}}}}{v} n G_{0} \sqrt{\frac{v^{2}}{n G_{0}}} \zeta\right) \\
& =\sqrt{1-\frac{v^{2}}{c^{2}}} \operatorname{tgh}\left(\frac{\xi \sqrt{1-\frac{v^{2}}{c^{2}}}}{\not \varnothing} G_{0} \sqrt{\frac{\not \not^{2}}{n G_{0}}} \zeta\right) \\
& =\sqrt{1-\frac{v^{2}}{c^{2}}} \operatorname{tgh}\left(\sqrt{1-\frac{v^{2}}{c^{2}}} \zeta\right) \\
& =\sqrt{1-\frac{v^{2}}{c^{2}}} \operatorname{tgh}\left(\frac{x-v t}{\xi} \sqrt{1-\frac{v^{2}}{c^{2}}}\right)
\end{aligned}
$$

Voltando para $\varphi$ :

$$
\begin{aligned}
\varphi & =\varphi_{R}+i \varphi_{I} \\
& =\sqrt{1-\frac{v^{2}}{c^{2}}} \operatorname{tgh}\left(\frac{x-v t}{\xi} \sqrt{1-\frac{v^{2}}{c^{2}}}\right)+i \sqrt{\frac{v^{2}}{n G_{0}}} \\
& =\sqrt{1-\frac{v^{2}}{c^{2}}} \operatorname{tgh}\left(\frac{x-v t}{\xi} \sqrt{1-\frac{v^{2}}{c^{2}}}\right)+i \frac{v}{c}
\end{aligned}
$$

Portanto:

$$
\psi=\sqrt{n} \varphi \mathrm{e}^{-i c^{2} t}=\sqrt{n}\left[\sqrt{1-u^{2}} \operatorname{tgh}\left(\frac{x-v t}{\xi} \sqrt{1-u^{2}}\right)+i u\right] \mathrm{e}^{-i c^{2} t}
$$

com $u=\frac{v}{c}$, sendo $v(0 \leqslant v \leqslant c)$ a velocidade do sóliton e $c$ a velocidade do som no meio estudado, $x_{0}=v t$ a posição do core do sóliton e $\xi$ o chamado healing length, que é proporcional à largura do sóliton. 


\section{Dedução da energia do sóliton}

Vamos calcular a energia do sóliton em BEC na ausência de potencial externo através da diferença entre os funcionais de energia do sistema com e sem sóliton:

$$
\begin{aligned}
& E_{\text {soliton }}=\int_{-\infty}^{\infty} \frac{1}{2}\left|\frac{d \psi_{0}}{d x}\right|^{2}+\frac{G_{0}}{2}\left(\left|\psi_{0}\right|^{2}-n\right)^{2} d x \\
& E=\int_{-\infty}^{\infty} \frac{1}{2} \frac{\left(1-u^{2}\right)^{2}}{\xi^{2}} n \operatorname{sech}^{4}\left(\frac{x-x_{0}}{\xi} \sqrt{1-u^{2}}\right)+\frac{G_{0}}{2}\left[n\left(1-u^{2}\right) \operatorname{tgh}^{2}\left(\frac{x-x_{0}}{\xi} \sqrt{1-u^{2}}\right)-n\left(1-u^{2}\right)\right]^{2} d x \\
& E=\int_{-\infty}^{\infty} n \frac{1}{2} \frac{\left(1-u^{2}\right)^{2}}{\xi^{2}} \operatorname{sech}^{4}\left(\frac{x-x_{0}}{\xi} \sqrt{1-u^{2}}\right)+n^{2} \frac{G_{0}}{2}\left[\left(1-u^{2}\right) \operatorname{sech}^{2}\left(\frac{x-x_{0}}{\xi} \sqrt{1-u^{2}}\right)\right]^{2} d x \\
& E=n\left(\frac{1}{2 \xi^{2}}+\frac{n G_{0}}{2}\right)\left(1-u^{2}\right)^{2} \int_{-\infty}^{\infty} \operatorname{sech}^{4}\left(\frac{x-x_{0}}{\xi} \sqrt{1-u^{2}}\right) d x \\
& E=n\left(\frac{1}{2 \xi^{2}}+\frac{n G_{0}}{2}\right)\left(1-u^{2}\right)^{2}\left(\frac{4}{3 \frac{\sqrt{1-u^{2}}}{\xi}}\right)=n\left(\frac{1}{2}+\frac{1}{2}\right) c^{2}\left(1-u^{2}\right)^{3 / 2}\left(\frac{4}{3 c}\right) \\
& E_{\text {soliton }}=\frac{4}{3} n c\left(1-u^{2}\right)^{3 / 2}
\end{aligned}
$$




\section{Cálculo do potencial químico do BEC na presença da barreira através da TFA}

Como já foi descrito anteriormente, temos, no caso geral da aproximação de TF unidimensional, as seguintes equações:

$$
\begin{aligned}
& n(x)=|\psi(x)|^{2}=\frac{\mu-V(x)}{G_{0}} \\
& N=\int_{|x|<R_{T F}} n(x) d x
\end{aligned}
$$

E nesse caso temos o seguinte potencial:

$$
V(x)=\frac{1}{2} \omega^{2} x^{2}+h_{\text {barr }} \mu \max \left\{0 ; 1-\left(\frac{x}{r_{b a r r} R_{T F}}\right)^{2}\right\}
$$

Vamos então começar pela densidade:

$$
\begin{gathered}
n(x)=|\psi(x)|^{2}=\frac{\mu-V(x)}{G_{0}} \\
n(x)=\frac{1}{G_{0}}\left\{\begin{array}{l}
\mu-\frac{1}{2} \omega^{2} x^{2} \\
\mu-\frac{1}{2} \omega^{2} x^{2}-h_{\text {barr }} \mu\left[1-\left(\frac{x}{r_{\text {barr }} R_{T F}}\right)^{2}\right] \quad \begin{array}{l}
r_{\text {barr }} R_{T F}<|x|<R_{T F}=\frac{\sqrt{2 \mu}}{\omega} \\
|x|<r_{\text {barr }} R_{T F}
\end{array}
\end{array}\right.
\end{gathered}
$$

Agora através da função densidade podemos obter $\mu$ : 


$$
N=\int_{|x|<R_{T F}} n(x) d x
$$

Vamos considerar a função de onda normalizada $(N=1)$ :

$$
\frac{1}{G_{0}} \int_{|x|<r_{\text {barr }} R_{T F}} \mu-\frac{1}{2} \omega^{2} x^{2}-h_{\text {barr }} \mu\left[1-\left(\frac{x}{r_{\text {barr }} R_{T F}}\right)^{2}\right] d x+\frac{1}{G_{0}} \int_{r_{\text {barr }} R_{T F}<|x|<R_{T F}} \mu-\frac{1}{2} \omega^{2} x^{2} d x=1
$$

$$
\begin{gathered}
\int_{-R_{T F}}^{R_{T F}} \mu-\frac{1}{2} \omega^{2} x^{2} d x-h_{\text {barr }} \mu \int_{-r_{\text {barr }} R_{T F}}^{r_{\text {barr }} R_{T F}} 1-\left(\frac{x}{r_{\text {barr }} R_{T F}}\right)^{2} d x=G_{0} \\
{\left[\mu x-\frac{1}{6} \omega^{2} x^{3}\right]_{-R_{T F}}^{R_{T F}}-h_{\text {barr }} \mu\left[x-\frac{x^{3}}{3\left(r_{\text {barr }} R_{T F}\right)^{2}}\right]_{-r_{\text {barr }} R_{T F}}^{r_{b a r r} R_{T F}}=G_{0}} \\
{\left[\mu R_{T F}-\frac{1}{6} \omega^{2} R_{T F}^{3}\right]-h_{\text {barr }} \mu\left[r_{\text {barr }} R_{T F}-\frac{\left(r_{b a r r} R_{T F}\right)^{\not p}}{3\left(r_{b a r r} R_{T F}\right)^{2}}\right]=\frac{G_{0}}{2}} \\
\mu R_{T F}-\frac{1}{6} \omega^{2} R_{T F}^{3}-\frac{2}{3} h_{\text {barr }} \mu r_{b a r r} R_{T F}=\frac{G_{0}}{2}
\end{gathered}
$$

Fazendo $\mu=\frac{1}{2} \omega^{2} R_{T F}^{2}$ :

$$
\begin{gathered}
2 R_{T F}^{3}-2 h_{b a r r} r_{b a r r} R_{T F}^{3}=\frac{3 G_{0}}{\omega^{2}} \\
R_{T F}^{3}=\frac{3}{2} \frac{G_{0}}{\omega^{2}\left(1-h_{\text {barr }} r_{b a r r}\right)}
\end{gathered}
$$

Voltando agora para $\mu$ fazendo $R_{T F}=\frac{\sqrt{2 \mu}}{\omega}$ :

$$
\begin{gathered}
\left(\frac{\sqrt{2 \mu}}{\omega}\right)^{3}=\frac{3}{2} \frac{G_{0}}{\omega^{2}\left(1-h_{\text {barr }} r_{\text {barr }}\right)} \\
\mu=\frac{1}{2}\left(\frac{3}{2} \frac{\omega G_{0}}{1-h_{\text {barr }} r_{\text {barr }}}\right)^{2 / 3}
\end{gathered}
$$

